\title{
Vascular cognitive impairment and cholinergic function : an integrative analysis in rat brain
}

Citation for published version (APA):

Hoff, E. (2009). Vascular cognitive impairment and cholinergic function : an integrative analysis in rat brain. [Doctoral Thesis, Maastricht University]. [Maastricht University].

https://doi.org/10.26481/dis.20090326eh

Document status and date:

Published: 01/01/2009

DOI:

10.26481/dis.20090326eh

Document Version:

Publisher's PDF, also known as Version of record

\section{Please check the document version of this publication:}

- A submitted manuscript is the version of the article upon submission and before peer-review. There can be important differences between the submitted version and the official published version of record.

People interested in the research are advised to contact the author for the final version of the publication, or visit the DOI to the publisher's website.

- The final author version and the galley proof are versions of the publication after peer review.

- The final published version features the final layout of the paper including the volume, issue and page numbers.

Link to publication

\footnotetext{
General rights rights.

- You may freely distribute the URL identifying the publication in the public portal. please follow below link for the End User Agreement:

www.umlib.nl/taverne-license

Take down policy

If you believe that this document breaches copyright please contact us at:

repository@maastrichtuniversity.nl

providing details and we will investigate your claim.
}

Copyright and moral rights for the publications made accessible in the public portal are retained by the authors and/or other copyright owners and it is a condition of accessing publications that users recognise and abide by the legal requirements associated with these

- Users may download and print one copy of any publication from the public portal for the purpose of private study or research.

- You may not further distribute the material or use it for any profit-making activity or commercial gain

If the publication is distributed under the terms of Article $25 \mathrm{fa}$ of the Dutch Copyright Act, indicated by the "Taverne" license above, 


\section{Vascular cognitive impairment and cholinergic function: an integrative analysis in rat brain}


ISBN/EAN 978-90-9024007-7

(๑) Copyright Erik I. Hoff, Maastricht 2009
Lay-out:
Danitsja M. Hoff-van Leeuwen; Erik I. Hoff
Omslagillustratie:
Remco J.J. Knol; Datawyse Boekproducties, Maastricht
Omslagrealisatie en druk: Datawyse Boekproducties, Maastricht

This thesis was prepared at (1) the School for Mental Health and Neuroscience of Maastricht University, which participates in the European Graduate School of Neuroscience (EURON) accredited by the Royal Dutch Academy of Arts and Sciences (KNAW), and (2) the Image Sciences Institute participating in the Research School for Biomedical Image Sciences (ImagO) and the Utrecht Graduate School of Life Sciences. 


\section{Vascular cognitive impairment and cholinergic function: an integrative analysis in rat brain}

\section{PROEFSCHRIFT}

ter verkrijging van de graad van doctor aan de Universiteit Maastricht, op gezag van de Rector Magnificus, Prof. mr. G.P.M.F. Mols,

volgens het besluit van het College van Decanen,

in het openbaar te verdedigen op

donderdag 26 maart 2009 om 16:00 uur

door

\section{Erik Inne Hoff}

geboren te Amsterdam op 17 januari 1975 


\section{Promotores}

Prof. dr. H.W.M. Steinbusch

Prof. dr. M. Limburg

\section{Copromotores}

Dr. R.J. van Oostenbrugge

Dr. R.M. Dijkhuizen, Universiteit Utrecht

\section{Beoordelingscommissie}

Prof. dr. J.S.H. Vles (voorzitter)

Prof. dr. K. Nicolay, Technische Universiteit Eindhoven

Dr. J. Prickaerts

Prof. dr. Ph. Scheltens, Vrije Universiteit Amsterdam

Prof. dr. F.R.J. Verhey 


\section{Contents}

$\begin{array}{lll}\text { Chapter } 1 \text { General introduction } & 9\end{array}$

Chapter 2 In vivo visualisation of vascular leakage in photochemically induced cortical infarction

Chapter 3 Dissociable effects in reaction time performance after unilateral cerebral infarction: a comparison between the left and right frontal cortices in rats

Chapter 4 Effects of right hemisphere cortical infarction and muscarinic acetylcholine receptor blockade on spatial visual attention performance in rats

Chapter 5 Pharmacological magnetic resonance imaging of muscarinic 69 acetylcholine receptor activation in rat brain

Chapter 6 Alterations in the cholinergic system after frontal cortex infarction in rat brain: pharmacological magnetic resonance imaging of muscarinic receptor activation and stereological analysis of cholinergic basal forebrain neurons

Chapter 7 General discussion

Appendix Pharmacological magnetic resonance imaging of muscarinic acetylcholine receptor activation after subcortical ischemia in rat brain

Summary

Samenvatting

Dankwoord

Curriculum vitae

Publications 


\section{ABbreviations}

\begin{tabular}{|c|c|}
\hline $\mathrm{AChR}$ & acetylcholine receptor \\
\hline mAChR & muscarinic $A C h R$ \\
\hline $\mathrm{nAChR}$ & nicotinic $A C h R$ \\
\hline$A D$ & Alzheimer's disease \\
\hline ANOVA & analysis of variance \\
\hline BOLD & blood oxygen level dependent \\
\hline CBV & cerebral blood flow \\
\hline ChAT & choline acetyl transferase \\
\hline $\mathrm{CO}_{2}$ & carbon dioxide \\
\hline ICA & independent component analysis \\
\hline FOV & field of view \\
\hline GIFT & Group ICA of fMRI Toolbox \\
\hline GLM & general linear model \\
\hline ITI & inter-trial interval \\
\hline MCAO & middle cerebral artery occlusion \\
\hline MR & magnetic resonance \\
\hline MRI & MR imaging \\
\hline fMRI & functional MRI \\
\hline phMRI & pharmacological fMRI \\
\hline MT & motor time \\
\hline $\mathrm{N}_{2} \mathrm{O}$ & nitrous oxide \\
\hline $\mathrm{O}_{2}$ & oxygen \\
\hline PR & premature responses \\
\hline ROI & region of interest \\
\hline RT & reaction time \\
\hline SPM & statistical parametric mapping \\
\hline SPSS & statistical package for the Social Sciences \\
\hline TBS & Tris-buffered saline \\
\hline TBS-T & TBS with Triton \\
\hline TE & echo time \\
\hline TR & relaxation time \\
\hline $\mathrm{VaD}$ & vascular dementia \\
\hline $\mathrm{VCl}$ & vascular cognitive impairment \\
\hline
\end{tabular}





\section{HORIZON}

Dichtbij is soms de horizon:

Mijn blikveld is benauwd en krap

De wereld is in ballingschap

Het hier in mij is hoofdstation.

Ver weg is soms de horizon:

De ruimte dat ik even snap

Mijn eigen medezeggenschap

De verte is mijn peilstation.

Soms na, soms ver de horizon:

Bepaal hem zelf bij elke stap,

Creëer mijn eigen meesterschap,

De einder is geen eindstation.

(Ineke Hoonakker) 


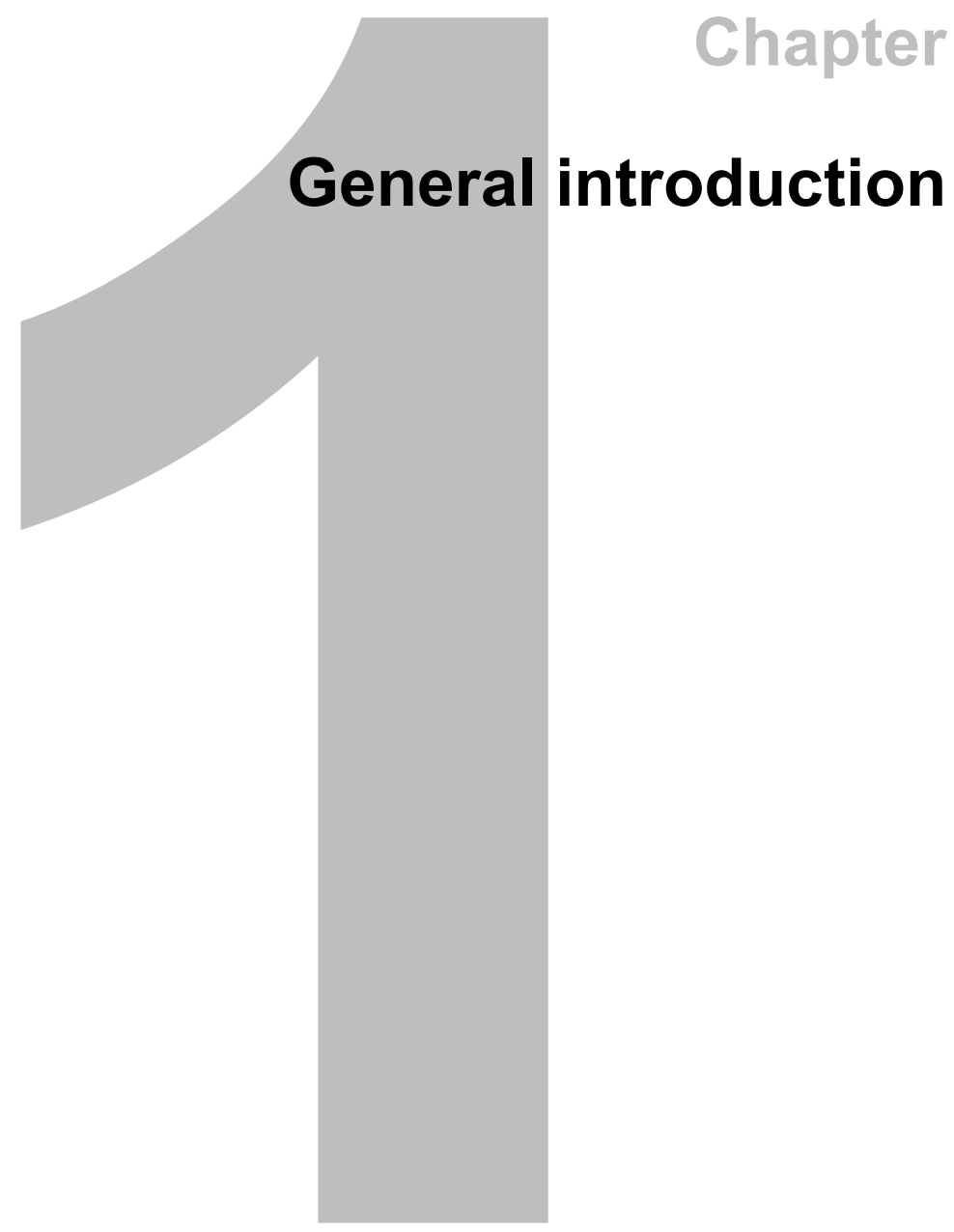


Stroke is the third leading cause of death and the most important cause of long-term disability in the adult population in the Western world. In the Netherlands, an estimated 40.000 people suffer a stroke each year and over 200.000 patients have to live with its consequences [1]. Stroke incidence is strongly age-dependent, more and more patients survive other types of vascular diseases such as coronary heart disease, and life expectancy is ever increasing. As a result, the number of stroke patients is expected to increase by $40-50 \%$ in the next 20 years. Besides the commonly recognized sensorimotor handicaps, stroke has a great impact on cognitive and emotional functioning of patients.

\section{Vascular cognitive impairment}

Impairment of cognitive function is common after stroke. Studies report the occurrence of cognitive impairment in at least one-half of stroke patients, ranging from mild cognitive impairment to dementia [2-7]. Physical handicaps rather than cognitive deficits generally attract most attention. However, post-stroke cognitive impairment has important consequences since it is associated with poorer prognosis and outcome. Vascular cognitive impairment $(\mathrm{VCl})$ has been proposed as an umbrella term to include subjects affected with any degree of cognitive impairment resulting from cerebrovascular disease, including vascular dementia (VaD) [8, 9]. There is considerable debate with respect to the specificity of cognitive profiles, anatomical correlates and pathological substrates associated with $\mathrm{VCl}$.

\section{Neuropsychological profile of vascular cognitive impairment}

The cognitive profile of $\mathrm{VCl}$ has been found to be predominantly of the subcortical type [10-13]. One of the more robust neuropsychological characteristics of patients with subcortical disease is psychomotor slowing and deficits in executive functions with relatively preserved language and recognition memory. Executive functions that tend to be disproportionately impaired include planning and sequencing, speed of mental processing, performance on unstructured tasks and attention. In $\mathrm{VCl}$, speed of information processing has consistently been identified as impaired whereas deficits in executive performance and attention are less pronounced [12, 14, 15]. Deficits in speed of information processing are found even in patients that do not meet criteria for vascular cognitive impairment $[16,17]$. 


\section{Neuroanatomical correlates of vascular cognitive impairment}

Whereas localized infarction of the cerebral cortex may cause specific disturbances of higher cortical functions, ischemic lesions of subcortical grey and white matter generally do not result in impairment of a particular cognitive domain. The cognitive expression of isolated white matter lesions appears to be limited to mild motor and psychomotor slowing rather than a pervasive, severe degree of cognitive impairment [18]. Epidemiological studies have established an association of periventricular white matter lesions, brain infarction and generalized atrophy with poorer performance in certain cognitive domains, particularly information processing speed and executive function [19-21]. On the basis of current neuroimaging and post-mortem data, three anatomical concepts have been described that may explain $\mathrm{VCl}$ [22]: (a) a critical volume of accumulated cortical infarcts, (b) strategically localized subcortical infarcts; and (c) functional disconnection of neuronal networks. Cortical neuronal loss as well as subcortical axonal injury might thus be key elements in the pathogenesis and progression of $\mathrm{VCl}$. Moreover, irreversible injury to brain parenchyma causes upstream and downstream effects in neuronal networks through mechanisms such as anterograde (Wallerian) degeneration, retrograde degeneration and apoptosis.

\section{Vascular cognitive impairment and cholinergic dysfunction}

There is abundant evidence that the basal forebrain cholinergic system plays a major role in cognitive performance. The central cholinergic system consists of a diffuse neuronal network of projections from several basal forebrain nuclei towards the cerebral cortex and hippocampus which exerts its function at synapses through the endogenous neurotransmitter acetylcholine. The knowledge on cholinergic function in cognition stems from psychopharmacological studies on the effects of cholinergic drugs in humans [23-25], as well as studies using pharmacological manipulations and selective lesioning of basal forebrain cholinergic neurons in animals [26-31]. Recent experiments suggest that cholinergic neurons serve a modulatory function by optimizing cortical information processing and influencing attention [32]. Based on these studies, a strong correlation between cognitive performance and cholinergic function has been established.

Vascular cognitive impairment associated with cholinergic dysfunction has mainly been investigated in vascular dementia $(\mathrm{VaD})$ and Alzheimer's disease (AD) [33]. Recent evidence shows that deficits in cholinergic neuronal markers and degeneration of neuronal circuits contribute to cognitive decline in both $A D$ and $\mathrm{VaD}$. This suggests that central cholinergic function is impaired in $\mathrm{VaD}$, which may underlie the 
cognitive and functional deficits characteristic of $\mathrm{VCl}$. Vascular cognitive impairment in $\mathrm{VaD}$ may arise directly from ischemic loss of cholinergic neurons in basal forebrain nuclei. Alternatively, loss of cognitive function may arise indirectly, following ischemic damage to cortical and subcortical cholinergic pathways.

\section{Experimental stroke research and cognitive impairment}

Ischemic stroke is a disease with a complex multifactorial pathophysiology. Animals models of focal cerebral ischemia are valuable tools to study the underlying mechanisms, and by that are widely used in the development of novel treatment regimens for ischemic cerebral injury [34-37]. Most of the experimental stroke models are carried out on rodents, and each model has its particular strengths and weaknesses. Focal cerebral ischemia can be induced by common carotid artery occlusion, middle cerebral artery occlusion, and microcirculatory occlusion [38]. Mimicking all aspects of human ischemic stroke in a single animal model is not possible, since ischemic stroke itself is a very heterogeneous disorder. The photothrombotic model described in this thesis produces coagulation of the cortical microcirculation. The permanent vascular occlusion resulting from endothelial damage and platelet aggregation resembles the pathophysiology of cerebral ischemia due to injury of a small vessel in humans [39]. The advantages of the model include its non-invasiveness (no craniectomy is required, merely retraction of the skin over the skull), high reproducibility (of lesion size and location), and short procedure duration (such that subsequent behavioural studies can be undertaken within hours). The development of tissue damage is very rapid and without reperfusion or collateral blood flow. In this respect, however, the model has relatively limited clinical relevance and has shown little positive results in studies testing possible neuroprotective agents [40].

In search of neuroprotective drugs in acute stroke, a vast amount of experimental stroke research has been aimed at preventing or ameliorating structural damage after an ischemic insult. This approach has thus far not yielded a standard pharmacological therapy of use in clinical neurological practice [38, 41, 42]. Traditionally, poststroke damage has been assessed structurally by comparing histological examination in animal models that did or did not receive a pharmacological treatment. Many stroke researchers have nowadays expanded their techniques to assess the effects of stroke functionally by using long-term behavioural correlates of histologicallydetermined brain infarction in animal models [43]. As compared to motor performance, however, cognitive performance is seldomly used as a functional behavioural endpoint in experimental stroke research. 
The vast majority of studies on behavioural outcomes of experimental stroke has been conducted in rodents. Various studies have shown impaired performance in behavioural tasks measuring cognitive processes such as attention, learning and memory [44-49]. However, most experimental stroke models typically produce extensive damage in both cortex and subcortex resulting in disturbances of sensorimotor abilities [50]. Alterations in motor function, sensory capabilities, or motivation can become confounding factors when measuring cognitive performance. Moreover, mnemonic functions are relatively preserved in VCl. Studying vascular cognitive impairment in an experimental setting may therefore require the use of a relatively restricted lesion model and behavioural tasks measuring cognitive functions other than learning and memory.

\section{Aim of the thesis}

The present thesis explores the putative relationship between the development of vascular cognitive impairment and damage to the central cholinergic system in an animal model of cerebral infarction. It is assumed that disruption of cholinergic pathways secondary to focal infarction of the cerebral cortex in the rat results in impaired cognitive performance.

(a) The first objective was to establish an equivalent of human vascular cognitive impairment in an animal model of focal cerebral infarction.

(b) The second objective was to characterize structural and functional changes to the central cholinergic system after focal cerebral cortical infarction.

\section{Outline of the thesis}

Vascular cognitive impairment in humans is thought to result from a combination of localized damage to both grey and white matter, as well as functional disconnection of neuronal networks by cerebral ischemia. There are numerous animal models to study experimental brain ischemia. The photothrombotic model is able to produce a relatively small focal infarction of the cerebral cortex, which allows for the investigation of (1) cognitive effects without confounding by gross sensorimotor deficits and (2) local as well as remote changes to cholinergic neurotransmission. We first performed an in vivo study to further clarify the pathophysiological processes that lead to brain infarction in this photothrombotic model. As such, chapter 2 describes the contribution of vascular leakage in the development of photothrombotic infarction. 
The first objective of this thesis was to establish an equivalent of human vascular cognitive impairment in an animal model of focal cerebral infarction. The neuropsychological profile of $\mathrm{VCl}$ is typically characterized by deficits in psychomotor processing speed and attention performance. Both types of deficits were studied separately. First, in chapter 3 , a reaction time task is used to detect whether information processing speed is slowed following unilateral photothrombotic infarction of the frontal cortices in rats. Second, chapter 4 studies whether performance is impaired in a behavioural paradigm measuring sustained attention performance after photothrombotic infarction of either the frontal or parietal cortex in rats. Also in this chapter, the involvement of the cholinergic system in cognitive performance is described by measuring whether sustained attention is reduced after pharmacological cholinergic muscarinic receptor blockade.

The second objective of this thesis was to characterize functional and structural changes to the central cholinergic system after focal cerebral cortical infarction. To this end, a method was sought that would enable whole-brain assessment of the cholinergic system in a living animal. Chapter 5 describes the feasibility of pharmacological functional magnetic resonance imaging (phMRI) as a means to detect cholinergic muscarinic receptor activation in rat brain. Subsequently, we tested the applicability of this neuroimaging method to measure cholinergic muscarinic receptor activation after subcortical brain infarction (appendix). This was done in order to investigate cholinergic function after direct ischemic damage of basal forebrain cholinergic neurons projecting toward the cerebral cortex. The reverse is described in chapter 6, where remote cholinergic muscarinic receptor activation as well as cholinergic cell number in the basal forebrain were studied after photothrombotic infarction to the frontal cortex. Finally, a general discussion is presented in chapter 7 . 


\section{REFERENCES}

1. Bots ML, van Dis SJ. Beroerte: cijfers en feiten: Hartstichting Nederland; 2006.

2. Tatemichi TK, Desmond DW, Stern Y, Paik M, Sano M, Bagiella E. Cognitive impairment after stroke: frequency, patterns, and relationship to functional abilities. J Neurol Neurosurg Psychiatry. 1994;57(2):202-7.

3. Hochstenbach J, Mulder T, van Limbeek J, Donders R, Schoonderwaldt H. Cognitive decline following stroke: a comprehensive study of cognitive decline following stroke. J Clin Exp Neuropsychol. 1998;20(4):503-17.

4. Tham W, Auchus AP, Thong M, Goh ML, Chang HM, Wong MC, et al. Progression of cognitive impairment after stroke: one year results from a longitudinal study of Singaporean stroke patients. J Neurol Sci. 2002 Nov 15;203-204:49-52.

5. Rasquin SM, Verhey FR, Lousberg R, Winkens I, Lodder J. Vascular cognitive disorders: memory, mental speed and cognitive flexibility after stroke. J Neurol Sci. 2002 Nov 15;203-204:115-9.

6. Mok VC, Wong A, Lam WW, Fan YH, Tang WK, Kwok T, et al. Cognitive impairment and functional outcome after stroke associated with small vessel disease. J Neurol Neurosurg Psychiatry. 2004 Apr;75(4):560-6.

7. Serrano S, Domingo J, Rodriguez-Garcia E, Castro MD, del Ser T. Frequency of cognitive impairment without dementia in patients with stroke: a two-year follow-up study. Stroke. 2007 Jan;38(1):105-10.

8. bowler JV, Hachinski V. Vascular Cognitive impairement: a new approach to vascular dementia. Vascular Dementia; 1995. p. 357-76.

9. Roman GC, Sachdev P, Royall DR, Bullock RA, Orgogozo JM, Lopez-Pousa S, et al. Vascular cognitive disorder: a new diagnostic category updating vascular cognitive impairment and vascular dementia. J Neurol Sci. 2004 Nov 15;226(1-2):81-7.

10. O'Brien JT, Erkinjuntti T, Reisberg B, Roman G, Sawada T, Pantoni L, et al. Vascular cognitive impairment. Lancet Neurol. 2003 Feb;2(2):89-98.

11. Graham NL, Emery T, Hodges JR. Distinctive cognitive profiles in Alzheimer's disease and subcortical vascular dementia. J Neurol Neurosurg Psychiatry. 2004 Jan;75(1):61-71.

12. Sachdev PS, Brodaty H, Valenzuela MJ, Lorentz L, Looi JC, Wen W, et al. The neuropsychological profile of vascular cognitive impairment in stroke and TIA patients. Neurology. 2004 Mar 23;62(6):912-9.

13. Desmond DW. The neuropsychology of vascular cognitive impairment: is there a specific cognitive deficit? J Neurol Sci. 2004 Nov 15;226(1-2):3-7.

14. de Groot JC, de Leeuw FE, Oudkerk M, van Gijn J, Hofman A, Jolles J, et al. Cerebral white matter lesions and cognitive function: the Rotterdam Scan Study [see comments]. Ann Neurol. 2000;47(2):14551.

15. Selnes OA, Vinters HV. Vascular cognitive impairment. Nat Clin Pract Neurol. 2006 Oct;2(10):538-47.

16. Stephens S, Kenny RA, Rowan E, Allan L, Kalaria RN, Bradbury M, et al. Neuropsychological characteristics of mild vascular cognitive impairment and dementia after stroke. Int J Geriatr Psychiatry. 2004 Nov;19(11):1053-7.

17. Rasquin SM. Vascular Cognitive Impairment: phenomenology, course, risk factors [doctoral thesis]. Maastricht: Maastricht University; 2004.

18. Mosley TH, Jr., Knopman DS, Catellier DJ, Bryan N, Hutchinson RG, Grothues CA, et al. Cerebral MRI findings and cognitive functioning: the Atherosclerosis Risk in Communities study. Neurology. 2005 Jun 28;64(12):2056-62.

19. Prins ND, van Dijk EJ, den Heijer T, Vermeer SE, Jolles J, Koudstaal PJ, et al. Cerebral small-vessel disease and decline in information processing speed, executive function and memory. Brain. 2005 Sep;128(Pt 9):2034-41. 
20. Roman GC, Erkinjuntti T, Wallin A, Pantoni L, Chui HC. Subcortical ischaemic vascular dementia. Lancet Neurol. 2002 Nov;1(7):426-36.

21. de la Torre JC. Is Alzheimer's disease a neurodegenerative or a vascular disorder? Data, dogma, and dialectics. Lancet Neurol. 2004 Mar;3(3):184-90.

22. Haring HP. Cognitive impairment after stroke. Curr Opin Neurol. 2002 Feb;15(1):79-84.

23. Furey ML, Pietrini P, Haxby JV, Alexander GE, Lee HC, VanMeter J, et al. Cholinergic stimulation alters performance and task-specific regional cerebral blood flow during working memory. Proc Natl Acad Sci U S A. 1997 Jun 10;94(12):6512-6.

24. Gitelman DR, Prohovnik I. Muscarinic and nicotinic contributions to cognitive function and cortical blood flow. Neurobiol Aging. 1992 Mar-Apr;13(2):313-8.

25. Sitaram N, Weingartner H, Gillin JC. Human serial learning: enhancement with arecholine and choline impairment with scopolamine. Science. 1978 Jul 21;201(4352):274-6.

26. Sarter M, Parikh V. Choline transporters, cholinergic transmission and cognition. Nat Rev Neurosci. 2005 Jan;6(1):48-56.

27. Everitt BJ, Robbins TW. Central cholinergic systems and cognition. Annu Rev Psychol. 1997;48:649-84.

28. Iversen SD. Behavioural evaluation of cholinergic drugs. Life Sci. 1997;60(13-14):1145-52.

29. Schliebs R, Rossner S, Bigl V. Immunolesion by 192lgG-saporin of rat basal forebrain cholinergic system: a useful tool to produce cortical cholinergic dysfunction. Prog Brain Res. 1996;109:253-64.

30. Decker MW. Animal models of cognitive function. Crit Rev Neurobiol. 1995;9(4):321-43.

31. Gage FH, Chen KS, Buzsaki G, Armstrong D. Experimental approaches to age-related cognitive impairments. Neurobiol Aging. 1988 Sep-Dec;9(5-6):645-55.

32. Baxter MG, Chiba AA. Cognitive functions of the basal forebrain. Curr Opin Neurobiol. 1999 Apr;9(2):178-83.

33. Roman GC, Kalaria RN. Vascular determinants of cholinergic deficits in Alzheimer disease and vascular dementia. Neurobiol Aging. 2006 Dec;27(12):1769-85.

34. Durukan A, Tatlisumak T. Acute ischemic stroke: overview of major experimental rodent models, pathophysiology, and therapy of focal cerebral ischemia. Pharmacol Biochem Behav. 2007 May;87(1):17997.

35. Hossmann KA. Cerebral ischemia: models, methods and outcomes. Neuropharmacology. 2008 Sep;55(3):257-70.

36. Fisher M, Henninger N. Translational research in stroke: taking advances in the pathophysiology and treatment of stroke from the experimental setting to clinical trials. Curr Neurol Neurosci Rep. 2007 Jan;7(1):35-41.

37. Alonso de Lecinana M, Diez-Tejedor E, Gutierrez M, Guerrero S, Carceller F, Roda JM. New goals in ischemic stroke therapy: the experimental approach--harmonizing science with practice. Cerebrovasc Dis. 2005;20 Suppl 2:159-68.

38. Hossmann KA. Experimental models for the investigation of brain ischemia. Cardiovasc Res. 1998 Jul;39(1):106-20.

39. Alonso de Lecinana M, Diez-Tejedor E, Carceller F, Roda JM. Cerebral ischemia: from animal studies to clinical practice. Should the methods be reviewed? Cerebrovasc Dis. 2001;11 Suppl 1:20-30.

40. Hunter AJ, Green AR, Cross AJ. Animal models of acute ischaemic stroke: can they predict clinically successful neuroprotective drugs? Trends Pharmacol Sci. 1995;16(4):123-8.

41. O'Collins VE, Macleod MR, Donnan GA, Horky LL, van der Worp BH, Howells DW. 1,026 experimental treatments in acute stroke. Ann Neurol. 2006 Mar;59(3):467-77.

42. Traystman RJ. Animal models of focal and global cerebral ischemia. Ilar J. 2003;44(2):85-95.

43. DeVries AC, Nelson RJ, Traystman RJ, Hurn PD. Cognitive and behavioral assessment in experimental stroke research: will it prove useful? Neurosci Biobehav Rev. 2001 Jun;25(4):325-42. 
44. Bouet V, Freret T, Toutain J, Divoux D, Boulouard M, Schumann-Bard P. Sensorimotor and cognitive deficits after transient middle cerebral artery occlusion in the mouse. Exp Neurol. 2007 Feb;203(2):55567.

45. Schallert T. Behavioral tests for preclinical intervention assessment. NeuroRx. 2006 Oct;3(4):497-504.

46. Willing $A E$, Jiang L, Nowicki $P$, Poulos $S$, Milliken M, Cahill DW, et al. Effects of middle cerebral artery occlusion on spontaneous activity and cognitive function in rats. Int J Neurosci. 2002 May;112(5):50316.

47. Roof RL, Schielke GP, Ren X, Hall ED. A comparison of long-term functional outcome after 2 middle cerebral artery occlusion models in rats. Stroke. 2001 Nov;32(11):2648-57.

48. Yonemori F, Yamaguchi T, Yamada H, Tamura A. Spatial cognitive performance after chronic focal cerebral ischemia in rats. J Cereb Blood Flow Metab. 1999 May;19(5):483-94.

49. Wahl F, Allix M, Plotkine M, Boulu RG. Neurological and behavioral outcomes of focal cerebral ischemia in rats. Stroke. 1992 Feb;23(2):267-72.

50. Carmichael ST. Rodent models of focal stroke: size, mechanism, and purpose. NeuroRx. 2005 Jul;2(3):396-409. 


\section{Chapter \\ In vivo visualization of vascular leakage in photochemically induced cortical infarction}

Erik I. Hoff Mirjam G.A. oude Egbrink Viviane V.Th. Heijnen Harry W.M. Steinbusch Robert J. van Oostenbrugge Journal of Neuroscience Methods, 2005; 141: 135-41 


\begin{abstract}
The photothrombotic model for stroke was originally described as a focal cortical infarction resulting from occlusive thrombosis. However, subsequent studies have shown evidence for extravasation of water and proteins using ex vivo techniques. The aim of the present study was to determine the role of vascular leakage in the pathophysiology of photochemically induced infarction in vivo. At several time points after infarct induction, analysis of blood flow and vascular leakage was performed using intravital microscopy and fluorescent labelling of blood plasma. In the first hour following infarct induction, massive vascular leakage of the plasma label occurred inside the lesion core that was destined to become infarcted. Flow had stopped completely in this area at 4 hours after illumination. On the day following infarct induction substantial leakage was still present in the penumbral area, defined as the area immediately surrounding the lesion core where reduced flow velocities were observed. Thus, together with the formation of occlusive thrombi, vascular leakage is an important factor in the pathophysiology of photothrombotic stroke.
\end{abstract}




\section{INTRODUCTION}

The use of a photochemical reaction to produce focal cortical infarction in the rat brain was first described in 1985 [1]. Although the use of light-dye reactions to initiate platelet aggregation in the cerebral microcirculation had been described earlier [2], Watson et al. [1] were the first to advocate this photochemical reaction as a means of mimicking cerebrovascular occlusive disease i.e. ischemic stroke. By using transcranial illumination with a filtered light source in combination with intravenous injection of a photosensitive dye, they were able to produce vascular thrombosis. Light microscopy and scanning electron microscopy showed intravascular thrombotic material, red blood cell stasis, and platelet aggregates adhering to luminal surfaces inside pial and parenchymal vessels within superficial layers of the irradiated cortex. Based on these findings, intravascular thrombosis was deemed responsible for the occurrence of ischemia leading to infarction. Subsequent studies, however, investigated the integrity of the blood-brain barrier [3-8], since the aggregation of platelets was found to be caused by endothelial damage [9]. Histological methods were applied to show that protein extravasation was widely distributed within the illuminated zone whereas extravascular increases in water content were restricted to the irreversibly damaged site, suggesting increased permeability through disruption of the blood-brain barrier by the photochemical reaction.

The aim of the present study was to determine the temporospatial distribution of blood-barrier disruption i.e. vascular leakage in the pathophysiology of photochemical cortical infarction in vivo. This was done with the use of intravital microscopy to visualize microcirculatory cortical blood flow and extravasation of a fluorescent blood plasma label.

\section{MATERIALS AND METHODS}

\section{Animals}

All experimental procedures were approved by the local ethical committee for animal experiments of University Maastricht and met government guidelines. Fifteen young adult Lewis rats (LEW/M, Maastricht, the Netherlands) were obtained from the local animal breeding facilities. The animals were housed in standard type III Makrolon cages, 3 rats per cage, on sawdust bedding in an air-conditioned room at approximately $21^{\circ} \mathrm{C}$ with $45-55 \%$ humidity under a 12/12-h light/dark cycle (lights on from 7.00 to $19.00 \mathrm{~h}$ ). They had ad-libitum access to food and water. At the time of ex- 
perimental procedures the rats were 12 weeks of age and weighed between $300-340$ grams (male) or 170-200 grams (female).

\section{Photochemical infarction}

Animals were sedated with halothane, weighed and subsequently intubated for mechanical ventilation using a UNO Micro Ventilator (UNO Roestvaststaal BV, Zevenaar, The Netherlands). Anaesthesia was maintained with $2 \%$ halothane in a mixture of $70 \%$ nitrous oxide and $30 \%$ oxygen (frequency 90 times $/ \mathrm{min}$, volume setting 12 ). Body temperature was monitored and regulated using a rectal probe and waterheated pad. A tail vein cannula was inserted for intravenous injection of the photosensitive agent. The scalp was incised and periosteum removed to expose the skull. A perforated piece of filtration paper was placed over the right parietal bone, centred at $2.7 \mathrm{~mm}$ posterior and $2.7 \mathrm{~mm}$ lateral to Bregma's point, to absorb possible leakage of blood. The light source (diameter $2.4 \mathrm{~mm}$ ) was positioned at right angles to the skull surface in the centre of the perforated filtration paper (Figure 1).

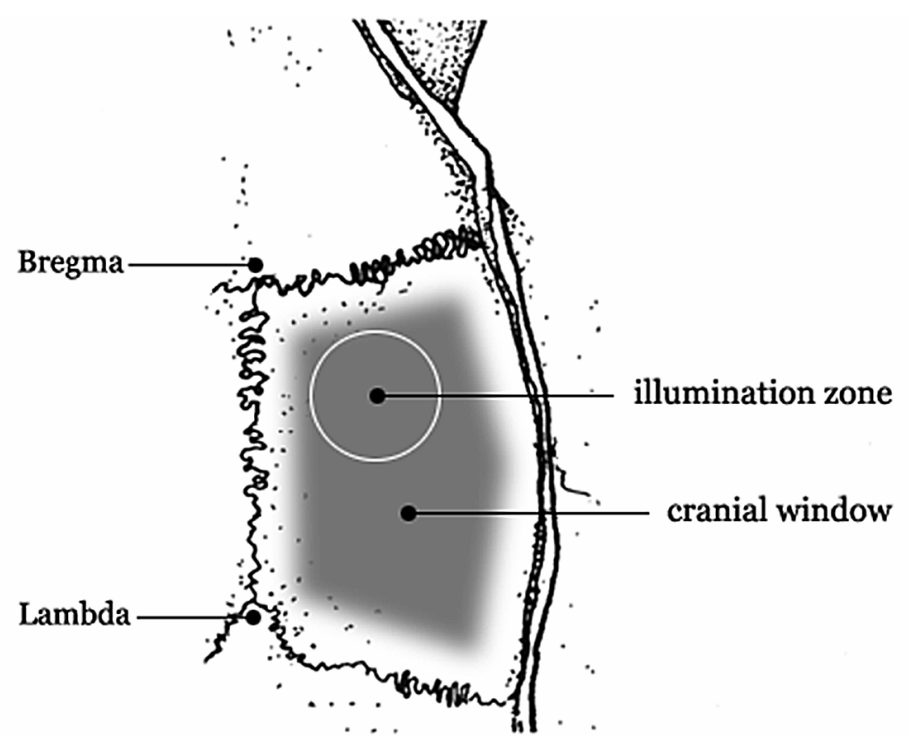

Figure 1. Illustration depicting the right parietal bone of the rat skull. Beam diameter of the light source $(2.4 \mathrm{~mm}$ ) and position of illumination (centred at $2.7 \mathrm{~mm}$ posterior and $2.7 \mathrm{~mm}$ lateral to Bregma) are shown as a white circle; the cranial window is shown in shaded grey. Bregma lies at the intersection of the coronal and sagittal sutures; Lambda lies at the intersection of the occipital and sagittal sutures. 
The irradiating apparatus was custom-made by Instrument Development Engineering \& Evaluation (IDEE, University Maastricht) from a diode-pumped solid-state laser (beam diameter: 0.6mm; wavelength: $532 \mathrm{~nm}$; JDS Uniphase, Germany) with a $4 \mathrm{x}$ optical laser lens beam expander, mounted on an adjustable hydraulic arm. Power output was measured at $12.3 \mathrm{~mW}$, averaging a calculated beam intensity of 300 $\mathrm{mW} / \mathrm{cm} 2$. Each animal was intravenously injected with $20 \mathrm{mg} / \mathrm{kg}$ bodyweight Erythrosin B (Sigma-Aldrich) dissolved in a sterile solution of $0.9 \%$ sodium chloride and subjected to $0.2 \mu \mathrm{m}$ filtration, followed by flushing with $0.9 \%$ sodium chloride to a total volume of $1500 \mu \mathrm{l}$ over a 2-minute period. Then the cover of the light source was removed and the skull was illuminated for 2.5 minutes. Additional cooling of the skull surface to prevent heat-induced cortical damage was deemed unnecessary, given the beam intensity to be below $600 \mathrm{~mW} / \mathrm{cm} 2[1,10]$.

\section{Intravital microscopy}

Intravital microscopy was performed within the first hour after induction of photochemical infarction, 4 hours after induction, or the following day $(n=5$ for each time point). Animals were sedated through an intraperitoneal injection of xylazin (12 $\mathrm{mg} / \mathrm{kg}$, CEVA Santé Animale), and anesthetized with an intraperitoneal bolus injection of ketamin (60 mg/kg, Eurovet Animal Health). Anaesthesia was maintained with ketamin $(20 \mathrm{mg} / \mathrm{kg}$ ), administered subcutaneously every 30 minutes. Animals spontaneously breathed normal air. Throughout these experiments, rectal temperature was monitored and maintained at $36.5-37.5^{\circ}$ Celsius by a feedback regulated heating lamp. A cannula was placed in the right femoral vein for the administration of a fluorescent blood plasma label. The animals that were imaged within 1 hour after infarct induction, received Erythrosin B via the femoral vein cannula instead of a lateral tail vein cannula. The head of each rat was fixed in a stereotaxic frame (David Kopf Instruments). Using a dentist drill (EM 2900 A; Berger Technik), craniotomy of the right parietal bone was performed 1-2 $\mathrm{mm}$ inside coronal, sagittal, and lambdoid sutures and attachment of temporal muscle, leaving the dura mater intact (Figure 1). Additionally, in several animals the left parietal bone was also removed for control measurements in normal vasculature.

Microscopic observations of the brain microvessels were performed with an intravital microscope (all components: Leitz, Wetzlar, Germany). A Ploemopak 2.2 incident illuminator (tube factor 1.25x), a mercury arc (100 W), and either a 6.5 objective (numeric aperture (n.a.) 0.18 ) or an LL25 objective (n.a. 0.35) were used for bright-field microscopy. Application of a POL cube (polarizer, 50\% mirror, and crossed analyzer) minimized direct light reflections from the brain surface. Fluores- 
cence microscopy was performed using a SW 25 objective (n.a. 0.6). Images were projected onto a CCD camera, or in case of fluorescence microscopy onto an intensified CCD camera (C2400-8; Hamamatsu Photonics, Hamamatsu City, Japan), and recorded on videotape or DVD. While keeping the dura mater moist with a sterile saline solution, the cranial window was first scanned back-and-forth from anterior to posterior using the $6.3 x$ objective to obtain a general overview and, subsequently, at $25 x$ magnification for a more detailed visualization. In areas where blood flow was present, arterioles or venules were identified according to the direction of flow. That is, convergence of flow is only seen in venules, whereas divergence of flow indicates arterioles. Subsequently, blood vessels were selected for fluorescent imaging of blood plasma. To this purpose, FITC-labelled dextrans (10-70 kDa) were administered $(0.5 \mathrm{ml}$ of $5 \mathrm{mg} / \mathrm{ml}$ saline, i.v.) that were visualized using filter set 12 (excitation BP 450-490 nm; dichroic mirror RKP 510; barrier LP515). After injection of the fluorescent plasma label, video or DVD images of one or two vessels per animal were recorded per time point; total recording time was 20 minutes.

\section{Image analysis}

Off-line, flow patterns in arterioles and venules in the cranial window were assessed at $25 x$ magnification. Per optical field $(310$ by $230 \mu \mathrm{m})$ the flow pattern of all vessels was scored semi-quantitatively as either "fast flow" (+) if no individual red blood cells could be discriminated in the blood stream, "slow flow" (=) when individual, moving red blood cells were observed, or "no flow" (-). If different flow patterns occurred in the same optical field, flow in small vessels $(<30 \mu \mathrm{m})$ was noted first and separated from flow in larger vessels by a forward slash. At each border of the cranial window at least two optical fields were discarded from analysis, because of artefacts caused by the experimental procedure (e.g. drilling). Exemplary results are shown in Figure 2. Because of inter-individual differences in size of the cranial window, no group averaging was performed on the flow pattern data. However, based on these flow patterns, data on vascular leakage could afterwards be interpreted for every single animal as being recorded in normal areas (fast flow areas), peri-infarct or penumbral areas (slow flow), or infarcted areas (no flow). 


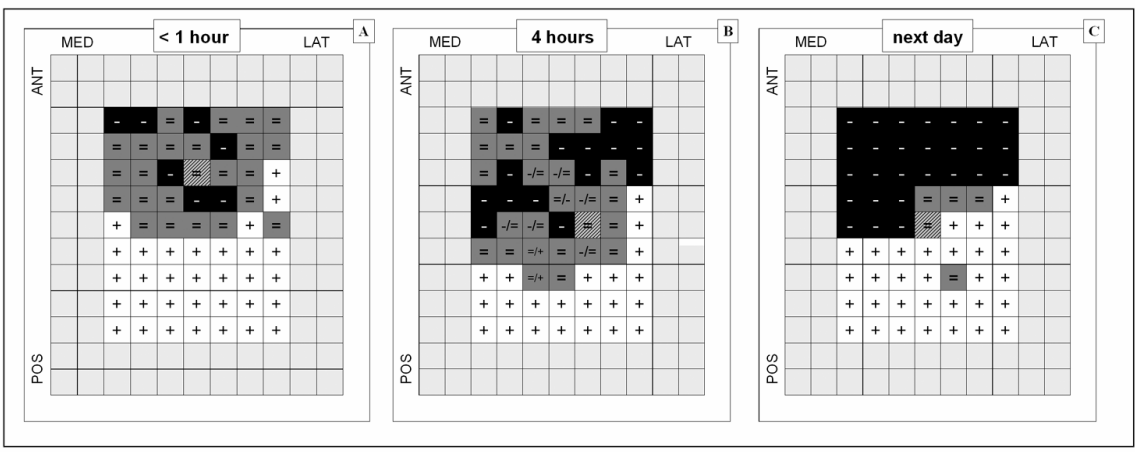

Figure 2. Quantification of flow patterns inside the cranial window. Per optical field, the flow pattern of the vessels was scored semi-quantitatively as either "fast flow" (+), "slow flow" (=) or "no flow" (-). The optical fields showing a stripe pattern indicate the areas in which quantification of vascular leakage was performed. For every time point observed, one exemplary result is given: within 1 hour after infarct induction (A), after 4 hours (B), and the next day (C). At earlier time points penumbral areas were usually larger in size, and frequently, various flow patterns per optical field were observed, especially after 4 hours. On the day following illumination, a clear distinction was visible between infarcted and non-infarcted areas. Abbreviations: ANT = anterior, POS = posterior, MED = medial, LAT = lateral.

Digitized video images or DVD images were imported in Adobe Photoshop to enable quantification of fluorescent plasma label leakage from brain microvessels. The use of Adobe Photoshop for quantitative image analysis of cerebral microcirculation was adapted from [11]. Three identical circular areas were drawn that covered the complete intravascular space (one circle) and its immediate surroundings (two circles on opposite sides of vessel). The diameter of each circle equalled vessel diameter. Special care was taken with the placement of the circles in order to minimize possible interference from neighbouring vessels. The average of mean grey values in the extravascular circles was divided by the mean of the grey values inside the intravascular circle, resulting in ratios of fluorescence levels outside and inside the blood vessel. Such ratios were determined in every animal over the 20-minute time frame after FITC-dextran administration, and thus, represent the level of plasma label leakage from the vessels. As mentioned before, one or two vessels (generally one arteriole and a venule) were recorded per animal per time point, and twice as much when control measurements were performed on normal vasculature. 


\section{Data analysis}

For every post-lesion time point, the fluorescence ratios were averaged per vessel type. Parts of the analyses were performed independently by two authors (EH and MOE). The inter-rater variability of these data was good (kappa 0.8). All data were compared in a repeated measures analysis (SPSS, version 11). Within-subject factor was the time after FITC-dextran injection (Time - 5 levels) and between-subjects factor was vessel type per time point. In order to specify the time point at which arterioles and venules were leaky, pair wise comparisons were made with the estimated marginal means of the corresponding control vessel type using a Sidak adjustment for multiple comparisons $(p=0.05)$.

\section{Histology}

With intravital microscopy through a cranial window, one can only observe the microcirculation at the cortical surface. In addition, pial microvessels are observed that may have different properties in terms of permeability compared to cerebral microvessels [12]. To be able to extend our findings to deeper cortical vessels and blood-brain barrier integrity, we intravenously injected a $2 \%$ Evans Blue dye solution (Sigma-Aldrich) at the end of intravital microscopy [13]. The animal was sacrificed 30 minutes later. For macroscopic analysis of vascular leakage, brains were snapfrozen, cryostated and contingent presence of the blue dye verified.

\section{RESULTS}

Flow pattern

In the animals where craniotomy was performed on the side contralateral to illumination, normal vascular architecture with fast flow patterns was observed. The posterior part of the cranial window that had not been illuminated also showed normal vascular architecture with fast flow patterns, independently of the time period following induction of photochemical infarction. Figure 2 illustrates that within the first hour after infarct induction, slow flow as well as no flow was observed in vessels inside the illuminated zone, together with the occasional passage of thrombotic-like material. After 4 hours, vessels with slow flow or no flow were observed as well, but the area with no flow-vessels increased. Strikingly, disturbances in flow were observed more often in smaller vessels (diameter $<30 \mu \mathrm{m}$ ) than in larger ones (diameter $>30 \mu \mathrm{m}$ ). On the day following illumination, flow had stopped in all vessels within the illumi- 
nated zone. Therefore, this zone is referred to as the lesion core. In the immediate surroundings of this lesion core, slow flow was observed after 4 hours. On the following day this area had reduced to only a few optical fields. We refer to this slow flow area, between lesion core and normal flow area, as the penumbra. Within the first hour after induction, we were not yet able to identify this region.

\section{Vascular leakage}

Initial intravital microscopy experiments were aimed at defining the optimal size of FITC-labeled dextrans to be used for quantitative analysis of vascular leakage. With injection of $10 \mathrm{kDa}$ dextrans an almost immediate and massive increase in perivascular fluorescence was seen inside the irradiated zone. After injection of $70 \mathrm{kDa}$ dextrans, fluorescence in this area barely increased. For this reason, all further experiments were conducted using $40 \mathrm{kDa}$ FITC-labeled dextrans.

Analysis of images obtained from venules and arterioles of the hemisphere contralateral to the illumination site showed no increase in fluorescence ratio (Figure 3A), which means that normal non-infarcted cerebral microvessels are impermeable to 40 kDa dextrans. During the 20-minute imaging sessions, even a slight decrease was found, indicating natural clearance of the plasma label from the blood circulation. Data obtained from arterioles and venules inside the illuminated area are presented for every post-lesion time point (Figures 3B-C). Since blood flow inside the lesion core was only detectable immediately after infarct induction, no data could be obtained from this area at later time points. Within the first hour of illumination, the ratio of extravascular over intravascular fluorescence of both arterioles and venules inside the lesion core increased from control values to $0.8-1.0$ over the 20-minute recording period. These data indicate that inside the lesion core both arterioles and venules become leaky within the first hour after photochemical induction of cortical infarction. As stated before, fluorescent imaging of vessels inside the lesion core was not possible after 4 hours and on the day following induction of infarction, because of a lack of blood flow. At these time points a normal and regular distribution of the plasma label was seen both in arterioles and venules of the penumbra. At 4 hours a relative increase in extravascular fluorescence was observed in arterioles as well as venules. At the end of the 20-minute recording time, the ratios were found to be similar to those found in the lesion core within the first hour (0.75-1.0). On the day following infarct induction, arterioles and venules again showed a similar increase in fluorescence ratios after administration of the plasma label (to 0.90 and 0.68 , respectively). These data suggest that vessels are leaky in the penumbra as well. On the other hand, no signs of thrombosis were observed. 

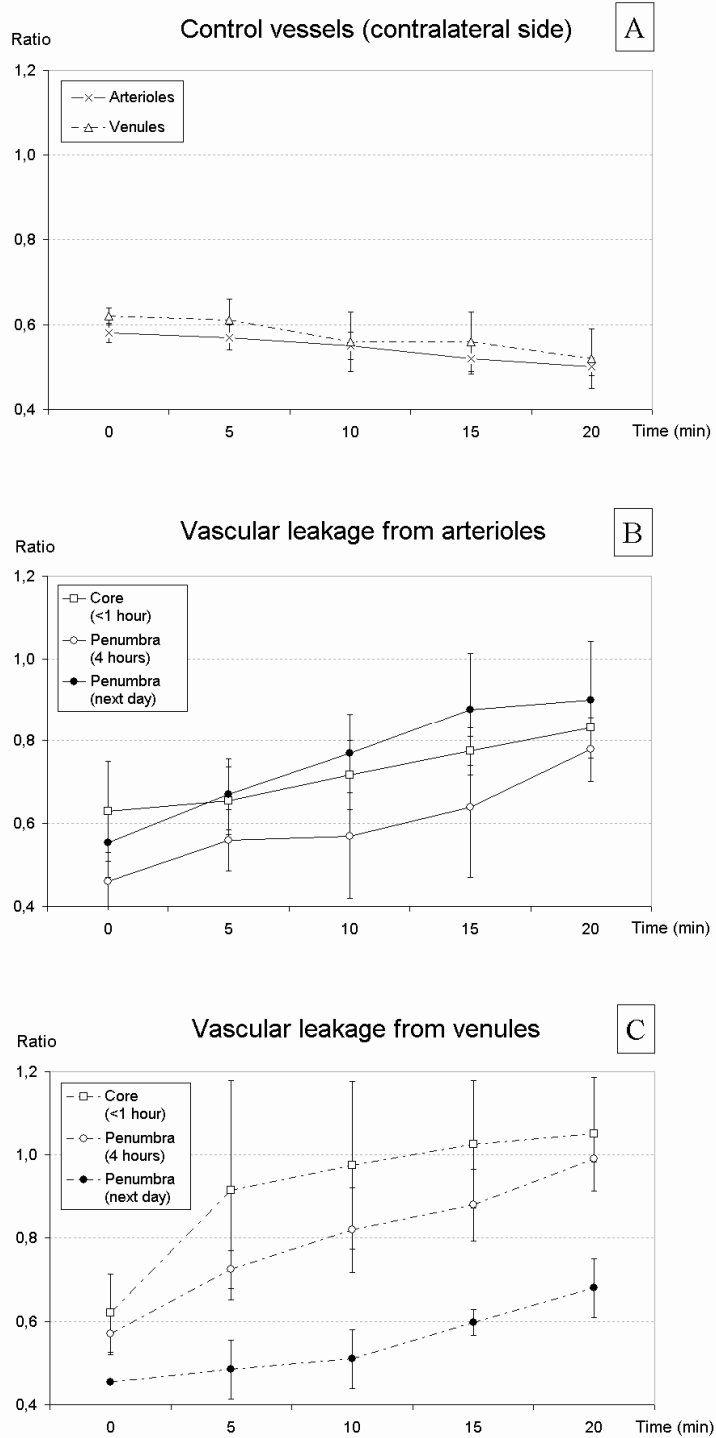

Figure 3. Ratios of extravascular over endovascular fluorescence in time after injection of FITC-labeled dextran $(40 \mathrm{kDa})$. Based on flow pattern and location of the optical field inside the cranial window, fluorescence ratios in every single rat could retrospectively be assigned to either lesion core or penumbral areas. Individual measurements were averaged per vessel type for every time point after infarct induction. Measurements from control vessels were obtained from a cranial window on the contralateral side of illumination $(A)$ and show a slight decrease in fluorescence ratio, suggestive of natural clearance from the blood stream. By contrast, an increase in this ratio is seen in arterioles and venules at every post-lesion time point (B and $C)$. 
Statistical analysis confirmed the observation of vascular leakage. Repeatedmeasures analysis showed a significant effect of time after FITC-injection $(F(4)=133.24, p<0.001)$. Pairwise comparison between arterioles demonstrated that leakage was still present after one day of infarct induction $(p<0.05)$, whereas the same comparison for venules revealed significance at 4 hours $(p<0.02)$ and a trend at 1 hour $(p=0.10)$. No differences were found between leakage ratios at baseline.

Vascular leakage was also confirmed macroscopically after intravenous injection of the dye Evans Blue. In undamaged brain areas like cerebellum and contralesional hemisphere no dye leakage was found. At all time points after infarct induction, however, Evans Blue was observed to have extravasated at the lesion site on the cortical surface, and was often found to be dispersed a few millimetres over the ipsilesional hemisphere. This is in accordance with the intravitally obtained data. In deeper cortical layers, Evans Blue was present throughout the extension of the lesion within 1 hour of infarct induction, and in its immediate surroundings (the penumbra) up until one day after infarct induction. The lesion core did not show leakage of Evans Blue from 4 hours onwards, which is in accordance with the absence of flow in vessels within this area.

\section{Discussion}

The present study investigated vascular permeability of the cortical microcirculation after photochemically induced cortical infarction in vivo. This was done using intravital microscopy and fluorescent labelling of blood plasma, which enables real-time visualization and quantification of changes in flow and permeability in different vessel types. As a result, the temporospatial distribution of blood-barrier disruption could be determined. We have found extensive vascular leakage of the $40 \mathrm{kDa}$ plasma label from superficial, cortical arterioles and venules within the illuminated area and its penumbra up to one day following induction of infarction. In agreement with findings by Belayev et al. [13], the extravasation of Evans Blue within the entire ischemic area shows that these results are applicable to deeper cortical microcirculation as well and reflect disruption of the blood-brain barrier.

In previous studies using histological methods, it has been evidently shown that blood-brain barrier disruption is a fundamental mechanism in the development of photochemically induced cortical infarction. Dietrich et al [3] demonstrated that a complete cortical lesion could be obtained in the absence of thrombosis by cooling the brain by $3^{\circ} \mathrm{C}$. Furthermore, Haseldonckx et al. [7] described that minimizing the photochemical challenge to endothelial membranes modifies the photothrombotic stroke model into one that models the ischemic penumbra. Using a lower concentra- 
tion of the photosensitizer and a shorter duration of illumination at lower output, they showed limited congestion of microvessels by erythrocytes and extensive blood-brain barrier leakage of ultrasmall gold particles within minutes to 1 hour after illumination. Thereafter, progressive intravascular congestion occurred, which was attributed to extravasation and consequent formation of perivascular oedema. These findings were compared to those seen when photochemical induction of infarction was performed at higher concentration of photosensitizer with longer illumination at higher output. Almost immediate congestion of cortical microvessels was found, whereas leakage of gold particles was mainly situated at the margins of the thrombotic core.

Although it seems that these findings contradict to the role of vascular occlusion by platelets in the original description of the model, we recognize both 'thrombosis' and 'leakage' as well-accepted events in the pathophysiology of the photochemical model. These events largely depend on the concentration of the photosensitive agent, irradiation parameters and physiological circumstances at the time of the photochemical procedure. Thus, it might well be that the sequence of events at higher photochemical challenge takes place in a shorter time frame. An alternative explanation, as derived from our findings, is that aggregation of platelets may largely account for vascular occlusion in small vessels $(<30 \mu \mathrm{m})$ whereas vascular leakage mainly contributes to the stasis of blood flow in larger ones. In accordance, we have found that flow decreased or stopped first in small vessels 4 hours after infarct induction. And although we did observe the occasional passage of thrombotic-like material in larger vessels, especially inside the lesion core, flow in these vessels was still preserved for at least 4 hours after infarct induction. However, since we were unable to monitor brain temperature, cooling of the brain surface may have occurred. This could have resulted in a decreased aggregation of platelets [3], which in turn, may have led to a relative overemphasis of the vascular leakage component in this study. It has been stated that, in this animal model, the formation of occlusive thrombi occurs before disruption of the blood-brain barrier [14], suggesting that these thrombi are the cause of ischemia ultimately leading to infarction. Whereas we did observe vascular irregularities and possible thrombus formation on the endothelial wall in the illuminated area within the first hour after infarct induction, microcirculatory blood flow was still present together with a massive leakage of the $40 \mathrm{kDa}$ plasma label, indicative of early damage of the blood-brain barrier. Therefore, the lack of blood flow in the illuminated zone after 4 hours may not be caused by the formation of occlusive thrombi alone, but also by massive leakage of proteins and fluids across a damaged blood-brain barrier. Together, this will lead to a reduction in blood flow and eventually to stasis of red blood cells in pial and parenchymal cortical vessels, as was originally described by Watson et al. [1]. 
Secondary ischemia due to compression of microvessels by cerebral oedema is used as an explanation of gradually expanding infarction outside the original area of illumination $[7,10]$. The relationship between cerebrovascular permeability and cerebral oedema was described by Laursen et al. [5], who showed increased permeability of the blood-brain barrier to serum proteins from two hours onwards after photochemical induction of cortical infarction. Although our results demonstrated that blood vessels in the immediate surroundings of the lesion core are still leaky one day after photochemical challenge, we could not confirm a substantial expansion of infarction. Based on our findings, this is due to (a) the preservation of blood flow outside the illuminated area, and (b) the extravasation of Evans Blue, which appeared to be more widespread at 4 hours than one day after infarct induction. After photochemical challenge, however, blood-borne factors are known to be released and are proposed to increase vascular permeability [15]. This might explain why higher concentrations of photosensitive dye and longer illumination duration produce larger infarction, and conversely, the lack of expansion found in our application of the model.

In conclusion, we have found extensive leakage of a large protein from arterioles and venules in the superficial cortical microcirculation after photochemically induced infarction. In addition, we have shown that these results are applicable to deeper cortical microcirculation. Thus, apart from occlusive thrombi, the pathophysiology of photothrombotic stroke also includes substantial disruption of the blood-brain barrier. Moreover, differential effects may exist with respect to vessel diameter. Small vessels may be more prone to early intravascular occlusion through thrombi formation, whereas in larger vessels vascular leakage continues for longer periods of time. 


\section{REFERENCES}

1. Watson BD, Dietrich WD, Busto R, Wachtel MS, Ginsberg MD. Induction of reproducible brain infarction by photochemically initiated thrombosis. Ann Neurol. 1985;17(5):497-504.

2. Rosenblum WI, El-Sabban F. Platelet aggregation in the cerebral microcirculation: effect of aspirin and other agents. Circ Res. 1977 Mar;40(3):320-8.

3. Dietrich WD, Busto R, Watson BD, Scheinberg P, Ginsberg MD. Photochemically induced cerebral infarction. II. Edema and blood-brain barrier disruption. Acta Neuropathol (Berl). 1987;72(4):326-34.

4. Grome JJ, Gojowczyk G, Hofmann W, Graham DI. Quantitation of photochemically induced focal cerebral ischemia in the rat. J Cereb Blood Flow Metab. 1988;8(1):89-95.

5. Laursen H, Hansen AJ, Sheardown M. Cerebrovascular permeability and brain edema after cortical photochemical infarcts in the rat. Acta Neuropathol. 1993;86(4):378-85.

6. Van Reempts $\mathrm{J}$, Borgers M. Histopathological characterization of photochemical damage in nervous tissue. Histol Histopathol. 1994 Jan;9(1):185-95.

7. Haseldonckx M, van Bedaf D, van de Ven M, van Reempts J, Borgers M. Vasogenic oedema and brain infarction in an experimental penumbra model. Acta Neurochir Suppl. 2000;76:105-9.

8. Gadamski R, Barskow IW, Szumanska G, Wojda R. Blood-brain barrier disturbances and morphological changes in rat brain after photochemically induced focal ischaemia. Folia Neuropathol. 2001;39(3):15561.

9. Dietrich WD, Watson BD, Busto R, Ginsberg MD, Bethea JR. Photochemically induced cerebral infarction. I. Early microvascular alterations. Acta Neuropathol. 1987;72(4):315-25.

10. Watson BD. Animal models of photochemically induced brain ischemia and stroke. In: Ginsberg MD, Bogousslavsky J, editors. Cerebrovascular Disease - Pathophysiology, Diagnosis and Treatment. Cambridge, MA: Blackwell Science; 1998. p. 52-73.

11. Brunner J, Krummenauer F, Lehr HA. Quantification of video-taped images in microcirculation research using inexpensive imaging software (Adobe Photoshop). Microcirculation. 2000 Apr;7(2):103-7.

12. Allt G, Lawrenson JG. Is the pial microvessel a good model for blood-brain barrier studies? Brain Res Brain Res Rev. 1997 Jun;24(1):67-76.

13. Belayev L, Busto R, Watson BD, Ginsberg MD. Post-ischemic administration of HU-211, a novel noncompetitive NMDA antagonist, protects against blood-brain barrier disruption in photochemical cortical infarction in rats: a quantitative study. Brain Res. 1995;702(1-2):266-70.

14. Gajkowska B, Frontczak-Baniewicz M, Gadamski R, Barskov I. Photochemically-induced vascular damage in brain cortex. Transmission and scanning electron microscopy study. Acta Neurobiol Exp. 1997;57(3):203-8.

15. Dietrich WD, Prado R, Watson BD. Photochemically stimulated blood-borne factors induce blood-brain barrier alterations in rats. Stroke. 1988;19(7):857-62. 


\section{Dissociable effects in reaction time}

performance after unilateral cerebral infarction: a comparison between the left and right frontal cortices in rats

Erik I. Hoff Arjan Blokland

Kris Rutten

Harry W.M. Steinbusch Robert J. van Oostenbrugge 


\begin{abstract}
Reaction time performance reflects the speed of information processing, both in humans and lower vertebrates like the rat. The present study compared reaction time performance in rats following unilateral infarction to the frontal cortex. The objective was to model cognitive impairment as it is seen in humans after stroke. Rats were trained in a reaction time paradigm, after which unilateral cortical infarction was induced photochemically. Reaction time performance was differentially affected after unilateral infarction to either the left or right frontal cortex, whereas sham-operation did not result in a significant alteration in reactivity. An overall increase in reaction time of about $10 \%$ was present at four weeks after frontal infarction. In addition, a lateralized reaction time deficit occurred very early after right frontal infarction as an increase of $10-15 \%$ in trials directed towards the contralesional side. Additional analyses showed that these reaction time deficits can be explained differently: the former as a gradual and general decrease in the speed of information processing, whereas the latter shows specific impairment to initiate a contralateral motor response. The former matches well with the mental slowing observed in stroke patients, whereas the latter resembles a neglect phenomenon. We conclude that measuring reaction time performance after frontal cortical infarction in rats could offer a useful tool to model particular human cognitive impairments following cerebral infarction.
\end{abstract}




\section{INTRODUCTION}

Cerebrovascular disease is the second most common cause of acquired cognitive impairment and dementia [3]. A general slowing in the speed of information processing is frequently encountered, which is most prominent when time pressure is part of a task performance [4-8]. Both in humans and in lower vertebrates like the rat, the latency to respond (reaction time) is a direct measure of information processing speed [9]. In clinical and experimental neuropsychology, therefore, reaction time is an accepted measure in the assessment of cognitive function. The use of reaction timebased parameters has contributed greatly to the present knowledge of information processing in humans. In animal research, reaction time testing has mostly been limited to studies on mental slowing in aging and to the role of the striatum in the facilitation of movements (Parkinson's disease). However, it could offer the possibility to study basic mechanisms behind disturbances of higher cortical functioning after cerebral infarction. Thus far, reaction time experiments into information processing after cortical damage have mostly studied bilateral cortical lesioning [10-18] or unilateral cortical lesioning of a single hemisphere [19-22]. To our knowledge, no studies have been published in which a direct comparison is made between reaction time performance after unilateral lesions to the left or the right frontal cortex.

The objective of the present study was to investigate whether the general slowing of cortical information processing speed in stroke patients can be modelled. To this end, we investigated the effects of unilateral cortical infarction on reaction time performance in rats. We focussed on the frontal cortex, as it is both frequently involved in cerebrovascular disease and in the temporal organization of behaviour [23, 24]. Since lateralization of brain functions is known to occur within the rodent brain [25-30], a direct comparison was made between reaction time performance after unilateral lesions to the left versus the right frontal cortex.

\section{MATERIALS AND Methods}

\section{Animals}

All animal surgical and maintenance procedures were approved of by the local ethical committee of Maastricht University and are in agreement with governmental guidelines. A total of thirty-two male Lewis rats were used, which at the time of the surgical procedure were 12 weeks of age and weighed 250-300 g. The rats were housed individually in standard type III Makrolon cages, on sawdust bedding in an airconditioned room of about $21^{\circ} \mathrm{C}$ with $45-55 \%$ humidity under a reversed light/dark 
cycle (lights on from 19.00 to $07.00 \mathrm{~h}$ ) and had ad libitum access to water. To prevent a motivational bias during behavioural testing, the rats were food-restricted and given 12-14 g laboratory chow per day. In the weekends, the rats had ad libitum access to food up until 12 hours prior to testing. In doing so, the weight of the animals was kept at $90 \%$ of a freely-fed reference group. We compared unilateral infarction to the left frontal cortex and the right frontal cortex ( $n=10$ in each group). A group of shamoperated animals served as controls $(n=12)$.

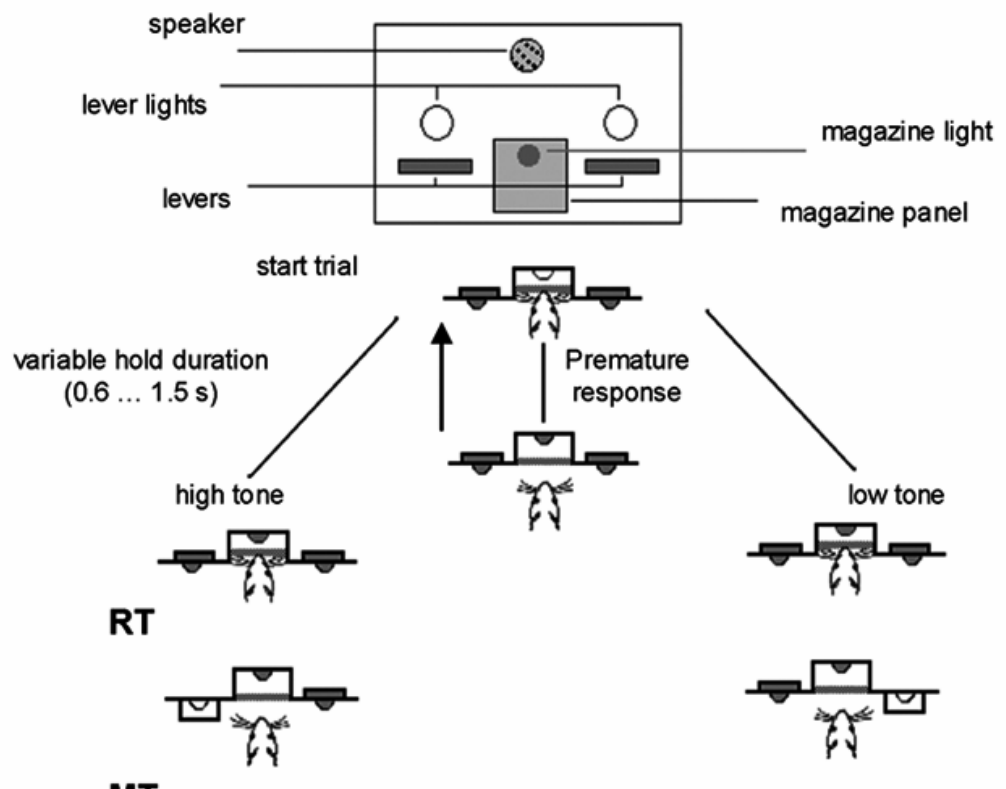

MT

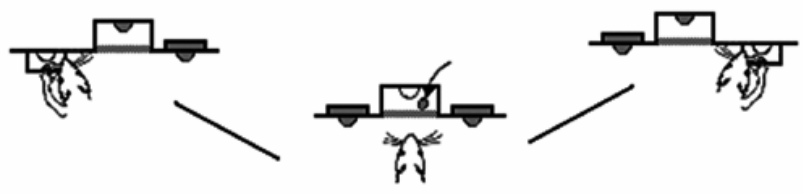

Figure 1. Schematic representation of the behavioural paradigm, showing the operating wall of the Skinner box (top) and the series of stages in the reaction time task. Abbreviations: RT (reaction time) $=$ the difference in time elapsed between auditory stimulus and panel release. MT (motor time) $=$ the difference in time elapsed between panel release and level press. PR (premature responses) $=$ panel release before the end of the hold duration. 


\section{Behavioural training}

The rats were trained to perform a reaction time task [31], of which Figure 1 shows a schematic representation. First, the rats learned to push back a hinged panel to retrieve a food reward (45 $\mathrm{mg}$ food pellets, Bioserve Inc.). The next stage of training consisted of continuous reinforcement training, in which either the left or the right lever was inserted into the conditioning chamber after the rat had pressed the hinged panel of the food tray. Then, the rats had to push the hinged panel for a longer duration until one of the levers was inserted into the chamber.

A randomly chosen time period termed 'hold duration' was used (600-1500 ms, increment $100 \mathrm{~ms}$ ). When the hold duration had elapsed an auditory stimulus was presented. A high tone $(10 \mathrm{kHz}, 80 \mathrm{~dB})$ required pressing the left lever whereas a low tone $(2.5 \mathrm{kHz}, 80 \mathrm{~dB})$ required pressing the right lever. At the final stage of training, a $50 \%$ partial reinforcement schedule was applied. A single session lasted until 60 trials had been performed within a maximum of 30 minutes. Baseline measurements were calculated after the rats showed stable performance, that is, no change in reaction time performance on five successive training days. This was done by averaging the last six sessions of the alternate three weekdays prior to surgery to average out a possible bias in testing (time of the week).

The main behavioural outcome parameters used for the statistical analyses were: (1) Reaction Time (RT), the latency between tone onset and panel release, is determined by a simple movement (retraction of the head) and dependent on attentional function, sensory processing, response selection and motor initiation; (2) Motor Time (MT), being the latency between panel release and lever press, involves a skilled bodily movement reflecting motor execution; and (3) Premature Responses (PR) is a measure of response inhibition. PR were scored when the rat released the panel before the end of the hold duration. A ratio was calculated by dividing the number of premature responses by the total number of trials.

\section{Surgery}

Within each experiment, body weight and reaction time were matched in both lesion groups prior to surgery. Cortical infarction was induced by photochemically initiated thrombosis [32, 33]. This method was chosen because it produces highly reproducible, circumscript damage of cortical structures. Animals were anaesthetized with $2 \%$ halothane in a mixture of $70 \%$ nitrous oxide and $30 \%$ oxygen, and allowed to breathe spontaneously. Body temperature was feedback-controlled between $36.5-37.5^{\circ} \mathrm{C}$ by a rectal probe and water-heated pad. In order to expose the skull, the scalp was 
incised and the periost removed. The light source, with a diameter of $2.4 \mathrm{~mm}$, was positioned perpendicular to the skull surface within the centre of a perforated filtration paper that absorbed any leakage of blood. The laser beam was positioned at $2.7 \mathrm{~mm}$ anterior and $2.7 \mathrm{~mm}$ lateral to Bregma's point for infarction of the frontal cortex. Through a cannula inside a lateral tail vein, each animal was intravenously administered Erythrosin B $(20 \mathrm{mg} / \mathrm{kg})$ dissolved in a sterile solution of $0.9 \%$ sodium chloride and subjected to $0.2 \mu \mathrm{m}$ filtration, followed by flushing with $0.9 \%$ sodium chloride to a total volume of $1500 \mu \mathrm{l}$ over a 2-minute period. Then the cover of the light source was removed and the skull illuminated for 2.5 minutes $(300 \mathrm{~mW} / \mathrm{cm} 2)$. In shamoperated animals, the order of dye injection and illumination was reversed.

\section{Behavioural testing after surgery}

We found the animals to behave normally already a few hours after lesioning: grooming, gross motor function, eating and drinking. From the first day after surgery, the rats were able to complete the reaction time paradigm. Thus, no recovery period was needed after surgery and we decided to train and test continuously. The rats were tested twice daily on three alternate weekdays for four weeks. Every day, the mean was taken from the two sessions to average out a possible bias in testing (time of day). Every post-lesion time-point was construed from the data of two separate days. To assess a general effect of the surgical procedure, in particular the influence of anaesthesia or the development of oedema on reaction time performance, the data were averaged from the first and third days after surgery or, if a weekend had passed, the first and fourth days after surgery $(D<4)$. In the weeks following surgery, the data of four sessions collected on two days of testing were averaged every week (W1, W2, W3 and W4).

\section{Histology}

At the end of behavioural testing histology was performed on all rat brains in order to verify lesion volume and location. Around the lesion site, the brain was serially cut into $30 \mu \mathrm{m}$ coronal sections and mounted onto slides. For the quantification of infarct volumes every fifth section was stained using a May-Grünwald-Giemsa protocol. The area and depth of the lesion were delineated in every section on a stereological microscopy set-up. Lesion volume (in $\mathrm{mm} 3$ ) was calculated by multiplying area by intersection thickness. 


\section{Statistical analyses}

All statistical analyses were done using SPSS software (version 12). Post-lesion performance in reaction time (RT), motor time (MT) and premature responses (PR) was compared to baseline performance in a between-group analysis using pairedsamples t-testing (statistical significance at $\mathrm{p}<0.05$ ). In addition, the difference between contralesional and ipsilesional response times was deducted from their respective baselines and subjected to one-sample t-testing. For certain post-lesion time points, analyses were performed on RT versus hold duration. This tests the effect of the lesion on the preparation time needed to initiate a response, also known as expectancy or anticipation. To this end, RTs were subdivided into five classes of hold durations, from shortest to longest hold duration: class $1=600 / 700 \mathrm{~ms}$, class $2=$ $800 / 900 \mathrm{~ms}$, class $3=1000 / 1100 \mathrm{~ms}$, class $4=1200 / 1300 \mathrm{~ms}$, and class $5=$ $1400 / 1500 \mathrm{~ms}$. In addition, the total range of RTs was sorted from fastest to slowest reaction times and subsequently subdivided into six classes. Such an RT distribution analysis can be used to evaluate the role of underlying processes involved in reaction time responding $[34,35]$. Lesion volume was compared between groups using an independent-samples t-test.

\section{RESULTS}

Figure 2 shows histological sections of photochemically initiated infarction to the frontal cortex as well as the location and volume distribution of the lesions. Mean lesion volume did not differ significantly between groups: left frontal $1.43 \mathrm{~mm} 3$ (SEM $\pm 0.15)$, and right frontal $1.29 \mathrm{~mm} 3(\mathrm{SEM} \pm 0.27$ ); sham-operated animals were without lesions. Frontal lesions were located in the rostral part of the cortical regions Fr1 and Fr2. Most lesions were transcortical, with the corpus callosum being involved in one animal with a right frontal lesion. This animal was removed from statistical analysis, together with two other animals that did not recover from surgery. The final number of animals per lesion group was: left frontal cortex $(n=9)$, right frontal cortex $(n=9)$, and sham-operated $(n=11)$. No differences in body weight or task completion occurred during the four weeks of behavioural testing after infarction. 

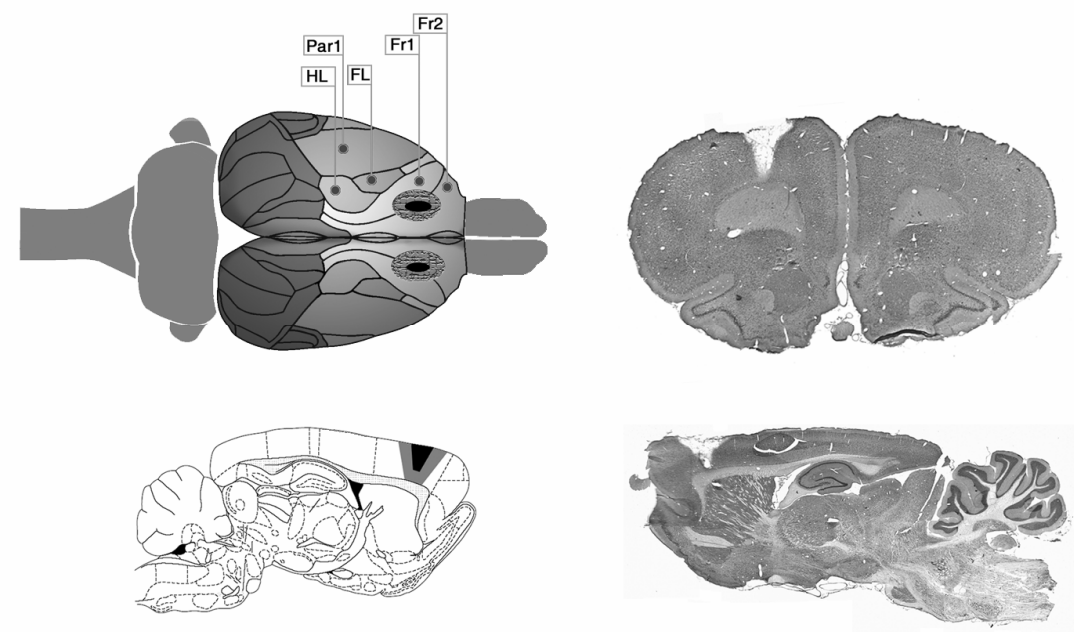

Figure 2. On left: dorsal and sagittal view of the rat brain showing the variation in lesion extent (grey circle) and the greatest common divisor of all lesions in a group (black circle). On right: histological sections with May-Grünwald-Giemsa staining showing a coronal and sagittal view of photochemically infarction to the frontal cortex. Frontal region: Fr $1=$ frontal area 1 (or primary motor cortex), Fr2 = frontal area 2 (also known as medial agranular cortex). Parietal region: $\mathrm{FL}=$ sensorimotor area of forelimb, $\mathrm{HL}=$ sensorimotor area of hindlimb, Par1 = parietal area 1. The dorsal view was adopted from Zilles and Wree [1], the sagittal view from Paxinos and Watson [2].

\section{Motor times and premature responses}

Figure 3 shows the results of behavioural testing after surgery. Unilateral infarction to the right frontal cortex significantly decreased overall motor time at W3 and W4, and showed a trend towards a decrease after infarction of the left frontal cortex at W4 $(p=0.06)$. Motor time performance did not change following sham-operation. No differences were observed between ipsilesional and contralesional response times in the frontal lesion groups.

The proportion of premature responses decreased primarily after sham operation ( $p<0.01$ at all post-surgery time-points). Unilateral infarction to the right frontal decreased premature responses only at W3 and W4 $(p<0.05)$, and showed trends toward a decrease after infarction to the left frontal cortex at D7 $(p=0.05)$ and W4 $(p=0.06)$. 

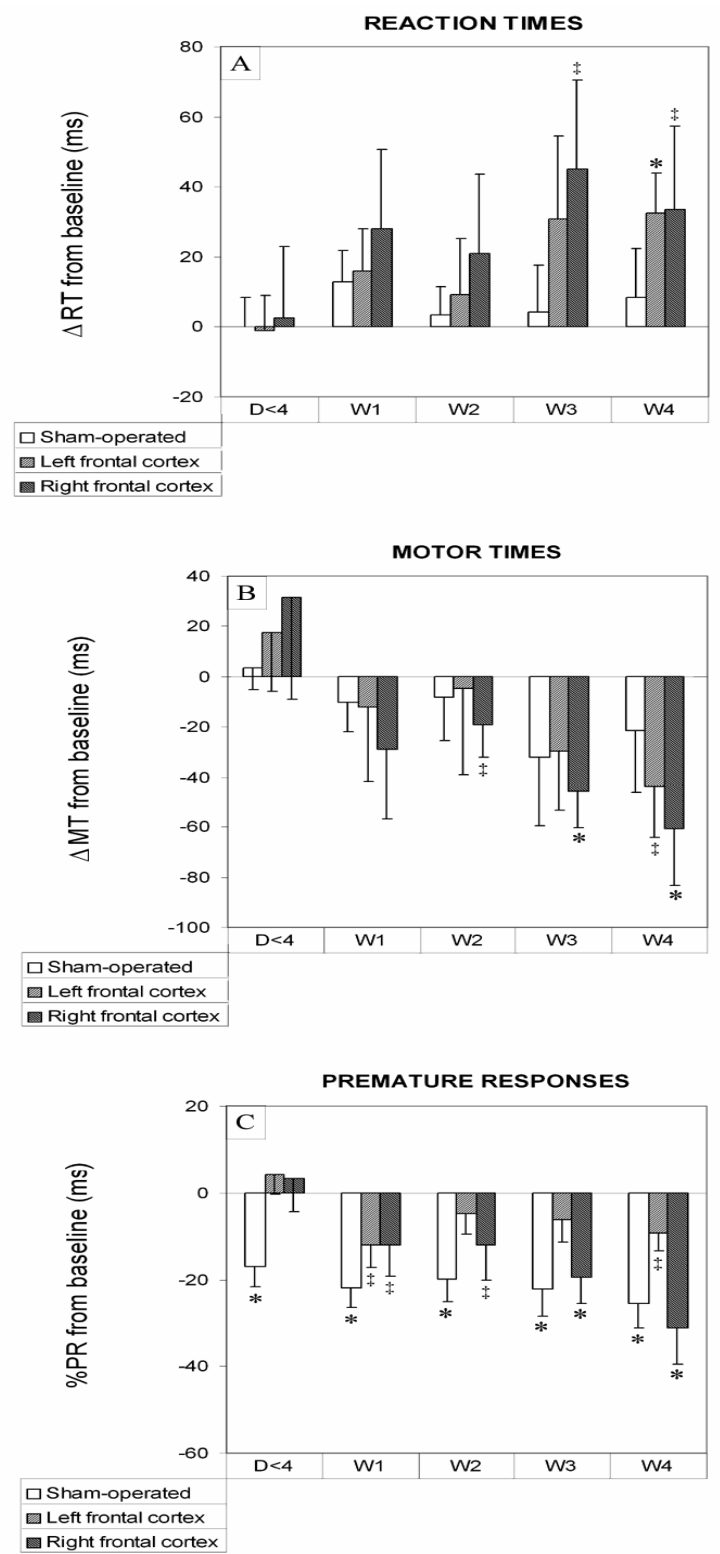

Figure 3. Effects on performance in the reaction time task in the various lesion groups: A) reaction times; B) motor times; C) premature responses. All values represent the difference scores between preand postoperative performance. Error bars represent the standard error of the mean. Significant differences are marked with an asterisk $\left(^{*}\right)$, trends with a 'double plus' symbol ( $\left.¥\right)$. 


\section{Reaction times}

Figure 3a shows the reaction times after surgery. Unilateral ischemic damage to the left frontal cortex significantly increased overall reaction time at four weeks after surgery (W4). After right frontal cortex infarction, reaction time also increased but did not reach significance $(p<0.2)$. Reaction time was unaltered after sham-operation. To investigate a possible lateralized difference in reaction time performance between left or right frontal cortical infarction, overall reaction times were subdivided into ipsilesional and contralesional response times. We found such a difference in the right frontal lesion group (Figure 4), where contralesional response times increased immediately after surgery. A significant difference was present at two weeks (W2) after infarction, whereas trends were found for the first couple of days after surgery $(D<4)$, and at weeks 1 and 3 (W1 and W3, respectively).
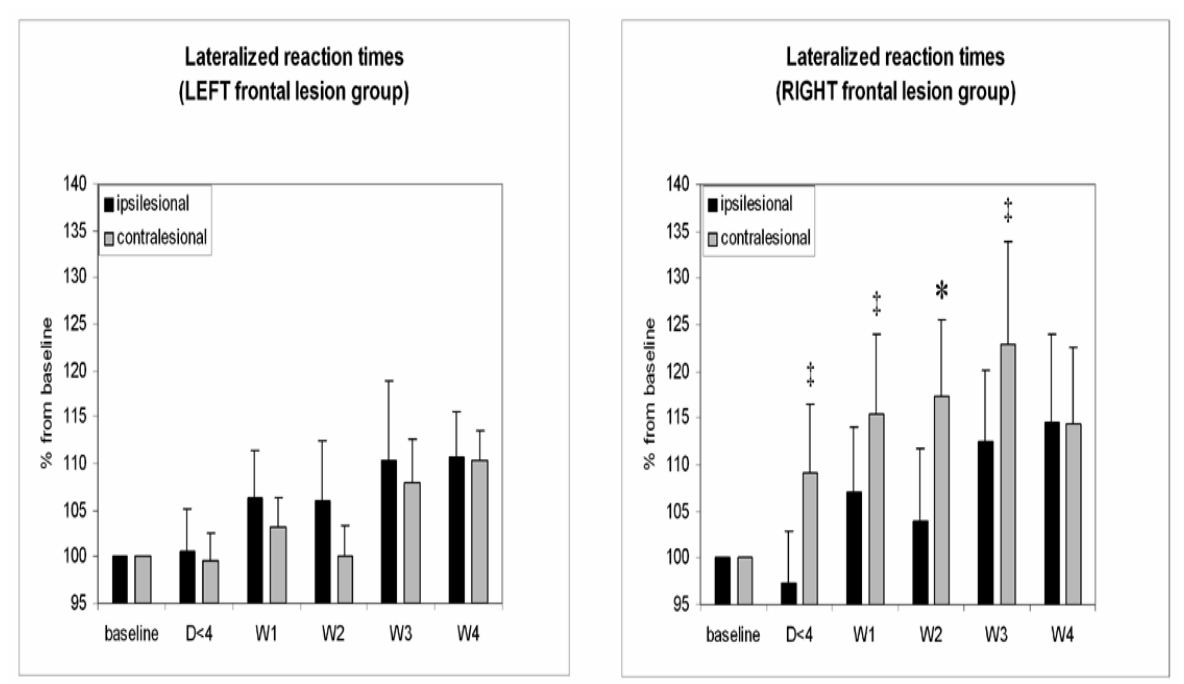

Figure 4. Subdivision of reaction times into ipsilesional and contralesional responses for the left frontal lesion group (A) and the right frontal lesion group (B). All values represent percentage scores from baseline performance, which is set at $100 \%$. Error bars represent the standard error of the mean. Significant differences are marked with an asterisk $\left({ }^{*}\right)$, trends with a 'double plus' symbol ( $\left.¥\right)$. 


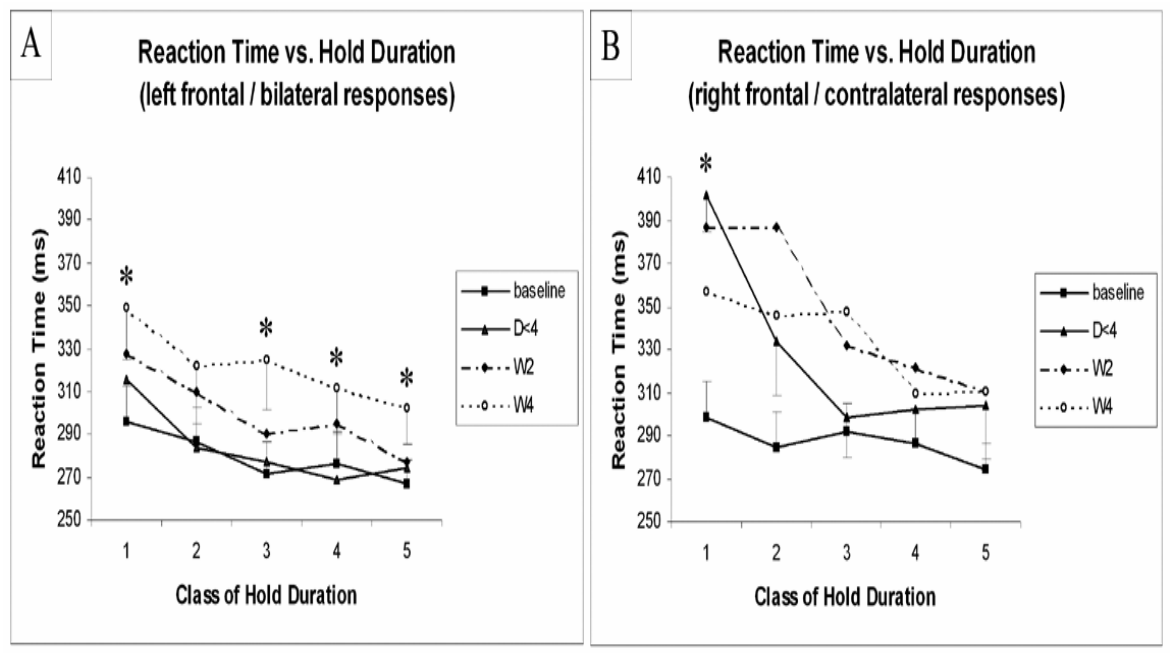

Figure 5. Reaction time performance per class of hold duration. For lesions to the left frontal cortex bilateral responses are plotted against hold duration $(A)$, and for the right frontal lesions the contralateral responses (B). Variable hold durations: Class $1=600 / 700 \mathrm{~ms}$; Class $2=800 / 900 \mathrm{~ms}$; Class $3=1000 / 1100 \mathrm{~ms}$; Class 4 $=1200 / 1300 \mathrm{~ms}$; Class $5=1400 / 1500 \mathrm{~ms}$. Error bars represent the standard error of the mean. Significant differences are marked with an asterisk $\left(^{*}\right)$.

We performed two types of analyses on the reaction time data at $D<4$ and W4: a) reaction time versus hold duration; and b) reaction time distribution. For the gradual reaction time increase within the group of left frontal cortical lesioned animals (Figure $5 \mathrm{~A}$ ), it was found that overall reaction times increased independently of hold duration at W4 (classes 1,3,4 and 5: $p<0.05$; class 2: $p=0.17$ ). Furthermore, significant differences in the distribution of reaction times were found over the total range of reaction times at W4 (classes 1, 2, 3 and 5: $p<0.05$; class 4: $p=0.05$; class 5: $p=0.06$ ). For the lateralized reaction time deficit observed in the group of right frontal cortical lesioned animals (Figure $5 \mathrm{~B}$ ), the increase in contralesional reaction times immediately following surgery was present primarily in trials with short hold durations of $600-900 \mathrm{~ms}$ (at $D<4$ : class $1 p<0.01$, and class $2 p<0.2$; at W4: class $1 p=0.06$, class $2 p=0.09$, and classes 3 and $5 p<0.2$ ). No significant differences were found in the distribution of reaction times at $D<4$ and $W 4$. 


\section{DISCUSSION}

Aiming to model post-stroke slowing of information processing seen in humans, we studied the effects of unilateral infarction of the frontal cortices on reaction time performance in rats. Over the course of four weeks after infarction of the left frontal cortex, reaction times significantly increased whereas motor times decreased. A similar trend was observed after infarction of the right frontal cortex, although not significant. Damage to the frontal cortex thus seems to slow the motor preparatory process, whereas the ability to move was undisturbed. We found the increase in reaction times to be independent of response side or hold duration. In other words, motor readiness to both sides was delayed regardless of the anticipatory time given. This general slowing in reactivity is consistent with the role of the rat frontal cortex in the control of initiation and temporal organization of movement [23]. A further finding was a lateralized reaction time deficit that occurred only after right frontal cortical infarction. The deficit occurred immediately after surgery and only in trials that required a lever press to the contralateral side. Both types of reaction time deficits can also be found in humans. First, a general disturbance in speed of information processing is observed in about $70 \%$ of stroke patients [4-7], particularly after frontal stroke [8, 36]. Second, a lateralized impairment can occur that is called neglect $[37,38]$, and is reflected in a failure to report, respond, or orient to meaningful or novel stimuli contralateral to a cortical lesion [39]. The failure results from an inability to integrate sensorimotor function, and is much more common and severe with right hemisphere than left hemisphere lesions.

In human cognitive experimental psychology the use of response latency as main dependent variable has a long tradition [40]. An important assumption in this respect is that reaction time reflects information processing speed. The type of reaction time testing we employed is similar to what is done in humans, and the nature of responding of rats in this task shows many similarities with that of humans [31]. Thus, reaction time as basic element of information processing is highly comparable between rats and humans. Contrary to most reaction time paradigms in rats that make use of lateralized visual stimulation to elicit a response, we made use of a bivalent auditory stimulus. It is not possible to identify which hemisphere processes the auditory stimulus, but sensory information is generally processed bilaterally. However, the results of the present study are not confounded by a potential bias in auditory discrimination as baseline performance in reaction times did not differ between response side within groups.

Another important issue is that any alterations in sensory capabilities, motor function, or motivation can become confounding factors when measuring cognitive 
performance [41]. Although the lack of testing primary motor and sensory capabilities is a limitation within our study design, we would like to stress the sensitivity of our reaction time paradigm in detecting minute deficits in order of milliseconds; the accuracy of the sampling of events in the Skinner boxes was $1 \mathrm{~ms}$. Moreover, primary motor and sensory capabilities are unlikely to have been affected with lesions being located outside of the sensorimotor cortex (see Figure 2). The observed decreases in motor times indicate that the frontal lesions did not affect the execution of movement. Although this might be explained as an epiphenomenon or an effect of repeated training, we believe it to be compensatory to the increase in reaction time: when maximally prepared, impairment of the motor initiation process may result in increased motor execution. Furthermore, since the proportion of premature responses hardly changed after frontal cortex infarction, the increase in reaction times is not likely to be caused by a decrease in response inhibition.

What could be the nature of the observed reaction time deficits? Theoretically, deficits in reactivity can be caused by a disability to initiate a motor response or by a disruption of attentional processes, or both. In general, a hold duration-dependent increase in reaction time is interpreted as a disability to prepare the initiation of a motor response [10, 20], whereas an increase in the variation of reaction time distribution has been associated with a decrease in attentional processes related to the preparation of movement [34, 35]. As our analysis of reaction time distribution shows, a disturbance of attentional function could have caused the general slowing in reactivity after left frontal infarction. On the other hand, the lateralized impairment observed immediately after right frontal infarction, may be related to a disability in preparing movement initiation under the pressure of time.

How can these reaction time deficits be explained? The origin of the lateralized reaction time deficit almost definitely lies within the frontal cortex, since it emerged immediately following surgery. By contrast, the general slowing in reactivity may be the result of either degenerative or plastic changes within the cerebral cortex or subcortical areas. The frontal lobes have been shown to play a critical role in intentional (or motor) systems $[42,43]$. Unilateral lesions of the frontal cortices are known to cause a contralateral 'neglect' in both rats and primates. This inability to respond to stimuli in contralateral space can be interpreted as either reflecting attentional or representational neglect [42] or, alternatively, an intentional or 'output' form of neglect [43]. A putative circuit for directing spatial attention has been identified [44, 45], which is equivalent to the distributed cortical attentional system proposed in primates [46]. The circuit in the rat is thought to involve medial agranular cortex (Fr2), ventrolateral orbital (Fr1) and posterior parietal cortex (Par1). In addition, changes in neurotransmitter function might explain both the biochemical and behavioural findings observed 
after cortical lesions [26, 47, 48]. A recent report covering 20 years of research using a rat model of contralateral neglect [49], explains the neurobiology of this phenomenon as an acute loss of dopaminergic input to the dorsocentral striatum showing recovery with dopaminergic agonists. The lateralized deficit in movement initiation we observed may thus be caused by dysfunction of the dopaminergic system. Furthermore, the general slowing in reactivity might be related to degeneration and subsequent plastic changes within the basal forebrain cholinergic system, which is known to influence attentional function and optimize cortical information processing [50].

In conclusion, two types of reaction time deficits were observed after unilateral infarction to the frontal cortices in rats. Infarction to the left frontal cortex of rats induced a general slowing in reactivity similar to the mental slowing observed in stroke patients, whereas a lateralized deficit showing right hemispheric dominance was observed that resembles left-sided neglect in humans. We hypothesize the former may be related to a decrease in either attentional function or speed of information processing following plastic changes within the cholinergic basal forebrain system, whereas the latter may be due to impairment of motor initiation caused by an immediate loss of dopaminergic input to the dorsocentral striatum. Measuring reaction time performance after cortical infarction in rats could offer a useful tool to model human post-stroke cognitive impairment. 


\section{REFERENCES}

1. Zilles K, Wree A. Cortex: areal and laminar structure. In: Paxinos G, editor. The Rat Nervous System. San Diego: Academic Press; 1995. p. 649-85.

2. Paxinos G, Watson C. The Rat Brain in Stereotaxic Coordinates. 4th edition ed. New York: Academic Press; 1998.

3. O'Brien JT, Erkinjuntti T, Reisberg B, Roman G, Sawada T, Pantoni L, et al. Vascular cognitive impairment. Lancet Neurol. 2003 Feb;2(2):89-98.

4. Hochstenbach J, Mulder T, van Limbeek J, Donders R, Schoonderwaldt H. Cognitive decline following stroke: a comprehensive study of cognitive decline following stroke. J Clin Exp Neuropsychol. 1998;20(4):503-17.

5. Loranger M, Lussier J, Pepin M, Hopps SL, Senecal B. Information-processing speed and assessment of early response latency among stroke patients. Psychol Rep. 2000 Dec;87(3 Pt 1):893-900.

6. Gerritsen MJ, Berg IJ, Deelman BG, Visser-Keizer AC, Meyboom-de Jong B. Speed of information processing after unilateral stroke. J Clin Exp Neuropsychol. 2003 Feb;25(1):1-13.

7. Rasquin SM, Lodder J, Ponds RW, Winkens I, Jolles J, Verhey FR. Cognitive functioning after stroke: a one-year follow-up study. Dement Geriatr Cogn Disord. 2004;18(2):138-44.

8. Sachdev PS, Brodaty H, Valenzuela MJ, Lorentz L, Looi JC, Wen W, et al. The neuropsychological profile of vascular cognitive impairment in stroke and TIA patients. Neurology. 2004 Mar 23;62(6):912-9.

9. Steckler T, Muir JL. Measurement of cognitive function: relating rodent performance with human minds. Brain Res Cogn Brain Res. 1996 Jun;3(3-4):299-308.

10. Hauber W, Bubser M, Schmidt WJ. 6-Hydroxydopamine lesion of the rat prefrontal cortex impairs motor initiation but not motor execution. Exp Brain Res. 1994;99(3):524-8.

11. Sakurai $Y$, Shibata R, Kurachi M. Effect of 6-hydroxydopamine lesions of the medial prefrontal cortex of the rat on performance in a reaction time task. Eur Neuropsychopharmacol. 1995 Mar;5(1):69-73.

12. Baunez C, Salin P, Nieoullon A, Amalric M. Impaired performance in a conditioned reaction time task after thermocoagulatory lesions of the fronto-parietal cortex in rats. Cereb Cortex. 1998 Jun;8(4):301-9.

13. Ward NM, Sharkey J, Marston HM, Brown VJ. Simple and choice reaction-time performance following occlusion of the anterior cerebral arteries in the rat. Exp Brain Res. 1998 Dec;123(3):269-81.

14. Broersen LM, Uylings HB. Visual attention task performance in Wistar and Lister hooded rats: response inhibition deficits after medial prefrontal cortex lesions. Neuroscience. 1999;94(1):47-57.

15. Blokland A, Broersen LM, Uylings HB, Jolles J. Intact spatial discrimination performance but impaired reaction time performance after frontal cortex lesions in the rat. Neurosci Res Comm. 2003;33(1):1-16.

16. Chudasama Y, Passetti F, Rhodes SE, Lopian D, Desai A, Robbins TW. Dissociable aspects of performance on the 5-choice serial reaction time task following lesions of the dorsal anterior cingulate, infralimbic and orbitofrontal cortex in the rat: differential effects on selectivity, impulsivity and compulsivity. Behav Brain Res. 2003 Nov 30;146(1-2):105-19.

17. Risterucci C, Terramorsi D, Nieoullon A, Amalric M. Excitotoxic lesions of the prelimbic-infralimbic areas of the rodent prefrontal cortex disrupt motor preparatory processes. Eur J Neurosci. 2003 Apr;17(7):1498-508.

18. Christakou A, Robbins TW, Everitt BJ. Prefrontal cortical-ventral striatal interactions involved in affective modulation of attentional performance: implications for corticostriatal circuit function. J Neurosci. 2004 Jan 28;24(4):773-80.

19. Schallert T, Hernandez TD, Barth TM. Recovery of function after brain damage: severe and chronic disruption by diazepam. Brain Res. 1986 Jul 30;379(1):104-11.

20. Brown VJ, Bowman EM, Robbins TW. Response-related deficits following unilateral lesions of the medial agranular cortex of the rat. Behav Neurosci. 1991 Aug;105(4):567-78. 
21. Muir JL, Everitt BJ, Robbins TW. The cerebral cortex of the rat and visual attentional function: dissociable effects of mediofrontal, cingulate, anterior dorsolateral, and parietal cortex lesions on a five-choice serial reaction time task. Cereb Cortex. 1996 May-Jun;6(3):470-81.

22. Brasted PJ, Dunnett SB, Robbins TW. Unilateral lesions of the medial agranular cortex impair responding on a lateralised reaction time task. Behav Brain Res. 2000 Jun 15;111(1-2):139-51.

23. Kolb B. Functions of the frontal cortex of the rat: a comparative review. Brain Res. 1984 Nov;320(1):6598.

24. Fuster JM. Executive frontal functions. Exp Brain Res. 2000 Jul;133(1):66-70.

25. Warren JM. Functional lateralization of the brain. Ann N Y Acad Sci. 1977 Sep 30;299:273-80.

26. Robinson RG. Differential behavioral and biochemical effects of right and left hemispheric cerebral infarction in the rat. Science. 1979;205(4407):707-10.

27. Walker SF. Lateralization of functions in the vertebrate brain: a review. $\mathrm{Br} \mathrm{J}$ Psychol. 1980 Aug;71(3):329-67.

28. Bianki VL. Lateralization of functions in the animal brain. Int J Neurosci. 1981;15(1-2):37-47.

29. Denenberg VH. Lateralization of function in rats. Am J Physiol. 1983 Oct;245(4):R505-9.

30. Carlson JN, Glick SD. Cerebral lateralization as a source of interindividual differences in behavior. Experientia. 1989 Sep 15;45(9):788-98.

31. Blokland A. Reaction time responding in rats. Neurosci Biobehav Rev. 1998 Oct;22(6):847-64.

32. Watson BD, Dietrich WD, Busto R, Wachtel MS, Ginsberg MD. Induction of reproducible brain infarction by photochemically initiated thrombosis. Ann Neurol. 1985;17(5):497-504.

33. Hoff El, Oude Egbrink MG, Heijnen VV, Steinbusch HW, van Oostenbrugge RJ. In vivo visualization of vascular leakage in photochemically induced cortical infarction. J Neurosci Methods. 2005 Jan 30;141(1):135-41.

34. Pribram KH, McGuinness D. Arousal, activation, and effort in the control of attention. Psychol Rev. 1975 Mar;82(2):116-49.

35. Blokland A, Honig W. Intra-striatal haloperidol and scopolamine injections: effects on choice reaction time performance in rats. Eur Neuropsychopharmacol. 1999 Dec;9(6):523-31.

36. Leskela M, Hietanen M, Kalska H, Ylikoski R, Pohjasvaara T, Mantyla R, et al. Executive functions and speed of mental processing in elderly patients with frontal or nonfrontal ischemic stroke. Eur J Neurol. 1999;6(6):653-61.

37. Samuelsson H, Hjelmquist EK, Jensen C, Ekholm S, Blomstrand C. Nonlateralized attentional deficits: an important component behind persisting visuospatial neglect? J Clin Exp Neuropsychol. 1998 Feb;20(1):73-88.

38. Schendel KL, Robertson LC. Using reaction time to assess patients with unilateral neglect and extinction. J Clin Exp Neuropsychol. 2002 Oct;24(7):941-50.

39. Heilman KM. Neglect and Related Disorders. In: Heilman KM, Valenstein E, editors. Clinical Neuropsychology. 4 ed. Oxford: Oxford University Press; 2003. p. 296-346.

40. Pachela RG. The interpretation of reaction time in information-processing research. In: Kantowitz $\mathrm{BH}$, editor. Human information processing - Tutorials in performance and cognition. Hillsdale: Lawrence Erlbaum Associates; 1974. p. 41-82.

41. DeVries AC, Nelson RJ, Traystman RJ, Hurn PD. Cognitive and behavioral assessment in experimental stroke research: will it prove useful? Neurosci Biobehav Rev. 2001 Jun;25(4):325-42.

42. Heilman KM, Valenstein E. Frontal lobe neglect in man. Neurology. 1972 Jun;22(6):660-4.

43. Watson RT, Miller BD, Heilman KM. Nonsensory neglect. Ann Neurol. 1978 Jun;3(6):505-8.

44. King VR, Corwin JV. Comparisons of hemi-inattention produced by unilateral lesions of the posterior parietal cortex or medial agranular prefrontal cortex in rats: neglect, extinction, and the role of stimulus distance. Behav Brain Res. 1993 Apr 30;54(2):117-31. 
45. King VR, Corwin JV. Spatial deficits and hemispheric asymmetries in the rat following unilateral and bilateral lesions of posterior parietal or medial agranular cortex. Behav Brain Res. 1992 Sep 28;50(12):53-68.

46. Mesulam MM. A cortical network for directed attention and unilateral neglect. Ann Neurol. 1981 Oct;10(4):309-25.

47. van Vleet TM, Heldt SA, Corwin JV, Reep RL. Infusion of apomorphine into the dorsocentral striatum produces acute drug-induced recovery from neglect produced by unilateral medial agranular cortex lesions in rats. Behav Brain Res. 2003 Aug 14;143(2):147-57.

48. Pearlson GD, Kubos KL, Robinson RG. Effect of anterior-posterior lesion location on the asymmetrical behavioral and biochemical response to cortical suction ablations in the rat. Brain Res. 1984;293(2):241-50.

49. Reep RL, Corwin JV, Cheatwood JL, Van Vleet TM, Heilman KM, Watson RT. A rodent model for investigating the neurobiology of contralateral neglect. Cogn Behav Neurol. 2004 Dec;17(4):191-4.

50. Baxter MG, Chiba AA. Cognitive functions of the basal forebrain. Curr Opin Neurobiol. 1999 Apr;9(2):178-83. 



\section{Effects of right hemisphere cortical infarction and muscarinic}

acetylcholine receptor blockade on spatial visual attention performance in rats

Erik I. Hoff

Robert J. van Oostenbrugge

Marjolein Liedenbaum

Harry W.M. Steinbusch

Arjan Blokland 


\section{ABSTRACT}

The syndrome of hemispatial neglect is defined as an inability to report, respond or orient to stimuli contralateral to a cerebral lesion despite intact elementary sensory or motor function. This syndrome is typically observed after lesions of the right cerebral cortex, and has been associated with impairment of attention. We studied whether visual attention performance is impaired after right hemisphere infarction in rats. Using a behavioural paradigm measuring spatial visual attention, we tested the effects of photothrombotic infarction to either the frontal cortex or the parietal cortex on attention performance. Since the cholinergic system is known to modulate attention performance, we additionally evaluated the role of cholinergic receptor blockade with scopolamine in our task paradigm. Our results show a transient response bias immediately after cortical infarction, with a decrease in contralesional responses and an increase in contralesional omissions after frontal infarction. Parietal infarction and systemic administration of scopolamine also resulted in a decrease in correct responses and an increase in omissions, but without a difference in side responding. In conclusion, right frontal infarction induces a transient impairment in contralesional spatial visual attention that we explain as left-sided neglect. Right parietal infarction and cholinergic blockade shows non-lateralized deficits in spatial visual attention, suggestive of global attentional impairment. We postulate that both effects of cortical infarction on attention performance may be related to cholinergic dysfunction. Our study confirms the role of frontal and parietal cortices in attention performance in rats, and corroborates the theory that attention performance is impaired in hemispatial neglect in human stroke patients. 


\section{INTRODUCTION}

The syndrome of hemispatial neglect is defined as an inability to report, respond or orient to stimuli contralateral to a cerebral lesion despite intact elementary sensory or motor function [3]. This syndrome is typically observed in stroke patients, and is more common and severe after lesions in the right hemisphere compared to the left hemisphere. The inability to respond most likely originates from a failure to integrate sensorimotor function. Phenomena associated with the neglect syndrome can be subdivided into three major categories: representational deficits, action-intentional disorders ('motor neglect') and hemi-inattention ('sensory neglect') [4]. Using a reaction time task, we previously compared the effects of left frontal cortex infarction to right frontal cortex infarction in rats [5]. We observed a form of motor neglect that occurred only after right frontal cortical infarction. However, fundamental to cognitive information processing are mechanisms of attention. Disturbances in attention have been associated with the neglect syndrome in humans [6].

Mesulam introduced the concept of the right hemisphere cortical attentional system [7], which may explain the occurrence of attention deficits in the neglect syndrome. Herein, the right cerebral hemisphere is considered dominant for spatial visual attention through a cortical network that surveys both sides of personal and extrapersonal space. This neuronal network allows for the narrowing of the attentional focus on motivationally relevant spatial stimuli. Additionally, the right hemisphere is involved in non-spatially sustained attention [8], which has also been shown to be impaired in hemispatial neglect [9-11]. Right frontal and parietal cortical regions appear particularly important for maintaining spatial attention in humans [12]. A putative circuit for directing spatial attention has also been identified in rodents, involving the medial frontal cortex, posterior parietal cortex and dorsolateral striatum [13-15].

Lateralized deficits in visual attention performance may alternatively be caused by dysfunction of neurotransmitter-specific networks [16, 17]. The basal forebrain cholinergic system is known to modulate attention performance by optimizing cortical information processing [18-20]. Several studies have implicated cholinergic projections to the cortex in visual attention performance in the rat [21-27]. Interestingly, lateralized attentional functions of these cortical cholinergic inputs have been described following unilateral damage to their cells of origin within the basal forebrain $[17,28]$. It is hypothesized that right-hemisphere cortical inputs mediate signal detection processes of right-hemisphere attention systems, whereas left-hemisphere cortical inputs are involved in executive aspects of attention performance by lefthemisphere circuits. Thus, loss of right-hemisphere cholinergic function may explain the occurrence of left-sided visual spatial neglect. 
As mentioned above, hemispatial neglect originates from a failure to integrate sensorimotor function along a spectrum of motor neglect and sensory neglect. The present study investigates whether impairment of attention performance occurs after right hemisphere infarction in rats. We aimed to determine the contribution of a possible attentional impairment to the neglect phenomenon observed previously after right frontal cortical infarction [5]. Using a behavioural paradigm measuring spatial visual attention, we tested the effects of infarction to either the frontal cortex or the parietal cortex of the right hemisphere. Furthermore, to evaluate the role of cholinergic function on attention performance in the behavioural paradigm, we tested spatial visual attention in a group of non-lesioned animals with and without administration of scopolamine, a muscarinic acetylcholine receptor antagonist.

\section{MATERIALS AND METHODS}

\section{Subjects}

All experimental procedures were approved by the local ethical committee for animal experiments at Maastricht University and met governmental guidelines. Forty male Lewis rats served as subjects in this study. They were housed individually in standard type III Makrolon cages, on sawdust bedding in an air-conditioned room of about $21^{\circ} \mathrm{C}$ with $45-55 \%$ humidity under a reversed light/dark cycle (lights on from 7 p.m. to 7 a.m.) and had ad libitum access to water. To prevent a motivational bias during behavioural testing, the rats were food-restricted and given 12-14 g laboratory chow per day. In the weekends, the rats had ad libitum access to food up until 12 hours prior to testing. In doing so, the weight of the animals was kept at $85 \%$ of a freely-fed reference group.

\section{Experimental design}

Rats were trained in a behavioural paradigm measuring spatial visual attention, as described previously [29]. We performed two types of experiments. One experiment tested attention performance after frontal cortex infarction $(n=10)$, parietal cortex infarction $(n=10)$, and in sham-operated animals $(n=10)$ (Experiment I). Since neglect phenomena are primarily observed after right-sided brain damage in humans, lesions were induced in the right hemisphere. Another experiment tested the effects of muscarinic acetylcholine receptor blockade on task performance in a group of nonlesioned animals $(n=10)$ (Experiment II). These rats were tested after intraperitoneal 
administration of scopolamine hydrochloride (Sigma Aldrich) $0.1 \mathrm{mg} / \mathrm{kg}, 0.3 \mathrm{mg} / \mathrm{kg}$ or vehicle (sterile saline).

\section{Behavioural procedures}

\section{Apparatus}

Spatial visual attention performance was trained and tested in ten identical Skinner boxes $(40 \times 30 \times 33 \mathrm{~cm})$. The ceiling of these conditioning chambers contained a light that illuminated the conditioning chamber during experiments. The left and right sidewalls served as control panels. A recess $(5 \times 5 \mathrm{~cm})$, built into the left side panel $2.5 \mathrm{~cm}$ above the grid floor, contained a food tray into which a pellet dispenser delivered 45-mg food pellets (Bioserve, Frenchtown, NJ, USA). Two retractable stainless steel levers ( $4 \mathrm{~cm}$ wide) projected $2 \mathrm{~cm}$ into the conditioning chamber and were located $6 \mathrm{~cm}$ from both sides of the recess, $12 \mathrm{~cm}$ above the grid floor. The conditioning chambers were enclosed in sound-attenuating housing. Background noise was produced by a radio and an exhaust fan. A personal computer controlled the experimental equipment and collected the data.

\section{Spatial visual attention task}

The rats first underwent a magazine training. In this training about 30 food pellets were randomly supplied during a period of 15 minutes; all food pellets were consumed. The levers were retracted from the operant conditioning chamber during this phase. After completion of this training, the rats underwent a Continuous Reinforcement (CRF) training schedule. In this CRF training, either the right or left lever was presented at random, but both with the same number of times per session. As soon as the rat pressed a lever, it was retracted and a food pellet was delivered. After the rat had taken the food pellet from the tray, there was an inter-trial interval of $5 \mathrm{sec}$ onds. Then the next trial was started by insertion of a lever into the Skinner box. A total of 80 food pellets could be obtained in a session lasting a maximum of $40 \mathrm{~min}$. Generally, the rats completed the session within $20 \mathrm{~min}$, in which all food pellets were consumed. Qualitative observations indicated that the rats were facing the front of the Skinner box wall with their nose headed towards the centre of the wall each time a trial started. Previous studies have shown that the rats only miss one or two trials per session, indicating that they are actively engaged in the task [29, 30]. 

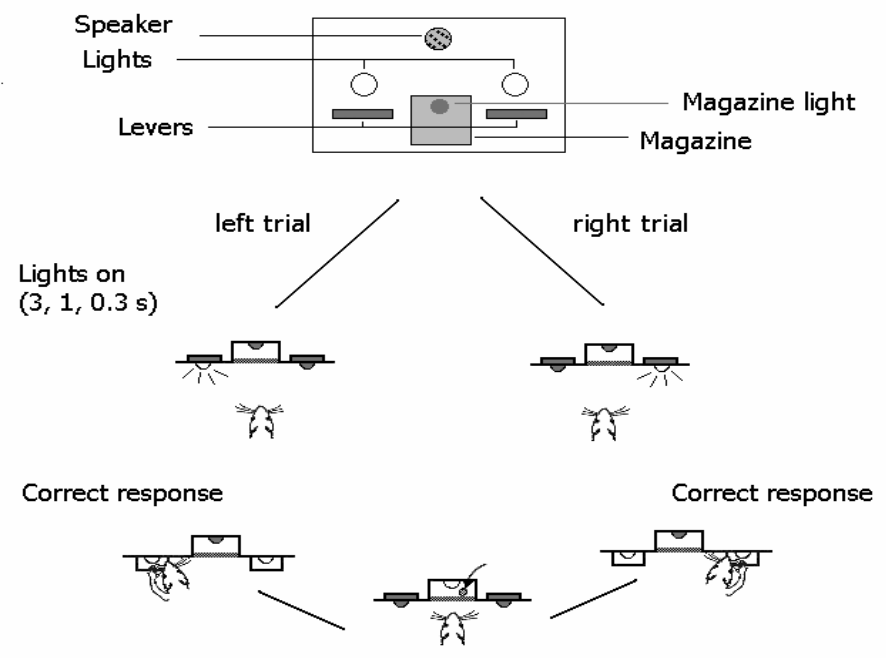

Response on non-signalled side $=$ error (no reward)

Figure 1. Schematic representation of the behavioural paradigm, showing the operating wall of the Skinner box (top) and the series of stages in the spatial visual attention task.

In the behavioural test paradigm (Figure 1), a light stimulus was presented either on the left side or on the right side of the food reward tray. The duration of the light stimulus varied randomly between 3,1 and 0.3 seconds. One second after the light stimulus was extinguished, the two levers were inserted simultaneously. When the rat hit the lever on the side of the prior light stimulus (correct response), the rat was rewarded with a food pellet followed by an inter trial interval (ITI) of 5 to 10 seconds. When the rat hit the lever on the opposite side of the previous light stimulus (incorrect response), the rat was not rewarded. After a time-out period of 5 seconds was followed by an ITI. When the rat did not hit a lever within 3 seconds (omission), the rat was not rewarded and both levers were retracted followed by a time-out period of 5 seconds and an ITI. A total of 80 food pellets could be obtained during a session lasting a maximum of $20 \mathrm{~min}$. 
The main behavioural measures derived from this task were as follows:

1) Index $Y$ : a signal detection measure for response bias. It was calculated as the absolute value of $\frac{\text { Percentage correct left }- \text { Percentage correct right }}{\text { Percentage correct left }+ \text { Percentage correct right }}$

This measure determined whether the rats develop a preference to respond to one side. A value of zero would indicate that there was no side bias, whereas a value of 1 indicated that the rats respond to one side only [31]. With absolute values between 0 and 1 , index $Y$ does not discriminate between left-sided and right-sided response biases.

2) Percentage Correct: $\frac{\text { Correct responses }}{\text { Total number of trials - Omissions }}$

To detect response side bias, the percentage of correct responses was subdivided into right-sided and left-sided responses.

3) Omissions: total number of times the rat did not hit either lever within the required time. To detect response side bias, the number of omissions was subdi-i vided into right-sided and left-sided response failures.

Rats were trained in the behavioural paradigm with two sessions daily until stable performance was achieved (no improvement on five successive training sessions).

\section{Experiment I - visual attention after cortical infarction}

Body weight and task performance were matched between experimental groups prior to surgery. Anaesthesia was induced by inhalation of halothane $4 \%$ and maintained with halothane $1 \%$ and a mixture of $\mathrm{O} 2 / \mathrm{N} 2 \mathrm{O}(1: 2)$ in spontaneously breathing rats. As described previously [32], cortical infarction was induced by photochemically initiated thrombosis through intravenous injection of the light-sensitive dye Erythrosin $B$ (Sigma-Aldrich, Zwijndrecht, The Netherlands) and illumination of the skull with a monochromatic light source (DPSS laser, Uniphase, Germany). The light source, with a diameter of $2.4 \mathrm{~mm}$, was positioned perpendicular to the skull surface in the centre of a perforated filtration paper that absorbed any leakage of blood; its beam was positioned at $2.7 \mathrm{~mm}$ anterior and $2.7 \mathrm{~mm}$ lateral to Bregma's point for infarction of the frontal cortex, and at $2.7 \mathrm{~mm}$ posterior and $5.4 \mathrm{~mm}$ lateral to Bregma's point for infarction of the parietal cortex. In sham-operated animals, the order of dye injection and illumination was reversed. 
We found the animals to behave normally already a few hours after cortical infarction: grooming, gross motor function, eating, and drinking. From the first day after surgery, the rats were able to respond in the Skinner box task and to complete the training session (80 trials within $20 \mathrm{~min}$ ), which was similar to the performance prior to surgery. Thus, no recovery period was needed after surgery and rats were trained and tested continuously twice daily on three alternate weekdays for three weeks.

At the end of behavioural testing, histology was performed on all rat brains in order to verify lesion volume and location. Around the lesion site, the brain was serially cut into $30-\mu \mathrm{m}$ coronal sections and mounted onto slides. For the quantification of infarct volumes, every fifth section was stained using a May-Grünwald-Giemsa protocol [33]. The area and depth of the lesion were delineated in each section on a stereological microscopy set-up. Lesion volume (in $\mathrm{mm} 3$ ) was calculated by multiplying lesion area by intersection thickness.

\section{Experiment II - visual attention after cholinergic blockade}

Task performance was tested during two sessions on the same day 30 minutes after intraperitoneal injection of either vehicle solution ( $1 \mathrm{ml}$ of sterile saline) or scopolamine $0.1 \mathrm{mg} / \mathrm{kg}$ dissolved in $1 \mathrm{ml}$ vehicle solution. Two days later, task performance was re-tested 30 minutes after injection of either $1 \mathrm{ml}$ vehicle solution or scopolamine $0.3 \mathrm{mg} / \mathrm{kg}$ dissolved in $1 \mathrm{ml}$ vehicle solution. The data after vehicle injection were averaged to serve as control (baseline data).

\section{Data processing and statistical analyses}

All statistical analyses were done using SPSS software (version 12). Group differences in lesion volume and body weight were compared using Student's t-testing (two-tailed, independent samples). In Experiment I, baseline-data were obtained by averaging the last four test sessions prior to the induction of cortical infarction. Follow-up data were averaged across two sessions acquired on days 1 and 3 , and if a weekend had passed on days 1 and 4 after surgery (D4). In the weeks following surgery, data were averaged of days 6 and 8 (W1), days 13 and 15 (W2), and days 20 and 22 (W3). Data of Experiment I were analyzed using a three-way ANOVA (Lesion group as between subjects factor; Time and Side of response as within subjects factors). Differences between conditions were evaluated using an LSD post hoc test. To test for lateralized responding, ipsilesional performance was deducted from contralesional performance and subjected to a one-sample ANOVA. In Experiment II, vehicle injection data were averaged to serve as baseline. The effects of scopola- 
mine were tested using a two-way ANOVA (Dose and Stimulus duration as within subjects factor). To evaluate possible side differences in responding, we evaluated left and right sided responses at the shortest stimulus duration using a one-way ANOVA (Side of response as within subjects factor).

\section{RESULTS}

\section{Experiment I - visual attention after cortical infarction}

\section{Lesion group characteristics}

Figure 2 shows the location and volume of cortical infarction. Parietal lesions were located in the primary sensorimotor cortex (Par1), also known as posterior parietal cortex. Frontal lesions were located in the rostral part of the cortical premotor areas Fr1 and Fr2, respectively known as the lateral frontal cortex and medial frontal cortex. Five animals were excluded from statistical analysis.
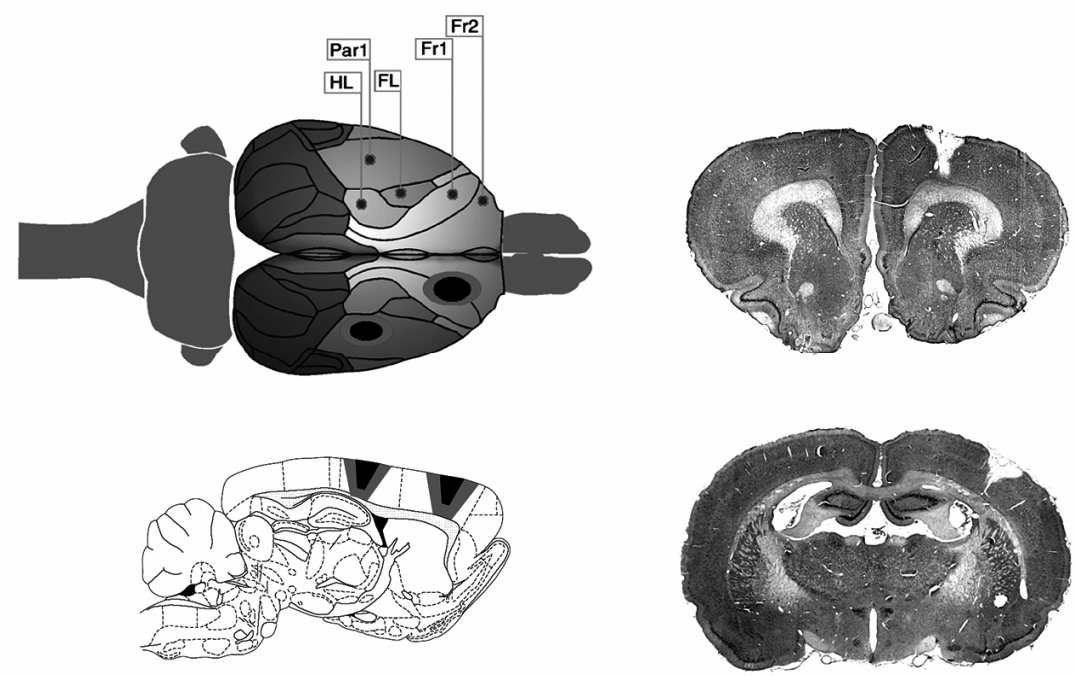

Figure 2. On left: dorsal and sagittal view of the rat brain showing the variation in lesion extent (grey circle) and the greatest common divisor of all lesions within an experimental group (black circle). On right: coronal sections with May-Grünwald-Giemsa staining showing phototrombotic infarction to the frontal and parietal cortices. Frontal region: Fr $1=$ frontal area 1 (or primary motor cortex), Fr2 = frontal area 2 (also known as medial agranular cortex). Parietal region: $\mathrm{FL}=$ sensorimotor area of forelimb, $\mathrm{HL}$ = sensorimotor area of hind limb, Par1 = parietal area 1 (or primary somatosensory cortex). The dorsal view was adopted from Zilles and Wree [1], the sagittal view from Paxinos and Watson [2]. 
One animal was unable to master the behavioural task adequately, two animals died during surgical procedures (anaesthesia), and another two animals were excluded from analysis because at post-mortem examination of the brain no lesion could be detected. Therefore, the final number of animals group was: right frontal cortex $(n=9)$, right parietal cortex $(n=7)$, and sham-operated $(n=9)$. Mean lesion volume at the end of behavioural testing was greater for right frontal infarction $(1.76 \pm 0.24 \mathrm{~mm} 3)$ as compared with right parietal infarction $(1.22 \pm 0.20 \mathrm{~mm} 3)$ of the cerebral cortex $(t(14)=2.93, p<0.05)$. No lesions were detected in sham-operated animals. No differences in body weight were found between groups during the four weeks of behavioural testing after infarction.

\section{Spatial visual attention after cortical infarction}

Figures 3 and 4 summarize the results after cortical infarction. At the longer stimulus durations of 1 and 3 seconds, no group differences were observed for the behavioural parameters (all F's $<1.3$, ns). Consequently, we decided to only evaluate lesion effects at the shortest stimulus duration of $300 \mathrm{~ms}$. This dependency on stimulus duration indicates that attention performance was related to task difficulty and attentional load rather than an inability to complete the task. Changes in spatial visual attention were observed only within the lesion groups and not after sham operation, which shows that performance was not disturbed by the surgical procedures, in particular anaesthesia. Inspection of the data revealed that effects occurred only in the first days after cortical infarction (D4) and had disappeared after one week (W1). Therefore, we evaluated lesion effects by analyzing performance for the first three time points (Baseline, D4 and W1).
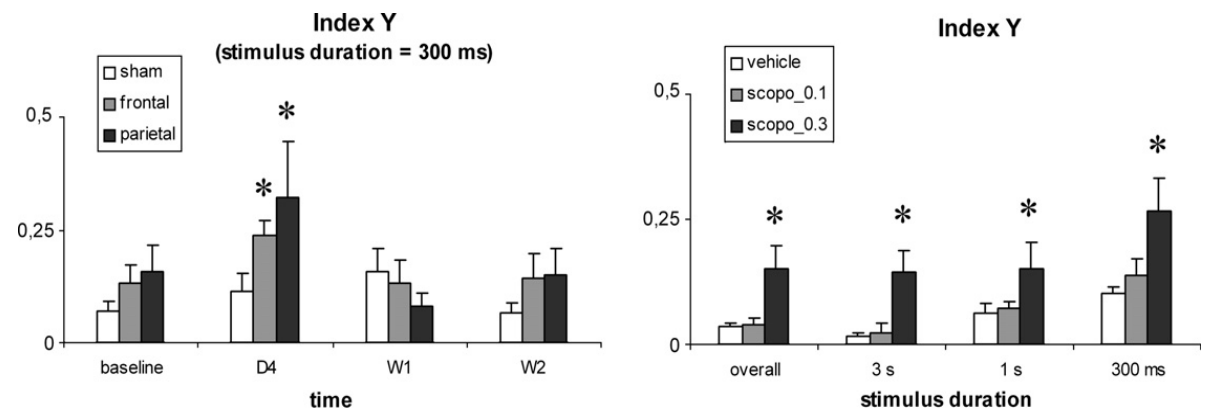

Figure 3. Response side bias in the spatial visual attention task. Left graph shows index $Y$ in Experiment I for the shortest stimulus duration. Right graph shows index $Y$ in Experiment II. Error bars represent the standard error of the mean. Post hoc analyses: $\left(^{*}\right)$ significant difference between groups. 

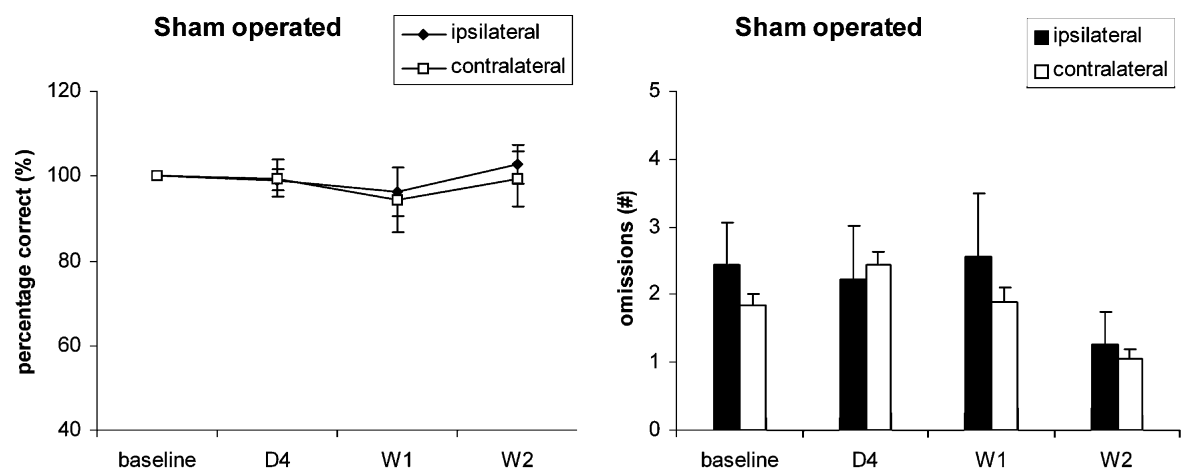

Frontal lesion

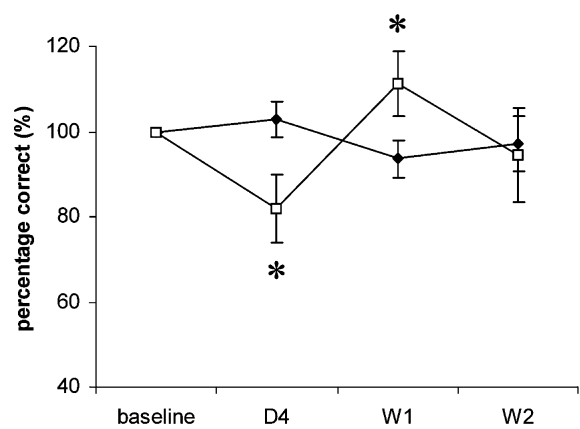

Frontal lesion

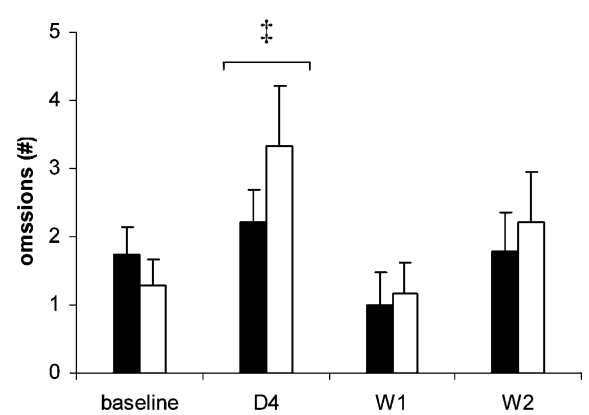

Parietal lesion

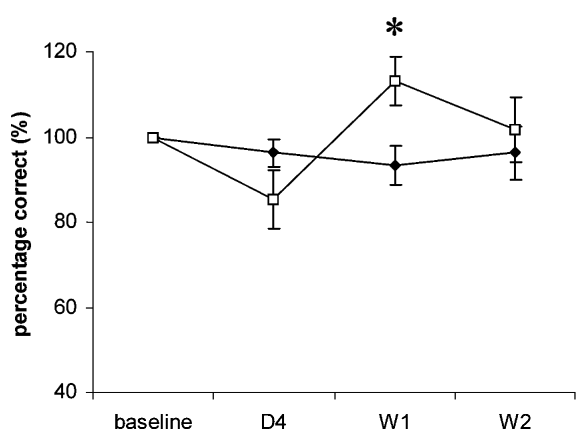

Parietal lesion

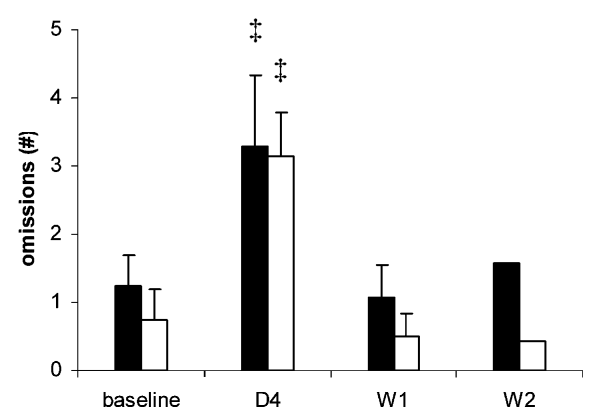

Figure 4. Percentage correct responses and number of omissions subdivided into ipsilateral and contralateral side responses for sham-operated animals, right frontal lesion group, and right parietal lesion group. For the percentage of correct responses, all values represent percentage scores from baseline performance (set at $100 \%$ ). The number of omissions is an absolute value. Error bars represent the standard error of the mean. Post hoc analyses: $\left(^{*}\right)$ significant difference between groups $(p<0.05) ;(\ddagger)$ significant difference within groups $(p<0.05)$. 
For index $Y$, we found a clear effect of Time $(F(2,44)=9.48, p<0.01)$ that tended to be different for the lesion groups $(F(2,44)=2.29, p<0.08)$. Evaluation of the effect of Time within each group showed that index $Y$ was affected after frontal $(F(2,16)=3.75$, $p<0.05)$ and parietal infarction $(F(2,12)=5.64, p<0.05)$ and not after sham operation $(F(2,16)=0.08, n s)$. Post hoc analysis revealed that both lesion groups differed from the sham group at D4, and the lesion effect was similar after frontal and parietal infarction.

For Percentage Correct, a Lesion by Time by Side interaction effect was found $(F(4,44)=3.80, p<0.05$; see Figure 4). Analysis within groups did not reveal Time, Side, and Time by Side interaction effects in the sham group (all F's $<1.67$, ns), indicating that surgery did not affect the performance of these animals. Clear Time by Side interaction effects were found in the frontal lesion group $(F(2,16)=17.41, p<0.01)$ as well as the parietal lesion group $(F(2,14)=7.33, p<0.01)$. Inspection of Figure 4 shows that these effects were related to changes in performance contralateral to the lesion whereas ipsilateral performance was unaltered. Post hoc analysis showed that contralateral performance did not change after sham operation, and differed significantly at D4 and W1 for the frontal lesion group and at W1 for the parietal lesion group.

For the measure Omissions, a clear effect of Time was found $(F(2,44)=9.48$, $p<0.01)$. Within group analysis showed that there was no effect of Time in the sham group $(F(2,16)=1.06$, ns), whereas Time effects were present in the frontal $(F(2,16)=3.75, p<0.05)$ and parietal groups $(F(2,12)=5.64, p<0.05)$. Furthermore, a marginal Time by Side interaction effect was observed $(F(4,44)=2.29, p<0.07)$. Analysis within groups showed that the Side by Time interaction effect occurred only in the frontal lesion group $(F(2,16=4.14, p<0.05)$, and not in the parietal lesion and sham operation group ( $\mathrm{F}$ 's $<1, \mathrm{~ns})$.

\section{Experiment II - visual attention after cholinergic blockade}

Figures 3 and 5 show spatial visual attention task performance with or without administration of scopolamine. The measure index $Y$ for side bias increased with dose of scopolamine $(F(2,27)=5.49, \quad p<0.05)$ and shorter stimulus durations $(F(2,54)=17.77, p<0.01)$. The effect of scopolamine was independent of stimulus duration $(F(4,54)=0.74$, ns $)$. A similar result was found for the percentage of correct responses as we found a clear decrease in percentage correct responses as a function of increasing dose of scopolamine $(F(2,27)=11.84, p<0.01)$, and shorter stimulus durations $(F(2,54)=48.42, p<0.01)$. The effect of scopolamine was independent of stimulus duration $(F(4,54)=0.08, n s)$. 

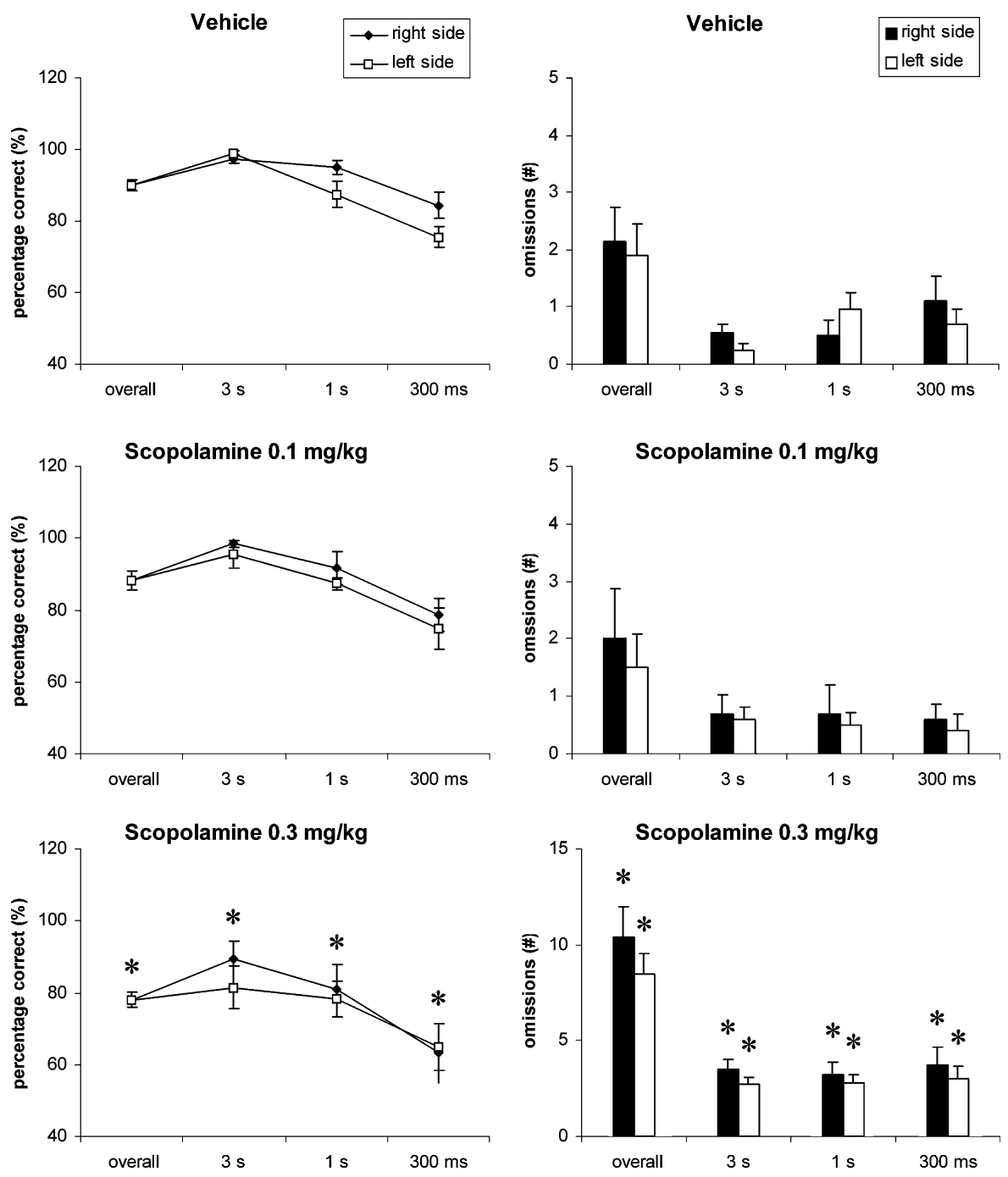

Figure 5. Percentage correct responses and number of omissions subdivided into right-sided and leftsided responses after cholinergic muscarinic receptor blockade with sterile saline (vehicle), scopolamine $0.1 \mathrm{mg} / \mathrm{kg}$ (scopo 0.1), and scopolamine $0.3 \mathrm{mg} / \mathrm{kg}$ (scopo 0.3). Error bars represent the standard error of the mean. Post hoc analyses: $\left({ }^{*}\right)$ significant difference between groups $(p<0.05)$. 
Post hoc analysis revealed that performance at the highest dose (scopolamine 0.3 $\mathrm{mg} / \mathrm{kg}$ ) differed from the other two conditions (vehicle and scopolamine $0.1 \mathrm{mg} / \mathrm{kg}$ ) for all stimulus durations. No differences occurred in responding towards the left or right side. At the shortest stimulus duration, the percentage of correct responses was equal to both sides $(F(1,29)=0.47$, ns $)$. The number of omissions increased with dose of scopolamine $(F(2,29)=27.59, p<0.01)$. Post hoc analysis showed that scopolamine $0.3 \mathrm{mg} / \mathrm{kg}$ differed from both vehicle solution and scopolamine $0.1 \mathrm{mg} / \mathrm{kg}$, whereas no difference was found between vehicle and scopolamine $0.1 \mathrm{mg} / \mathrm{kg}$. No significant effects of Side were found after scopolamine treatment. At the shortest stimulus duration condition, the number of omissions was equal to both sides $(F(1,29)=3.00$, ns).

\section{DISCUSSION}

The aim of the present study was to investigate the effects of right hemisphere cortical infarction on attention performance in the rat. To this end, we trained rats in a behavioural task assessing spatial visual attention, and tested performance after infarction to either the right frontal cortex or right parietal cortex. The main objective was to determine the contribution of a possible attentional impairment to the neglect phenomenon observed previously following cerebral infarction [5]. A secondary objective was to evaluate to the effect of blockade of cholinergic function within our task paradigm.

Our results show a response bias shortly after infarction to frontal and parietal cortices of the right hemisphere (see Figure 3). This effect was transient, since the most pronounced effects were found within the first 4 days after surgery (D4). The percentage of correct responses decreased together with an increased number of omissions, in particular for left-sided trials after right frontal cortex infarction (see Figure 4). The effects of infarction were found only at the shortest stimulus duration, indicating that the observed deficit is related to task difficulty and dependent on attentional load. Furthermore, changes were observed only in the lesion groups and not after sham operation, which shows that performance was not disturbed by the surgical procedures such as anaesthesia. The deficits in attention performance appeared to be a transient phenomenon, since behavioural performance had returned to baseline levels one week after surgery. Deficits in attention performance were also found after muscarinic acetylcholine receptor blockade (Figures 3 and 5). Following systemic administration of scopolamine, response bias also increased together with a decreased percentage of correct responses and an increased number of omissions. 
No differences occurred in responding towards the left or right side. We explain this non-lateralized response bias as a global non-lateralized attentional deficit.

It is tempting to compare the effects of frontal infarction with parietal infarction. First of all it should be noted that direct comparison of potentially dissociable effects of lesion location may be limited by the significant difference in infarct size at the expense of parietal infarction, which was caused by thicker bone of the frontal skull and the attachment of the masseter muscle hindering adequate positioning of the laser beam over the posterior parietal cortex [unpublished observations]. Second, caution should be exercised with making inferences from the figures, as there was no significant difference between lesion groups for index $Y$. Nonetheless, Figure 3 seems to indicate a greater degree of response bias in the parietal lesion group at D4, whereas the decrease in contralateral responses is more pronounced in the frontal lesion group. This apparent paradox is likely due to averaging of individual data since the standard error for index $Y$ is much larger for the parietal lesion group. Another striking feature after parietal infarction is the greater number of omissions compared to frontal infarction, which was equal for both ipsilateral and contralateral omissions. Since a non-lateralized increase in omissions was also observed after scopolamine administration, this may suggest a global attentional deficit related to cholinergic dysfunction following parietal infarction. With regard to hemispatial neglect in humans, parietal infarction in rats may thus induce deficits in non-spatially sustained attention [9, 34]. By contrast, frontal infarction resulted predominantly in a lateralized, contralesional attention deficit. We explain this as an attentional impairment for the left visual field, which is in accordance with the most prevalent form of neglect following right hemisphere damage. It remains speculatory whether this neglect phenomenon involves cholinergic function. Finally, the increase in contralateral correct responses in both lesion groups one week after lesioning (W1) suggests a kind of rebound phenomenon. At this post-lesion time-point, however, neither response bias nor the number of omissions was altered.

Phenomena associated with neglect can be interpreted along the spectrum of sensorimotor integration as either reflecting deficits in attentional performance ('sensory neglect') versus intentional performance ('motor neglect') [35, 36]. In accordance with the latter, we previously reported on a form of motor neglect that lasted for three weeks [5]. We attributed this to impairment of movement initiation that relates well with the functional role of the rat frontal cortex [37]. The present study indicates that the neglect phenomenon after right frontal infarction harbours an attentional component lasting for a few days. A similar short-term effect on attention performance has also been reported following left-hemisphere aspirative lesions of the frontal cortex [38], and may be explained by a transient disturbance in neurotransmitter function.. 
We postulate that the type of visual neglect observed in this study may be caused by a transient disturbance of cholinergic tone within the right hemisphere. As mentioned above, our results do not prove a direct relation between left-sided neglect and cholinergic dysfunction. Prove would require additional experimentation, for instance with direct cortical injection of muscarinic acetylcholine receptor antagonists. All in all, our results clearly show impaired spatial visual attention after cortical infarction as well as after cholinergic blockade.

In conclusion, we compared spatial visual attention performance after right frontal cortex infarction and right parietal cortex in rat brain. Immediately after frontal infarction, a transient impairment in spatial attention of the visual field contralateral to the lesion was observed. We explain this inability as left-sided visual neglect, which may be attributed to impairment of the cortical brain circuitry involved in the processing of attention. Both after parietal infarction and cholinergic blockade with scopolamine, a global attentional deficit occurred. We postulate that dysfunction of the cholinergic system may be related to the observed impairments in spatial visual attention. Our study confirms the role of the parietal and frontal cortices of the rat in attention performance, and corroborates the theory that attention performance is impaired in left-sided visual neglect in human stroke patients. 


\section{REFERENCES}

1. Zilles K, Wree A. Cortex: areal and laminar structure. In: Paxinos G, editor. The Rat Nervous System. San Diego: Academic Press; 1995. p. 649-85.

2. Paxinos G, Watson C. The Rat Brain in Stereotaxic Coordinates. 4th edition ed. New York: Academic Press; 1998.

3. Heilman KM, Valenstein E, Watson RT. Neglect and related disorders. Semin Neurol. 2000;20(4):46370.

4. Swan L. Unilateral spatial neglect. Phys Ther. 2001 Sep;81(9):1572-80.

5. Hoff El, Blokland A, Rutten K, Steinbusch HW, van Oostenbrugge RJ. Dissociable effects in reaction time performance after unilateral cerebral infarction: a comparison between the left and right frontal cortices in rats. Brain Res. 2006 Jan 19;1069(1):182-9.

6. Cherney LR. Unilateral neglect: a disorder of attention. Semin Speech Lang. 2002 May;23(2):117-28.

7. Mesulam MM. A cortical network for directed attention and unilateral neglect. Ann Neurol. 1981 Oct;10(4):309-25.

8. Nachev P, Husain M. Disorders of visual attention and the posterior parietal cortex. Cortex. 2006 Jul;42(5):766-73.

9. Husain M, Rorden C. Non-spatially lateralized mechanisms in hemispatial neglect. Nat Rev Neurosci. 2003 Jan;4(1):26-36.

10. Milner AD, Mclntosh RD. The neurological basis of visual neglect. Curr Opin Neurol. 2005 Dec;18(6):748-53.

11. Corbetta M, Kincade MJ, Lewis C, Snyder AZ, Sapir A. Neural basis and recovery of spatial attention deficits in spatial neglect. Nat Neurosci. 2005 Nov;8(11):1603-10.

12. Mesulam MM. Spatial attention and neglect: parietal, frontal and cingulate contributions to the mental representation and attentional targeting of salient extrapersonal events. Philos Trans R Soc Lond B Biol Sci. 1999 Jul 29;354(1387):1325-46.

13. King VR, Corwin JV. Comparisons of hemi-inattention produced by unilateral lesions of the posterior parietal cortex or medial agranular prefrontal cortex in rats: neglect, extinction, and the role of stimulus distance. Behav Brain Res. 1993 Apr 30;54(2):117-31.

14. Muir JL, Everitt BJ, Robbins TW. The cerebral cortex of the rat and visual attentional function: dissociable effects of mediofrontal, cingulate, anterior dorsolateral, and parietal cortex lesions on a five-choice serial reaction time task. Cereb Cortex. 1996 May-Jun;6(3):470-81.

15. Reep RL, Corwin JV, Cheatwood JL, Van Vleet TM, Heilman KM, Watson RT. A rodent model for investigating the neurobiology of contralateral neglect. Cogn Behav Neurol. 2004 Dec;17(4):191-4.

16. Apicella $P$, Legallet $E$, Nieoullon A, Trouche E. Neglect of contralateral visual stimuli in monkeys with unilateral striatal dopamine depletion. Behav Brain Res. 1991 Dec 20;46(2):187-95.

17. Bushnell PJ, Chiba AA, Oshiro WM. Effects of unilateral removal of basal forebrain cholinergic neurons on cued target detection in rats. Behav Brain Res. 1998 Jan;90(1):57-71.

18. Everitt BJ, Robbins TW. Central cholinergic systems and cognition. Annu Rev Psychol. 1997;48:649-84.

19. Baxter MG, Chiba AA. Cognitive functions of the basal forebrain. Curr Opin Neurobiol. 1999 Apr;9(2):178-83.

20. Sarter M, Givens B, Bruno JP. The cognitive neuroscience of sustained attention: where top-down meets bottom-up. Brain Res Brain Res Rev. 2001 Apr;35(2):146-60.

21. Robbins TW, Everitt BJ, Marston HM, Wilkinson J, Jones GH, Page KJ. Comparative effects of ibotenic acid- and quisqualic acid-induced lesions of the substantia innominata on attentional function in the rat: further implications for the role of the cholinergic neurons of the nucleus basalis in cognitive processes. Behav Brain Res. 1989 Dec 1;35(3):221-40. 
22. Muir JL, Dunnett SB, Robbins TW, Everitt BJ. Attentional functions of the forebrain cholinergic systems: effects of intraventricular hemicholinium, physostigmine, basal forebrain lesions and intracortical grafts on a multiple-choice serial reaction time task. Exp Brain Res. 1992;89(3):611-22.

23. Muir JL, Everitt BJ, Robbins TW. AMPA-induced excitotoxic lesions of the basal forebrain: a significant role for the cortical cholinergic system in attentional function. J Neurosci. 1994 Apr;14(4):2313-26.

24. Muir JL, Everitt BJ, Robbins TW. Reversal of visual attentional dysfunction following lesions of the cholinergic basal forebrain by physostigmine and nicotine but not by the 5-HT3 receptor antagonist, ondansetron. Psychopharmacology (Berl). 1995 Mar;118(1):82-92.

25. Phillips JM, McAlonan K, Robb WG, Brown VJ. Cholinergic neurotransmission influences covert orientation of visuospatial attention in the rat. Psychopharmacology (Berl). 2000 May;150(1):112-6.

26. Gill TM, Sarter M, Givens B. Sustained visual attention performance-associated prefrontal neuronal activity: evidence for cholinergic modulation. J Neurosci. 2000 Jun 15;20(12):4745-57.

27. Himmelheber AM, Sarter M, Bruno JP. Increases in cortical acetylcholine release during sustained attention performance in rats. Brain Res Cogn Brain Res. 2000 Jun;9(3):313-25.

28. Martinez V, Sarter M. Lateralized attentional functions of cortical cholinergic inputs. Behav Neurosci. 2004 Oct;118(5):984-91.

29. Blokland A, Lieben C, Deutz NE. Anxiogenic and depressive-like effects, but no cognitive deficits, after repeated moderate tryptophan depletion in the rat. J Psychopharmacol. 2002 Mar;16(1):39-49.

30. Klapdor K, Blokland A, Horvath E, van der Staay FJ. Bilateral subdural hematoma in the rat: effects on performance in a visual discrimination task. Neurosci Res Comm. 1997;20(2):79-84.

31. Sahgal A. Some limitations of indices derived from signal detection theory: evaluation of an alternative index for measuring bias in memory tasks. Psychopharmacology (Berl). 1987;91(4):517-20.

32. Hoff El, Oude Egbrink MG, Heijnen VV, Steinbusch HW, van Oostenbrugge RJ. In vivo visualization of vascular leakage in photochemically induced cortical infarction. J Neurosci Methods. 2005 Jan 30;141(1):135-41.

33. Vacca LL. Laboratory manual of histochemistry. New York: Raven Press; 1985.

34. Corbetta M, Kincade JM, Shulman GL. Neural systems for visual orienting and their relationships to spatial working memory. J Cogn Neurosci. 2002 Apr 1;14(3):508-23.

35. Kirk A. Spatial neglect. Curr Neurol Neurosci Rep. 2001 Nov;1(6):541-6.

36. Heilman KM. Intentional neglect. Front Biosci. 2004 Jan 1;9:694-705.

37. Kolb B. Functions of the frontal cortex of the rat: a comparative review. Brain Res. 1984 Nov;320(1):6598.

38. Crowne DP, Pathria MN. Some attentional effects of unilateral frontal lesions in the rat. Behav Brain Res. 1982 Sep;6(1):25-39. 


\section{Chapter \\ Pharmacological magnetic resonance imaging of muscarinic acetylcholine receptor activation in rat brain}

Erik I. Hoff

Robert J. van Oostenbrugge Wim M. Otte Ona $\mathrm{Wu}$ Harry W.M. Steinbusch Rick M. Dijkhuizen 


\section{ABSTRACT}

The central cholinergic system is involved in several cognitive functions such as attention, consciousness, learning and memory. Functional imaging of this neurotransmitter system may provide novel opportunities in the diagnosis and evaluation of cognitive disorders. The aim of this study was to investigate the spatial and temporal activation patterns of muscarinic acetylcholine receptor (mAChR) stimulation in rat brain with pharmacological magnetic resonance imaging (phMRI). We performed blood oxygenation level-dependent (BOLD) MRI and contrast-enhanced cerebral blood volume (CBV)-weighted MRI combined with injection of pilocarpine, a nonselective $\mathrm{mAChR}$ agonist, or injection of vehicle solution. BOLD and CBV responses were assessed after pretreatment with methyl-scopolamine in order to block peripheral muscarinic effects. Region-of-interest analysis in individual animals and grouplevel Independent Component Analysis showed that pilocarpine induced significant BOLD signal increases primarily in the cerebral cortex. With contrast-enhanced CBVweighted MRI, positive CBV responses were detected in the cerebral cortex, thalamus, and hippocampus whereas a negative CBV response was observed in the striatum. Thus, pilocarpine induced significant activation responses in brain regions that are known to have a high density of muscarinic receptors. Our study demonstrates that phMRI of mAChR stimulation in rats allows functional assessment of the cholinergic system in vivo. 


\section{INTRODUCTION}

Functional magnetic resonance imaging (fMRI) provides a means to assess brain activity in vivo. Pharmacological MRI (phMRI) is a variant of $\mathrm{FMRI}$ that allows measurement of cerebral activation responses following administration of a brain-targeted drug [1]. PhMRI may be used as a non-invasive assay for neurotransmitter action, as has originally been shown for dopaminergic receptor stimulation [2, 3]. Another neurotransmitter-specific network within the central nervous system, the cholinergic system, modulates several cognitive functions such as attention, motor control, learning and memory. Functional imaging of the cholinergic system could potentially be useful in the diagnostic workup or evaluation of cognitive disorders.

The central cholinergic system consists of a diffuse neuronal network of projections from several basal forebrain nuclei towards the cerebral cortex and hippocampus $[4,5]$. The endogenous neurotransmitter at cholinergic synapses is acetylcholine. Its actions are mediated through nicotinic and muscarinic acetylcholine receptors (mAChRs), which transduce signals via distinct mechanisms. Whereas the functional response to nicotinic receptor stimulation with phMRI has recently been described [68], the activation patterns upon muscarinic receptor stimulation have not been reported previously.

The aim of this study was to investigate the spatial and temporal activation patterns of the cholinergic system in rat brain with phMRI through stimulation of one of its major functional targets: the muscarinic acetylcholine receptor.

\section{MATERIALS AND METHODS}

\section{Animals}

A total of thirty-one adult male Lewis rats were used, weighing 320-380 grams at the time of MRI experiments. All animal surgical and maintenance procedures were approved by the local ethical committee of Utrecht University and met governmental guidelines. All efforts were made to minimize animal suffering and limit the number of animals used.

\section{Experimental design}

Pharmacological MRI involved either blood oxygenation level-dependent (BOLD) MRI or contrast-enhanced cerebral blood volume (CBV)-weighted MRI combined with intravenous administration of the $\mathrm{mAChR}$ agonist pilocarpine. We chose this agent 
because it readily crosses the blood-brain barrier and exerts its cerebral effect almost immediately [9], thereby anticipating a rapid and discriminative MR signal response. Because high dosage of pilocarpine is known to elicit seizure activity [10], we chose to use a low dose of $2.5 \mathrm{mg} / \mathrm{kg}$ that did not evoke epileptic-like behaviour in awake animals nor motion artifacts during MR experiments [data not shown]. Since pilocarpine induces profound peripheral muscarinic effects (increased salivation, emptying of bowel and bladder, and cardiorespiratory changes), all animals, except one group of drug-naïve rats (Group 2 (see below)), were subcutaneously pretreated with methylated scopolamine about 1 hour prior to the MR experiments. Methylscopolamine is a mAChR antagonist that is unable to cross the blood-brain barrier, thereby blocking the peripheral muscarinic effects of pilocarpine. In pilot MRI experiments, we found that intravenous administration of methyl-scopolamine did not evoke significant changes in BOLD and CBV-weighted signal in the brain [data not shown]. Preliminary dose-titration experiments using contrast-enhanced CBV-weighted MRI in methyl-scopolamine pretreated animals demonstrated absence of robust MR signal changes with pilocarpine dosages below $2.5 \mathrm{mg} / \mathrm{kg}$ (resp. $0.025 \mathrm{mg} / \mathrm{kg}, 0.25$ $\mathrm{mg} / \mathrm{kg}$, and $1.25 \mathrm{mg} / \mathrm{kg}$ ) [data not shown].

Experiments were performed in five groups of animals. First, BOLD MRI was conducted in combination with injection of i) vehicle solution of sterile saline in rats pretreated with methyl-scopolamine (Group 1; n=5); ii) pilocarpine in drug-naïve rats (Group 2; $n=5$ ); and iii) pilocarpine in methyl-scopolamine pretreated rats (Group 3; $\mathrm{n}=5$ ). Second, contrast-enhanced CBV-weighted MRI was conducted in combination with injection of i) vehicle (Group 4; $n=5$ ); or ii) pilocarpine (Group 5, n=5) in methylscopolamine pretreated rats.

The arterial blood pressure response to pilocarpine was recorded offline, either with $(n=3)$ or without methyl-scopolamine pretreatment $(n=3)$. The rats were anesthetized with isoflurane, intubated endotracheally and mechanically ventilated with $3 \%$ isoflurane in $\mathrm{O} 2 / \mathrm{N} 2 \mathrm{O}(1 / 2)$. The right femoral artery was cannulated for continuous arterial pressure monitoring. A lateral tail vein was cannulated for intravenous administration of pilocarpine. Upon injection, blood pressure was monitored for up to 60 minutes.

\section{MRI experiments}

Rats were anesthetized with isoflurane and intubated endotracheally. A lateral tail vein was cannulated for the intravenous administration of either vehicle solution or $2.5 \mathrm{mg} / \mathrm{kg}$ pilocarpine hydrochloride (Sigma-Aldrich, Zwijndrecht, The Netherlands); a second tail vein was cannulated in animals of Groups 4 and 5 for injection of contrast 
agent. Approximately 60 minutes prior to phMRI, the animals received a subcutaneous injection of $1 \mathrm{mg} / \mathrm{kg}$ scopolamine methyl-bromide (Sigma-Aldrich, Zwijndrecht, The Netherlands). Animals were placed in a stereotaxic head frame and mechanically ventilated with $3 \%$ isoflurane in $\mathrm{O} 2 / \mathrm{N} 2 \mathrm{O}(1 / 2)$. Body temperature was kept constant at $36.5-37.5{ }^{\circ} \mathrm{C}$ using a feed-back regulated heating pad. Heart rate, blood oxygen saturation and expired $\mathrm{CO} 2$ levels were monitored continuously. Normocapnia was maintained by adjusting the ventilation frequency between 45 and 55 min-1 and the maximum ventilation volume between 3 and $4 \mathrm{ml}$. Retrospectively, no significant correlations were found between end-tidal CO2 and change in BOLD or CBVweighted signal.

T2-weighted MRI was performed at 4.7 T (SISCO/Varian systems, Palo Alto, CA, USA) using a spin-echo multi-slice sequence: repetition time $(T R)=3000 \mathrm{~ms}$; echo time $(\mathrm{TE})=20 \mathrm{~ms}$; data matrix $=128 \times 128$ points; field-of-view (FOV) $=35 \times 35$ $\mathrm{mm} 2 ; 16 \times 1.2-\mathrm{mm}$ slices. Repetitive BOLD MRI was performed using a gradient echo multi-slice sequence: $\mathrm{TR}=500 \mathrm{~ms} ; \mathrm{TE}=23 \mathrm{~ms}$; pulse angle $=41^{\circ}$; data matrix $=64 \times 64$ points; FOV $=35 \times 35 \mathrm{~mm} 2 ; 16 \times 1.2-\mathrm{mm}$ slices; temporal resolution $=32$ s. During BOLD MRI, pilocarpine or vehicle was administered after 10 minutes of baseline measurements (20 scans); data acquisition continued for 30 minutes thereafter (60 scans). Contrast-enhanced CBV-weighted MRI was performed using a gradient-echo multi-slice sequence: $\mathrm{TR}=520 \mathrm{~ms}$; TE $=23 \mathrm{~ms}$; pulse angle $=41^{\circ}$; data matrix $=64 \times 64$ points; FOV $=35 \times 35 \mathrm{~mm} 2 ; 20 \times 1.0-\mathrm{mm}$ slices; temporal resolution $=33 \mathrm{~s}$. Following 10 pre-contrast scans, we intravenously injected ultrasmall superparamagnetic iron-oxide particles (Sinerem ${ }^{\circledR}$, Guerbet, Aulnay-sous-Bois, France; $10 \mathrm{mg} / \mathrm{kg}$ ). Contrast-enhanced CBV-weighted phMRI was started 15 minutes after injection of the contrast agent. After 10 minutes of baseline measurements (20 scans), pilocarpine or vehicle was administered and data acquisition continued for 30 minutes thereafter (60 scans). In each experiment, a total volume of $1 \mathrm{ml}$ was injected intravenously over a 60 -second time period. The administration of pilocarpine or vehicle lasted 30 seconds and was followed by flushing with vehicle solution.

\section{Data processing and statistical analysis}

The phMRI response to pilocarpine was analyzed within several regions-of-interest (ROIs). To this end, BOLD and contrast-enhanced CBV-weighted MRI data of each experiment were coregistered to a reference Lewis rat brain using MNI AutoReg software [11]. Relative changes in CBV were calculated from the contrast-enhanced CBV-weighted MR data as described previously [12]. Based on the anatomical distribution of $\mathrm{mAChR}$ in the rat brain [13], the following ROls were defined: sensorimotor 
cortex (24 voxels), striatum (24 voxels), rostromedial hippocampus (10 voxels) and thalamus ( 20 voxels). These ROIs were delineated bilaterally on the raw BOLD data or relative CBV data of each individual MR experiment. Post-injection signal responses were calculated as a percentage difference relative to mean baseline values within each ROI.

In addition, BOLD and contrast-enhanced CBV-weighted phMRI data were processed and analyzed within the framework of a general linear model (GLM) using SPM2 software package (http://www.fil.ion.ucl.ac.uk/spm). For each experiment, anatomical images were first spatially coregistered to a Lewis rat brain template that was created from twenty coronal T2-weighted anatomical images of a control rat. The transformation parameters were subsequently applied to the phMRI time-series data. To increase robustness for small differences in anatomical positioning and brain shape, data were smoothed using a Gaussian kernel with full-width-at-half-maximum of $1.1 \mathrm{~mm}$ (twice the in-plane resolution).

Group-level Independent Components Analysis (ICA) has been shown to be a useful method in extracting components of interest from fMRI data [14, 15]. The principle advantage of this approach is its applicability to fMRI paradigms for which the MR-signal response is not known a priori. In this study, we used the Group ICA of fMRI Toolbox (GIFT) software package (http://icatb.sourceforge.net). The FastlCA algorithm [16] was used to determine 20 independent components at group level. The following algorithm parameters were used (default): independent components = 20; autofill data reduction $=$ yes; epsilon $=0.0001$; iteration $=1000$; approach $=$ defl; $g=$ pow3. Within the experimental groups with pilocarpine administration, this approach produced one or two components showing specific and significant brain activation patterns. Selection of a particular component was based on the following criteria: regional brain activation (sensitivity), no activation of extracranial areas (specificity), and a smooth signal curve shape. Using these specific components as regressors in SPM2, we performed a first level or model fitting analysis. Regressors were assessed in separate design matrices (one for each regressor). The autoregressive model of order 1 , the $A R(1)$ model, was used to calculate a true temporal correlation and to correct for serial correlations. No high-pass filter was applied due to the long pilocarpine activation time-frame. To make inferences about group data, a second level or random effect analysis was performed in SPM2 (one-sample t-test design, no grand mean scaling, global calculation omitted, statistical significance set at $p<0.05$, family-wise error corrected). 


\section{RESULTS}

\section{Blood pressure}

Figure 1 shows the changes in mean arterial blood pressure (MABP) induced by pilocarpine. After pilocarpine injection into drug-naïve animals, MABP rapidly increased to a maximum of $145 \pm 5 \mathrm{mmHg}$ (mean \pm SD) in about 5 minutes and returned to baseline levels within 20-30 minutes. Blocking of peripheral muscarinic receptors by methyl-scopolamine mitigated the elevation in blood pressure to a maximum of $93 \pm 5 \mathrm{mmHg}$, which is within cerebral autoregulation levels.

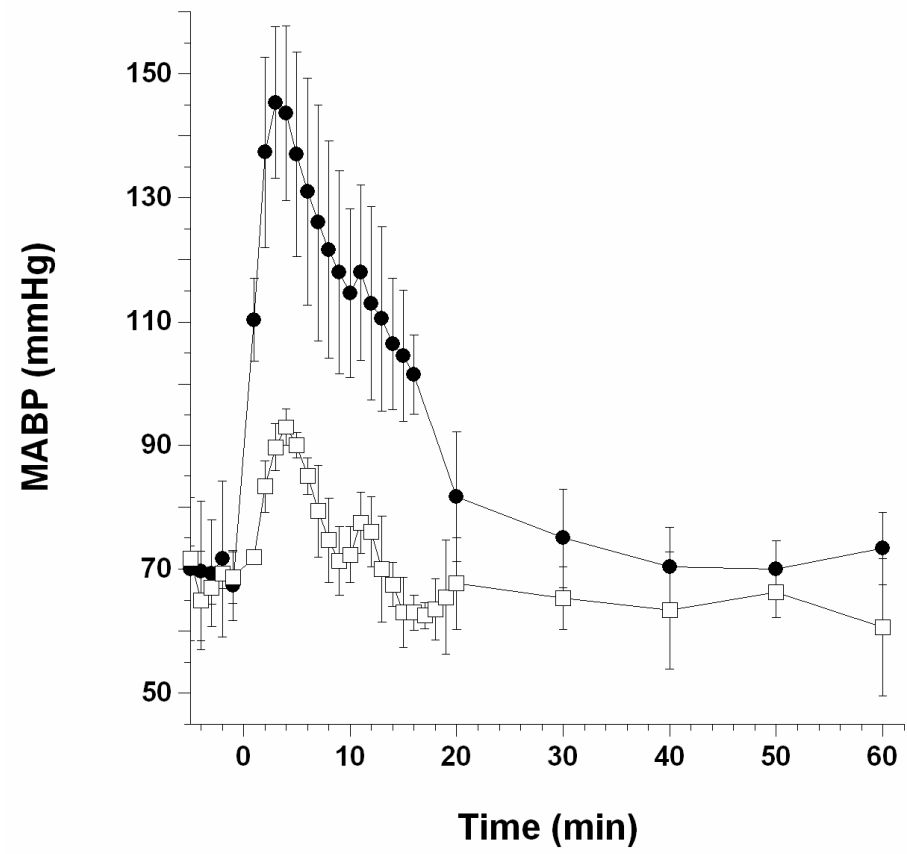

Figure 1. Mean arterial blood pressure responses (MABP, mean $\pm S D$ ) following intravenous administration of pilocarpine in drug-naïve (closed circles) or methyl-scopolamine pretreated Lewis rats (open squares). Pretreatment with methyl-scopolamine mitigated the elevation of MABP to levels within cerebral autoregulation. Note the similarity in baseline MABP between both groups. 


\section{BOLD MRI}

Figure 2 shows the time-courses of changes in BOLD signal in various ROls. A BOLD signal increase upon injection of pilocarpine, starting within the temporal phMRI resolution of 30 seconds, was observed in all ROls, most notably in the cerebral cortex. BOLD signal intensity increased to a maximum in about 1-3 minutes and normalized thereafter. Pilocarpine-induced peak changes in BOLD signal intensity were in the order of $4 \%$ in drug-naïve rats (Group 2) and in the order of $1-2 \%$ in methyl-scopolamine pretreated animals (Group 3). A significant BOLD response was absent following vehicle injection in methylscopolamine pretreated animals (Group 1).

CORTEX

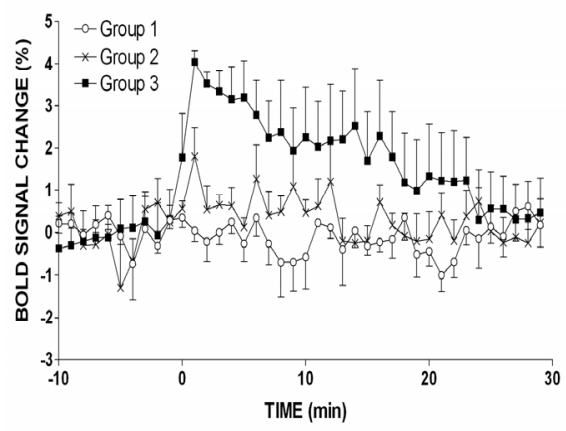

HIPPOCAMPUS

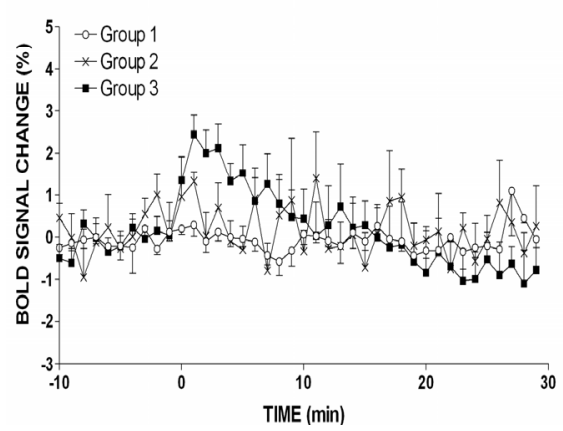

STRIATUM

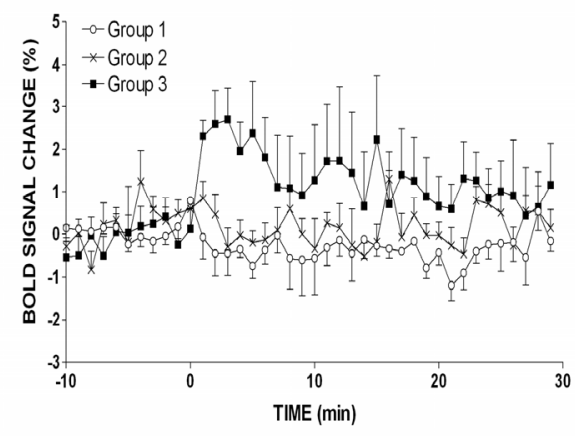

THALAMUS

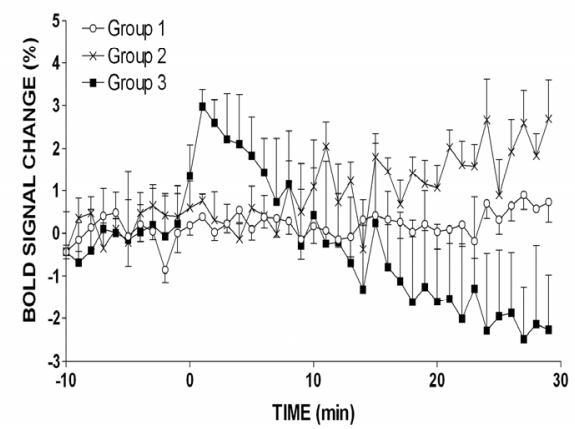

Figure 2. Time-courses of relative BOLD signal intensity changes (mean \pm SEM) in various ROIs upon injection of vehicle in methyl-scopolamine pretreated rats (Group $1(n=5))$ (open circles), and pilocarpine in drug-naïve animals (Group $2(n=5)$ ) (closed squares) and methyl-scopolamine pretreated animals (Group $3(n=5))$ (crosses). 


\section{CBV-weighted MRI}

Prominent pilocarpine-induced activation responses in all ROls were detected with contrast-enhanced CBV-weighted MRI (Group 5), as shown in Figure 3. Positive CBV changes developed rapidly in cortex, hippocampus and thalamus and were strongest in the cortex. Pilocarpine-induced peak CBV changes were in the order of $10-15 \%$. A distinctive negative CBV response was detected in the striatum. In this ROI, CBV dropped by approximately $10 \%$ within $1 \mathrm{~min}$ and slowly recovered thereafter. No significant changes in CBV were observed in response to vehicle injection in control animals (Group 4).
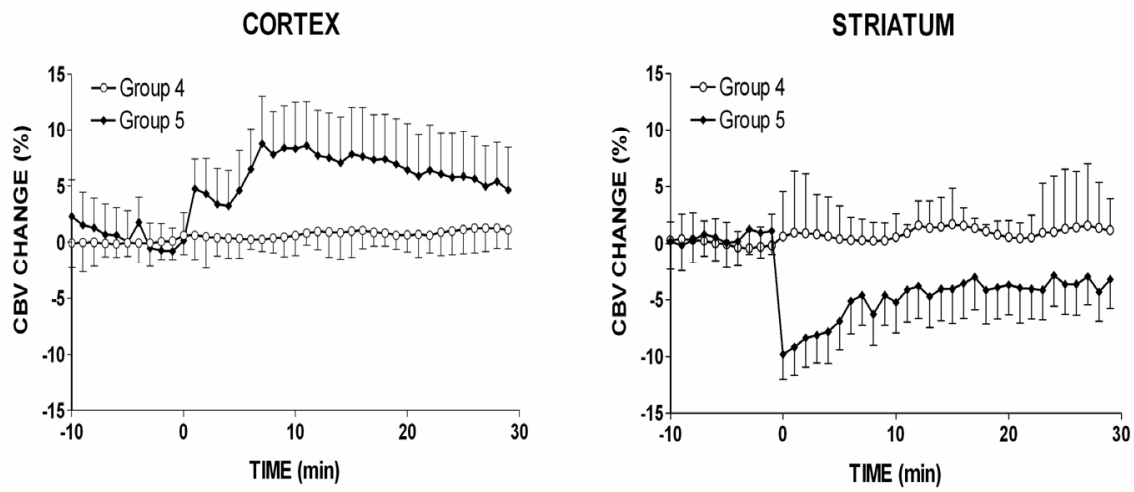

HIPPOCAMPUS

\section{THALAMUS}
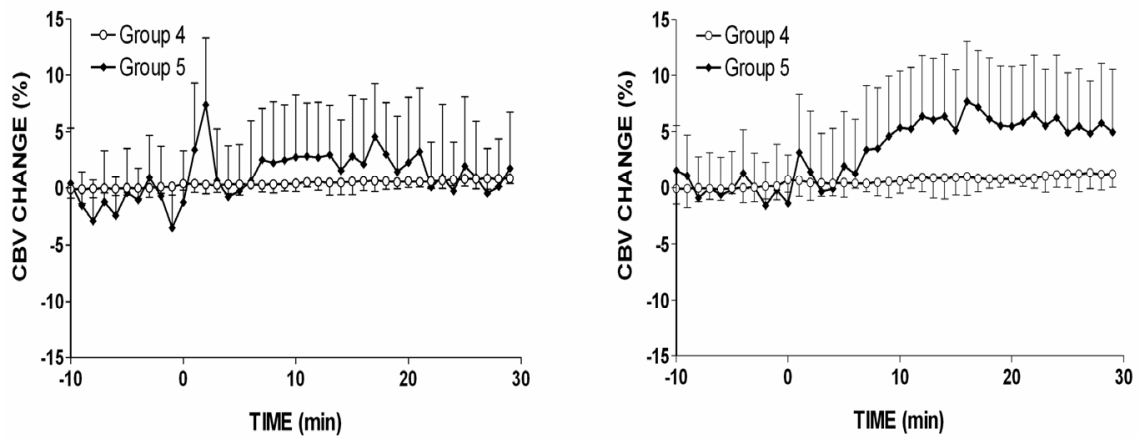

Figure 3. Time-courses of relative changes in CBV for the various ROls (mean \pm SEM) upon injection of vehicle (Group $4(n=5)$ ) (open circles) or pilocarpine (Group $5(n=5)$ ) (closed squares) in methylscopolamine pretreated rats. 


\section{Statistical activation maps}

For the pilocarpine-treated Groups 2 and 3 in the BOLD MRI studies, ICA yielded single components that exhibited a profile that spatially and temporally corresponded to the selection criteria described in the 'Materials and Methods' section (see Figure 4). For the contrast-enhanced CBV-weighted phMRI studies with pilocarpine administration (Group 5), two components showing opposite response profiles were found (see Figure 4).

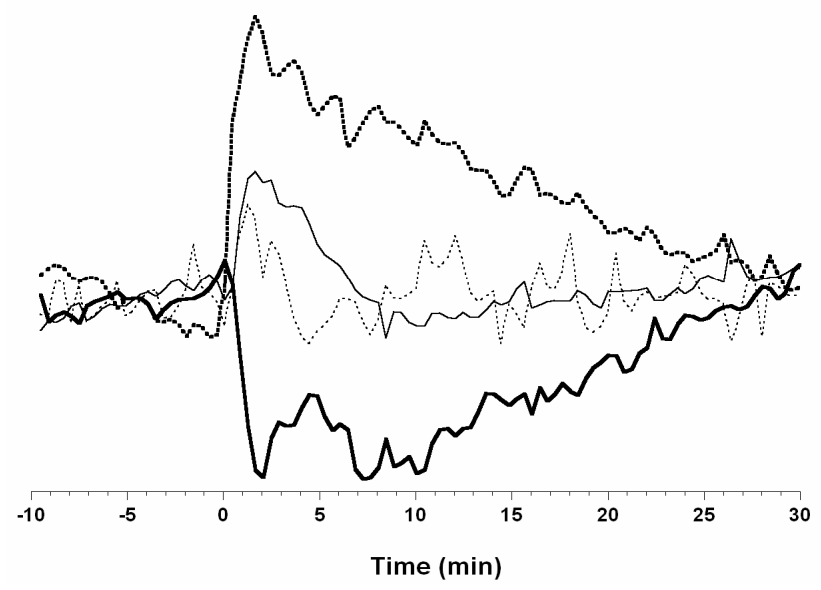

Figure 4. Regressors as determined by group-level independent components analysis (ICA) of phMRI data. Thin lines depict the regressors for pilocarpine-induced BOLD phMRI changes with (Group 3) (dashed line) and without peripheral muscarinic blockade (Group 2) (solid line). Thick solid and dashed lines depict the two regressors for pilocarpine-induced changes with contrast-enhanced CBV-weighted phMRI in methylscopolamine pretreated rats (Group 5).

Figure 5 shows the activation maps that were calculated using the components determined by ICA as regressors in SPM analysis. In drug-naïve (Group 2) and methylscopolamine pretreated rats (Group 3), a significant positive BOLD response upon pilocarpine injection was primarily evident in the cerebral cortex. This was not observed in methyl-scopolamine pretreated control animals that received vehicle solution (Group 1). With contrast-enhanced CBV-weighted MR imaging after pilocarpine injection (Group 5), significant responses in the cortex were associated with an MR signal decrease corresponding to an increase in CBV. In contrast, a significant increase in MR signal (i.e., CBV decrease) was found in the striatum. No significant positive or negative responses were observed in control animals that received vehicle solution (Group 4). 


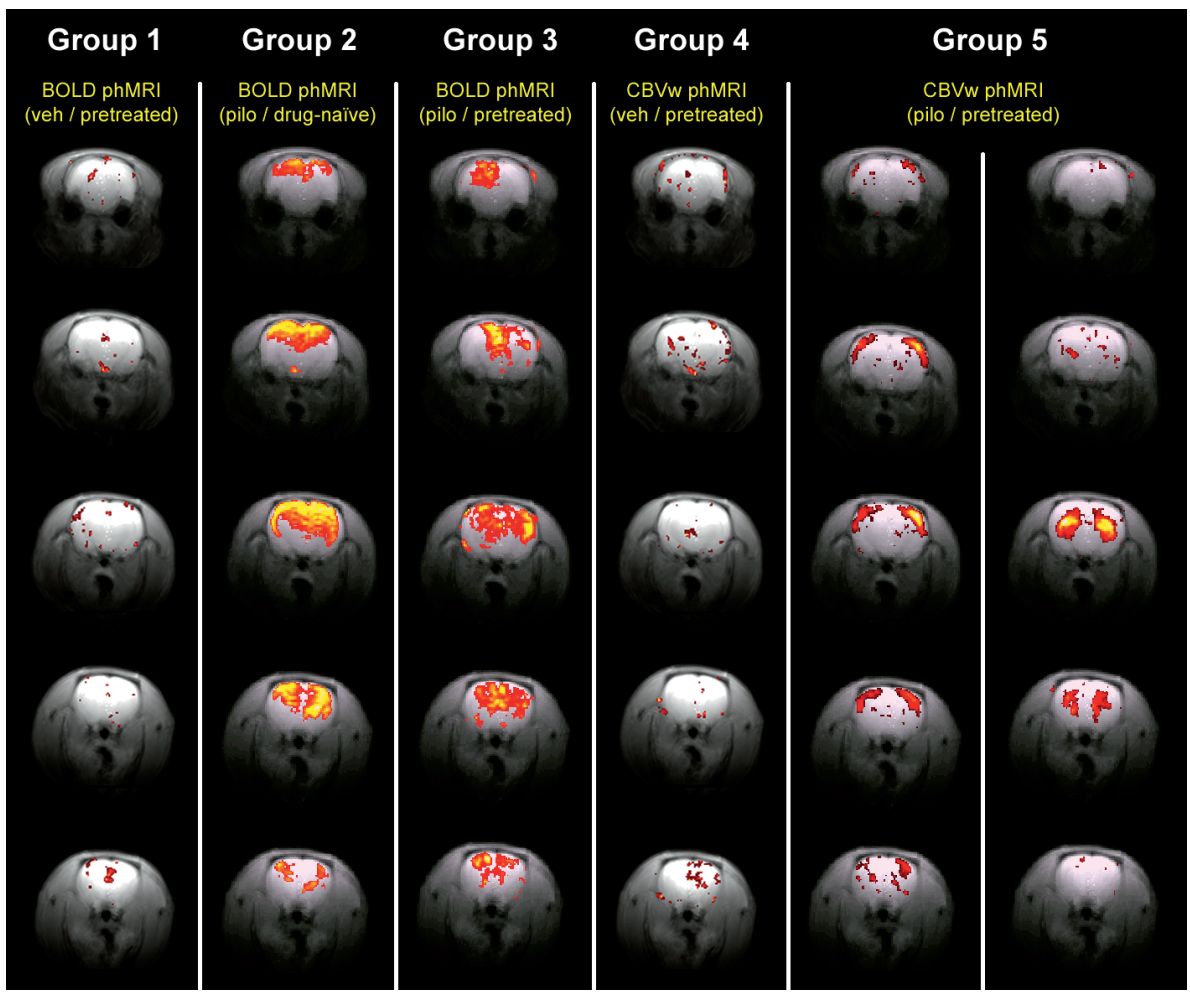

Figure 5. Statistical activation maps of pilocarpine-induced phMRI responses calculated from the regressors shown in Fig 4. First column: BOLD phMRI with vehicle injection (methyl-scopolamine pretreated) (Group 1). Second column: BOLD phMRI with pilocarpine injection (drug-naïve) (Group 2). Third column: BOLD phMRI with pilocarpine injection (methyl-scopolamine pretreated) (Group 3). Fourth column: CBV-weighted phMRI with vehicle injection (methyl-scopolamine pretreated) (Group 4), first regressor. Fifth column: CBV-weighted phMRI with pilocarpine injection (methyl-scopolamine pretreated) (Group 5), first regressor. Sixth column: CBV-weighted phMRI with pilocarpine injection (methyl-scopolamine pretreated) (Group 5), second regressor. Z-values are shown along a color spectrum from red (first to third column: $z=2$; fourth column: $z=-2$ ) to yellow (first to third column: $z=10$; fourth column: $z=-10$ ). 


\section{DISCUSSION}

The present study demonstrates that pharmacological MRI (phMRI) with the muscarinic acetylcholine receptor ( $\mathrm{mAChR}$ ) agonist pilocarpine allows in vivo assessment of $\mathrm{mAChR}$ stimulation in rat brain. Within 30 seconds up to 30 minutes after injection of pilocarpine, regional BOLD and CBV responses were detected in cerebral cortex, striatum, hippocampus and thalamus. Because the pattern of phMRI responses to pilocarpine is undetermined, we applied data-driven independent component analysis (ICA) to map the activation responses in the brain. Group-level ICA of fMRI data consistently yielded one or two components that met our selection criteria and effectively corresponded with plausible pilocarpine-induced activation patterns. By using the GIFT-components as regressors in SPM analysis, statistical maps exhibited significant activation in brain areas with known high $\mathrm{mAChR}$ density: cerebral cortex, striatum, hippocampus and thalamus [13]. The profile of these regressors corresponded with the time-course of phMRI signal changes in our specified ROIs.

The muscarinic class of acetylcholine receptors are widely distributed throughout the body and subserve numerous vital functions in both the brain and autonomic nervous system. Five distinct mAChR subtypes have been identified, designated as M1 through M5 [17]. Both their absolute density and relative proportion decline towards caudal regions of the brain. They are primarily expressed in the cerebral cortex (mostly M1), striatum (mostly M4), hippocampus and thalamus [13, 18]. Pilocarpine is a non-selective mAChR agonist. The significant BOLD and CBV-weighted MR signal responses we observed in the cerebral cortex are consistent with the notion that this brain region holds the highest density of mAChRs. Pilocarpine-induced phMRI responses in the brain may be explained either by blood flow increase upon neuronal activation of $\mathrm{mAChR}$ in the central nervous system or by direct hemodynamic effects caused by stimulation of peripheral $\mathrm{mAChR}$ on cortical microvasculature. Cortical microvessels are endowed with mAChRs [19] and stimulation of those receptors results in microvessel dilation and increased cortical perfusion [20-22].

Our pilocarpine phMRI studies included two different functional MRI methods. First, we assessed the contribution of peripheral mAChR stimulation on phMRIdetected responses in the brain using BOLD MRI. We compared pilocarpine-induced BOLD changes with and without subcutaneous pretreatment with methylscopolamine, a peripheral $\mathrm{mAChR}$ antagonist that is unable to cross the blood-brain barrier, and does not evoke significant cerebral phMRI responses. The observed reduction in pilocarpine-induced phMRI responses following methyl-scopolamine pretreatment, indicates a substantial contribution of peripheral effects on phMRIbased measurement of pilocarpine-induced brain activation. For the subsequent 
study where we compared BOLD MRI versus contrast-enhanced CBV-weighted MRI, we therefore measured pilocarpine-induced activation after peripheral mAChR blockade. Activation responses in cerebral cortex, thalamus and hippocampus were more robustly detected with contrast-enhanced CBV-weighted MRI. Furthermore, a strong decrease in CBV was observed in the striatum; such a negative signal response was not observed with BOLD MRI.

The regionally specific brain signal changes are not likely to be primarily caused by the elevation of blood pressure upon pilocarpine administration. First, blocking of peripheral mAChRs with methyl-scopolamine mitigated the pilocarpine-induced increases in blood pressure to within probable limits of cerebral autoregulation, which has been shown to be preserved in cortex and subcortex at the applied isoflurane anesthesia levels [23]. Second, the sole increase of MABP has been shown to have little effect on BOLD or CBV-weighted MRI signal in rat brain [24].

Contrast-enhanced CBV-weighted phMRI showed robust pilocarpine-induced signal responses in cortical and subcortical regions. This corresponds with studies by Mandeville et al. [25], who have demonstrated enhanced sensitivity with the contrastenhanced CBV-based method compared to BOLD MRI, particularly for activation responses in subcortical structures. Interestingly, in contrast to the positive CBV response in the cerebral cortex, CBV decreased in the striatum following pilocarpine injection. An explanation for the lowering of CBV in the striatum might be the occurrence of a hemodynamic steal as a result of the strong increase in cortical perfusion. An alternative explanation may be related to the distribution of muscarinic receptor subtypes. As mentioned earlier, the M1 receptor subtype predominates in the cerebral cortex whereas the M4 subtype is found primarily in the corpus striatum. Functionally, the odd-numbered mAChR (M1, M3, and M5) are thought to mediate excitatory synaptic transmission whereas the even-numbered receptors (M2 and M4) have been associated with inhibitory synaptic transmission [26]. Specifically, mAChR knock-out experiments in mice have shown that the M1 receptor modulates neurotransmitter signalling in the cortex whilst the M4 receptor acts as an inhibitory autoreceptor in the striatum [27]. Stimulation of M1 may thus produce a cortical response opposite to an M4-mediated striatal response.

In conclusion, our study shows that phMRI with pilocarpine induced significant activation responses in rat brain regions known to have a high density of muscarinic receptors, thereby demonstrating that activation of the cholinergic system can be assessed in vivo. This may provide a potential novel means for functional assessment of the cholinergic system in humans. Ultimately, this approach could aid in the diagnosis of cognitive disorders such as Alzheimers' disease and vascular cognitive impairment. 


\section{REFERENCES}

1. Salmeron BJ, Stein EA. Pharmacological applications of magnetic resonance imaging. Psychopharmacol Bull. 2002 Winter;36(1):102-29.

2. Chen Q, Andersen AS, Zhang Z, Ovadia A, Gash DM, J. AM. Mapping drug-induced changes in cerebral R2* by Multiple Gradient Recalled Echo functional MRI. Magn Reson Imaging. 1996;14(5):469-76.

3. Chen YC, Galpern WR, Brownell AL, Matthews RT, Bogdanov M, Isacson O, et al. Detection of dopaminergic neurotransmitter activity using pharmacologic MRI: correlation with PET, microdialysis, and behavioral data. Magn Reson Med. 1997 Sep;38(3):389-98.

4. Baxter MG, Chiba AA. Cognitive functions of the basal forebrain. Curr Opin Neurobiol. 1999 Apr;9(2):178-83.

5. Everitt BJ, Robbins TW. Central cholinergic systems and cognition. Annu Rev Psychol. 1997;48:649-84.

6. Gozzi A, Schwarz A, Reese T, Bertani S, Crestan V, Bifone A. Region-specific effects of nicotine on brain activity: a pharmacological MRI study in the drug-naive rat. Neuropsychopharmacology. 2006 Aug;31(8):1690-703.

7. Choi JK, Mandeville JB, Chen YI, Kim YR, Jenkins BG. High resolution spatial mapping of nicotine action using pharmacologic magnetic resonance imaging. Synapse. 2006 Aug;60(2):152-7.

8. Skoubis PD, Hradil V, Chin CL, Luo Y, Fox GB, McGaraughty S. Mapping brain activity following administration of a nicotinic acetylcholine receptor agonist, ABT-594, using functional magnetic resonance imaging in awake rats. Neuroscience. 2006;137(2):583-91.

9. Heller Brown J, Taylor P. Muscarinic receptor agonists and antagonists. In: Hardman JG, Limbird LE, Molinoff PB, Ruddon RW, Gilman AG, editors. Goodman and Gilman's: The Pharmacological Basis of Therapeutics. 9th edition ed. New York: McGraw Hill; 1996. p. 155-73.

10. Leite JP, Garcia-Cairasco N, Cavalheiro EA. New insights from the use of pilocarpine and kainate models. Epilepsy Res. 2002 Jun;50(1-2):93-103.

11. Collins DL, Neelin P, Peters TM, Evans AC. Automatic 3D intersubject registration of MR volumetric data in standardized Talairach space. J Comput Assist Tomogr. 1994 Mar-Apr;18(2):192-205.

12. Mandeville JB, Marota JJ, Kosofsky BE, Keltner JR, Weissleder R, Rosen BR, et al. Dynamic functional imaging of relative cerebral blood volume during rat forepaw stimulation. Magn Reson Med. 1998 Apr;39(4):615-24.

13. Tohyama M, Takatsuji K, editors. Atlas of Neuroactive Substances and Their Receptors in the Rat. Oxford: Oxford University Press; 1998.

14. Calhoun VD, Adali T, Pearlson GD, Pekar JJ. A method for making group inferences from functional MRI data using independent component analysis. Hum Brain Mapp. 2001 Nov;14(3):140-51.

15. Calhoun VD, Adali T, Pekar JJ, Pearlson GD. Latency (in)sensitive ICA. Group independent component analysis of fMRI data in the temporal frequency domain. Neuroimage. $2003 \mathrm{Nov} ; 20(3): 1661-9$.

16. Hyvarinen A, Oja E. Independent component analysis: algorithms and applications. Neural Netw. 2000 May-Jun;13(4-5):411-30.

17. Bonner TI, Buckley NJ, Young AC, Brann MR. Identification of a family of muscarinic acetylcholine receptor genes. Science. 1987 Jul 31;237(4814):527-32.

18. Ehlert FJ, Roeske WR, Yamamura HI. Molecular Biology, Pharmacology, and Brain Distribution of Subtypes of the Muscarinic Receptor. In: Bloom FE, Kupfer DJ, editors. Psychopharmacology: The Fourth Generation of Progress. New York: Raven Press; 1995. p. 111-24.

19. Dauphin F, MacKenzie ET. Cholinergic and vasoactive intestinal polypeptidergic innervation of the cerebral arteries. Pharmacol Ther. 1995;67(3):385-417.

20. Hamel E. Cholinergic modulation of the cortical microvascular bed. Prog Brain Res. 2004;145:171-8.

21. Descarries L, Krnjevic K, Steriade M, editors. Acetylcholine in the Cerebral Cortex. Amsterdam: Elsevier B.V.; 2003. 
22. Elhusseiny A, Cohen Z, Olivier A, Stanimirovic DB, Hamel E. Functional acetylcholine muscarinic receptor subtypes in human brain microcirculation: identification and cellular localization. J Cereb Blood Flow Metab. 1999 Jul;19(7):794-802.

23. Lee JG, Hudetz AG, Smith JJ, Hillard CJ, Bosnjak ZJ, Kampine JP. The effects of halothane and isoflurane on cerebrocortical microcirculation and autoregulation as assessed by laser-Doppler flowmetry. Anesth Analg. 1994 Jul;79(1):58-65.

24. Luo F, Wu G, Li Z, Li SJ. Characterization of effects of mean arterial blood pressure induced by cocaine and cocaine methiodide on BOLD signals in rat brain. Magn Reson Med. 2003 Feb;49(2):264-70.

25. Mandeville JB, Jenkins BG, Kosofsky BE, Moskowitz MA, Rosen BR, Marota JJ. Regional sensitivity and coupling of BOLD and CBV changes during stimulation of rat brain. Magn Reson Med. 2001 Mar;45(3):443-7.

26. McKinney M, Coyle JT. The potential for muscarinic receptor subtype-specific pharmacotherapy for Alzheimer's disease. Mayo Clin Proc. 1991 Dec;66(12):1225-37.

27. Bymaster FP, McKinzie DL, Felder CC, Wess J. Use of M1-M5 muscarinic receptor knockout mice as novel tools to delineate the phsyiological roles of the muscarinic cholinergic system. Neurochem Res. 2003;28(3-4):437-42. 



\section{Chapter \\ Alterations in the cholinergic system after frontal cortex infarction in rat brain: pharmacological magnetic resonance imaging of muscarinic receptor activation and stereological analysis of cholinergic basal forebrain neurons}

Erik I. Hoff Harry W.M. Steinbusch Robert J. van Oostenbrugge

Lillian Garrett Wim Otte Kajo van der Marel Rick M. Dijkhuizen 


\begin{abstract}
Vascular cognitive impairment has been related to dysfunction of the central cholinergic system. Studies exploring the putative relationship between vascular cognitive impairment and cholinergic dysfunction have largely been aimed at symptomatic cholinergic treatment rather than focusing on etiological and pathological factors. The present study characterizes subacute responses of the cholinergic system to focal cerebral infarction, investigating changes in muscarinic receptor activation and cholinergic cell number after photothrombotic infarction of the frontal cortex in rat brain. We conducted pharmacological magnetic resonance imaging (phMRI) to assess muscarinic receptor activation at 1 and 3 weeks after photothrombotic infarction in either the left of right frontal cortex. In addition, stereological assessment was performed on choline acetyltransferase (ChAT)-immunostained sections to determine cholinergic cell body count and volume in several basal forebrain nuclei at 4 weeks after infarction. Reductions in muscarinic receptor activation were observed both inside the ischemic area at 1 and 3 weeks, and in areas distant from the lesion at 3 weeks after right-sided frontal cortex infarction. We did not find changes in cholinergic cell number and volume. These results suggest that phMRI is a sensitive tool to detect alterations in cholinergic receptor function, without significant accompanying structural damage to the cholinergic system, following cerebral ischemia. Since the projecting cholinergic cells do not seem to degenerate after focal infarction, functional imaging of muscarinic receptor activation might be of interest for diagnosis and evaluation of therapy in vascular cognitive impairment.
\end{abstract}




\section{INTRODUCTION}

Cognitive impairment related to cerebrovascular disease has received much attention in recent years with terminology such as vascular cognitive impairment or vascular dementia. Vascular cognitive impairment has been linked to cholinergic dysfunction, as cognitive performance is modulated by the brain's cholinergic system [1-3]. Studies exploring the putative relationship between vascular cognitive impairment and cholinergic dysfunction have largely been aimed at symptomatic cholinergic treatment rather than focusing on etiological and pathological factors [4].

The central cholinergic system projects from several basal forebrain nuclei primarily towards the cerebral cortex and hippocampus, both in humans and in lower vertebrates like the rat $[5,6]$. Recently, we have shown that several cognitive impairments seen in human stroke patients can be modelled in rats by measuring reaction time responding and attention performance after cortical infarction [7, 8]. Experimental studies on the degeneration of the basal forebrain cholinergic system in the adult rat have focused on two components of this system: a) the basalo-cortical pathway, from the basal nucleus towards the neocortex, and b) the septo-hippocampal pathway, from the medial septal nucleus towards the hippocampus. The rat cerebral cortex receives its cholinergic input mainly from neurons of the basal nucleus. The cholinergic basalo-cortical pathway does not follow a well-defined route like the septo-hippocampal pathway, but radiates out medially and laterally from the basal forebrain. Damage to these two pathways causes anterograde and retrograde axonal degeneration within the first four weeks [Henderson, 1996], which may result in cell death of cholinergic neurons. It has been reported that secondary cell death does not occur in the basal nucleus after cortical lesioning, supposedly because the cells of the basalo-cortical pathway have more widespread projections [9].

The endogenous neurotransmitter at cholinergic synapses is acetylcholine, which actions are mediated through nicotinic and muscarinic acetylcholine receptors. Previously, we have demonstrated the feasibility of pharmacological functional magnetic resonance imaging (phMRI) for functional assessment of muscarinic receptor activation in rats in vivo. PhMRI is a variant of functional MRI that allows measurement of cerebral activation responses following administration of a brain-targeted drug [10]. Immunohistochemistry and autoradiography studies have reported reduced numbers of muscarinic receptors and muscarinic receptor binding in the ipsilateral hemisphere after ischemia to the frontoparietal cortex, presumably through the degeneration of the basalo-cortical pathway [11-15].

In the present study, we investigated structural and functional alterations of the cholinergic system after focal infarction of the frontal cortex in rats. We hypothesized that 
cortical ischemia would result in reduced cortical muscarinic receptor activation and a reduced number of cortically projecting cholinergic cells. Therefore we characterized subacute changes in the cholinergic system after cortical infarction by measurement of muscarinic receptor activation and cholinergic cell number both in- and outside the infarcted area.

\section{MATERIALS AND METHODS}

\section{Animals}

Animal surgical and maintenance procedures were approved by the local ethical committees of Maastricht University (Maastricht, The Netherlands) and Utrecht University (Utrecht, The Netherlands) and met Dutch governmental guidelines. All experiments were conducted with adult male Lewis rats weighing 280-320 g at the start of the experiments. We performed two types of experiments. In one experiment, phMRI of cerebral muscarinic receptor activation was conducted in sham-operated rats and in animals after unilateral photothrombotic infarction to either the right or left frontal cortex (Experiment I). In another experiment, we determined cholinergic cell number in several basal forebrain nuclei following sham-operation or after unilateral photothrombotic infarction to the right or left frontal cortex (Experiment II).

\section{Photothrombotic cortical infarction}

Body weight was matched between experimental groups prior to the induction of photothrombotic cortical infarction or sham operation. Anaesthesia was induced by inhalation of isoflurane $(4 \%)$ and maintained with isoflurane $(1 \%)$ in a mixture of O2/N2O (1:2) in spontaneously breathing rats. As described previously [16], cortical infarction was induced by photochemically initiated thrombosis through intravenous injection of the light-sensitive dye Erythrosin B (Sigma-Aldrich, Zwijndrecht, The Netherlands) and illumination of the skull with a monochromatic light source (DPSS laser, Uniphase, Germany). The light source, with a diameter of $2.4 \mathrm{~mm}$, was positioned perpendicular to the skull surface in the centre of a perforated filtration paper that absorbed any leakage of blood. Its beam was positioned at $2.7 \mathrm{~mm}$ anterior and $2.7 \mathrm{~mm}$ lateral to bregma's point for infarction of the frontal cortex. In sham-operated animals, the order of dye injection and illumination was reversed. 


\section{Experiment I-phMRI of muscarinic receptor activation}

Assessment of muscarinic receptor activation using phMRI was performed in five groups of animals: sham-operated $(n=5)$, at 1 and 3 weeks after left frontal cortex infarction ( $n=5$ for each time-point), and at 1 and 3 weeks after right frontal cortex infarction ( $n=5$ for each time-point). Since the administration of the pharmacological agent and the contrast agent are possible confounding factors in repetitive MRI experiments, phMRI was performed as a single-event and not repeated over time in the same animal.

phMRI

Pharmacological MRI combined contrast-enhanced cerebral blood volume (CBV)weighted MRI with intravenous administration of the muscarinic acetylcholine receptor agonist pilocarpine (see chapter 5 ). In short, rats were anesthetized with isoflurane and intubated endotracheally. A lateral tail vein was cannulated for injection of contrast agent. A second tail vein was cannulated for the administration of $2.5 \mathrm{mg} / \mathrm{kg}$ pilocarpine hydrochloride (Sigma-Aldrich, Zwijndrecht, The Netherlands). Animals were placed in a stereotaxic head frame and mechanically ventilated with $3 \%$ isoflurane in $\mathrm{O} 2 / \mathrm{N} 2 \mathrm{O}(1 / 2)$. Body temperature was kept at $36.5-37.5^{\circ} \mathrm{C}$ using a feedback regulated heating pad. Heart rate, blood oxygen saturation and expired $\mathrm{CO} 2$ levels were monitored continuously. Approximately 60 minutes prior to phMRI, we subcutaneously injected $1 \mathrm{mg} / \mathrm{kg}$ scopolamine methyl-bromide (Sigma-Aldrich, Zwijndrecht, The Netherlands), to block peripheral muscarinic effects of pilocarpine (see Chapter 5).

T2-weighted MRI was performed at 4.7 T (SISCO/Varian systems, Palo Alto, CA, USA) using a spin-echo multi-slice sequence: repetition time $(T R)=3000 \mathrm{~ms}$; echo time $(\mathrm{TE})=20 \mathrm{~ms}$; data matrix $=128 \times 128$ points; field-of-view $(\mathrm{FOV})=35 \times 35$ $\mathrm{mm} 2 ; 16 \times 1.2-\mathrm{mm}$ slices. Contrast-enhanced CBV-weighted MRI was performed using a gradient-echo multi-slice sequence: $\mathrm{TR}=520 \mathrm{~ms}$; $\mathrm{TE}=23 \mathrm{~ms}$; pulse angle = $41^{\circ}$; data matrix $=64 \times 64$ points; FOV $=35 \times 35 \mathrm{~mm} 2 ; 20 \times 1.0-\mathrm{mm}$ slices; temporal resolution $=33 \mathrm{~s}$. Following 10 pre-contrast scans, we intravenously injected ultrasmall super paramagnetic iron-oxide particles (Sinerem ${ }^{\circledR}$, Guerbet, Aulnay-sousBois, France; $10 \mathrm{mg} / \mathrm{kg}$ ). The blood half-life of Sinerem ${ }^{\circledR}$ in Lewis rats at this dose is about 5-6 hours [17]. Contrast-enhanced CBV-weighted phMRI was started 15 minutes after injection of contrast agent. Pilocarpine was administered after 10 minutes of baseline measurements (20 scans), and data acquisition continued for 30 minutes thereafter (60 scans). 


\section{Data processing and statistical analysis}

The phMRI response to pilocarpine was analyzed within several regions-of-interest (ROIs). To this end, contrast-enhanced CBV-weighted MRI data of each experiment were coregistered to a rat brain MRI template. Relative changes in CBV were calculated from the contrast-enhanced CBV-weighted MRI data as described previously [18]. Using Paxinos and Watson's rat brain atlas in stereotaxic coordinates [19], the following ROls were delineated bilaterally on the relative CBV maps of each individual: motor cortex (M2), sensory cortex (S2), and striatum (CPu) [see Figure 1].
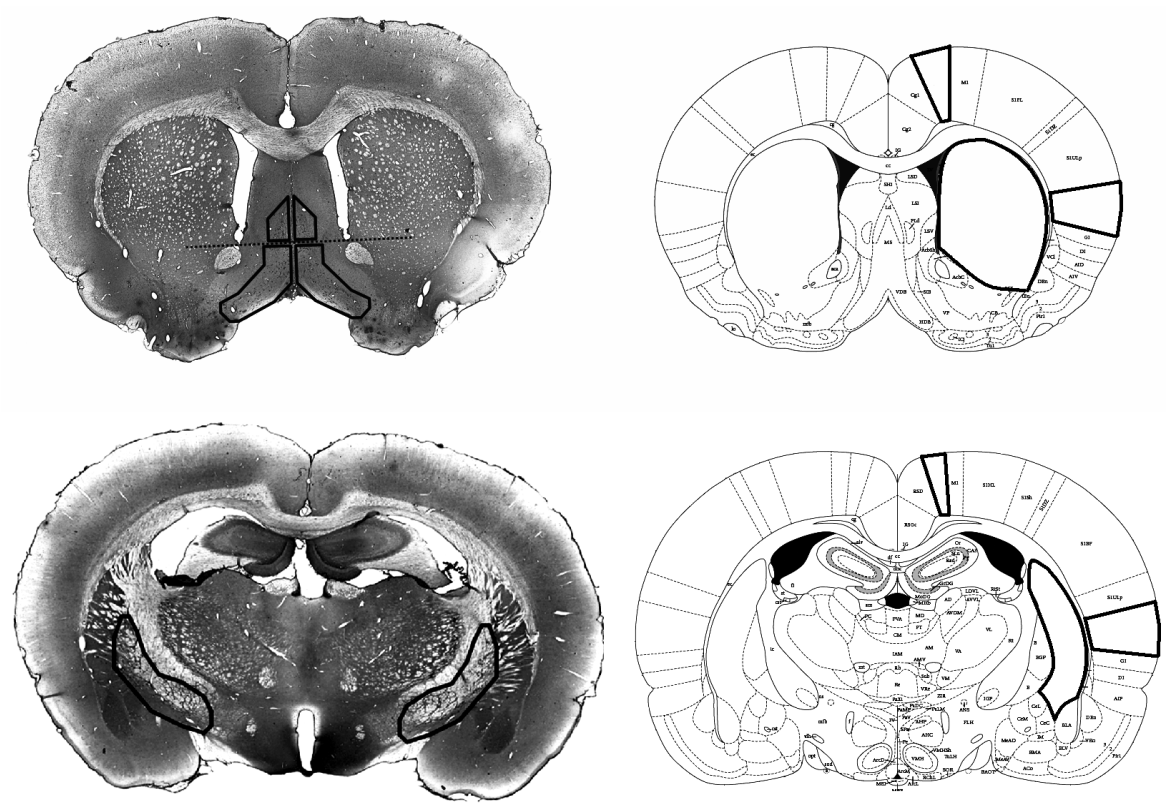

Figure 1. Coronal rat brain sections showing ROls for the analysis of number of ChAT-immunopositive neurons (left images) and muscarinic receptor activation (right images). Upper left image shows medial septum (Ch1) and horizontal/vertical limb of diagonal band of Broca (Ch2/3); note the artificial line connecting the upper parts of anterior commissure that was used to make a reliably distinction between these two ROIs. Lower left image shows the basal nucleus ROI (Ch4). Right images show ROls in motor cortex (M2) and sensory cortex (S2) and striatum (CPu). 
Since the photothrombotic lesions were located in the rostral frontal cortex, the ROI of the motor cortices were subdivided into areas located inside versus outside of the ischemic lesion (3.24 to $2.28 \mathrm{~mm}$ versus 1.08 to $-1.08 \mathrm{~mm}$ anterior/posterior to bregma, respectively). The sensory cortex was delineated from 1.08 to $-1.08 \mathrm{~mm}$ anterior/posterior to bregma, and the striatum from 2.04 to $1.08 \mathrm{~mm}$ anterior/posterior to bregma. Signal responses within each ROI were calculated separately for the left and right hemisphere, as well as combined for both hemispheres. All statistical analyses were done using SPSS software (version 12). The CBV changes over time were analysed in a repeated measures within-subjects ANOVA design using general linear modelling (GLM). Within-subject factor was phMRI time-point after pilocarpine injection (Time - 120 levels) and between-subjects factor was experimental group (Group - 5 levels), followed by a post-hoc LSD correction for multiple comparisons. Data output was split into results by $\mathrm{ROI}$ and results by hemisphere (both, left or right).

Additionally, contrast-enhanced CBV-weighted phMRI data were processed and analyzed within the framework of FMRIB's Software Library (FSL, www.fmrib.ox.ac.uk/fsl). The contrast-enhanced CBV-weighted phMRI time-series were motion corrected with the FMRIB's Linear Image Registration Tool (MCFLIRT; $[20,21])$. To increase robustness for small differences in anatomical positioning and brain shape, data were spatially smoothed using a Gaussian kernel with full-width-athalf-maximum of $1.0 \mathrm{~mm}$ (twice the in-plane resolution) followed by grand-mean intensity normalization of the 4D datasets by a single multiplicative factor. Two main explanatory variables from the sham group were selected from a group-level data analysis using probabilistic independent component analysis as implemented in MELODIC (Multivariate Exploratory Linear Decomposition into Independent Components), part of FSL. The melodic input data were pre-processed as followed: masking of non-brain voxels; voxel-wise de-meaning and normalization of the voxel-wise variance. Pre-processed data were whitened and projected into a multi-dimensional subspace using probabilistic principal component analysis where the number of dimensions was estimated using the Laplace approximation to the Bayesian evidence of the model order [22]. The whitened observations were decomposed into sets of vectors which describe signal variation across the temporal domain (time-courses), the subject domain and the spatial domain (maps) by optimizing for non-Gaussian spatial source distributions using a fixed-point iteration technique [23]. Estimated component maps were divided by the standard deviation of the residual noise and thresholded by fitting a mixture model to the histogram of intensity values [22]. The two selected explanatory variables (for positive and negative pilocarpine-induced CBV-responses, respectively) had explained variance of $11.73 \%$ and $5.37 \%$ respec- 
tively and were smoothed with a 3-th order b-spline function in the Curve Fitting Toolbox (version 1.1.7; Matlab, The MathWorks) prior to inclusion in the design matrix. The model-based phMRI analysis was carried out using FMRI Expert Analysis Tool version 5.91, part of FSL. The analysis contained local autocorrelation correction and first-level GLM time-series analysis. First-level contrast images were subsequently used for high-level analysis with one-sample t-test setup to measure the mean group effect (i.e., to establish whether the group activates on average). For higher-level activation estimation we applied FMRIB's Local Analysis of Mixed Effects (FLAME). FLAME uses Bayesian modelling and estimation. The Z (Gaussianized $T / F$ ) statistic image was thresholded using clusters determined by $Z>2.3$ and a (corrected) cluster significance threshold of $p=0.05$ [24]; Worsley 2001). Finally, $Z$ statistic parametric group maps were overlaid on the structural MRI template used for coregistration.

\section{Experiment II - Immunohistochemistry of cholinergic cells}

Fifteen animals were randomly selected from the experimental groups described in a previous behavioural study [7]: sham-operated animals $(n=5)$, left frontal cortex infarction $(n=5)$, and right frontal cortex infarction $(n=5)$. Age and body weight did not differ between groups. Lesion volume histology and choline acetyltransferase (ChAT) immunohistochemistry were performed at 4 weeks after infarction or sham-operation.

\section{Tissue preparation}

Rats were anaesthetized with sodium pentobarbital (Nembutal®, $60 \mathrm{mg} / \mathrm{kg}$ i.p.). Subsequently, perfusion fixation was performed by transcardial administration of ice-cold $0.1 \mathrm{M}$ tyrode buffer ( $\mathrm{pH} 7.6)$ followed by Somogyi's fixative $(\mathrm{pH} 7.4)$ consisting of $4 \%$ paraformaldehyde, $15 \%$ picric acid and $0.05 \%$ glutardyaldehyde in $0.1 \mathrm{M}$ phosphate buffer at $70 \mathrm{mmHg}$. Brains were removed from the cranium, and immersion-fixed for another 2 hours in Somogyi's fixative. Overnight, brain tissue was cryoprotected by immersion in $15 \%$ sucrose / $0.1 \mathrm{M}$ phosphate buffer at $4{ }^{\circ} \mathrm{C}$. Afterwards, the brains were quickly frozen on $\mathrm{CO} 2$ gas and stored at $-80{ }^{\circ} \mathrm{C}$ until further processing. Brains were cut on a cryostate (Leica CM 3050, Nussloch, Germany) into $30 \mu \mathrm{m}$ coronal sections collected serially. For the quantification of lesion volume every fifth section was stained on slides with a May-Grunwald-Giemsa protocol [25], and calculated as described previously [7]. 


\section{ChAT immunohistochemistry}

For the quantification of cholinergic cells every fourth $30 \mu \mathrm{m}$ free-floating coronal section was stained immunohistochemically for ChAT. In brief, the sections were rinsed three times in Tris-buffered saline (TBS, $0.01 \mathrm{M}$ ) with $0.2 \%$ Triton X-100 (TBST) followed by incubation for 72 hours at $4{ }^{\circ} \mathrm{C}$ with the primary antibody (rat antiChAT, Eckenstein, 1:800) in TBS-T and 3\% donkey serum. After another three rinses in TBS-T, the sections were incubated overnight at $4^{\circ} \mathrm{C}$ with a secondary antibody (biotinylated donkey anti-rat, 1:400) in TBS-T and 3\% donkey serum. Staining was visualized at room temperature for 10 minutes using the $A B C$ method with a vectastain kit (Vector Laboratories, Burlingame, CA), 0.3\% 3'3'-diaminobenzidine (DAB, Sigma), $0.3 \%$ nickelchloride and $0.3 \%$ hydrogenperoxide in TBS-T. Counterstaining was carried out with cresyl violet. Sections were washed with TBS, mounted onto gelatinized glass slides, dehydrated, cleared, and coverslipped using DePeX.

\section{Cell counting and volume estimation}

ChAT-immunopositive neurons were quantified using a stereology work station consisting of a modified light microscope (Olympus BX50; Olympus, Tokyo, Japan), Olympus UPlanApo objectives (10x, NA = 0.40; 100x, oil, NA = 1.35), a motorized specimen stage for automatic sampling (Maerzhauser, Wetzlar, Germany), an electronic microcator (Heidenhain, Traunreut, Germany), a CCD color video camera (JAI, Glostrup, Denmark), a PC with framegrabber board (Screen Machine II, Fast Multimedia, Munich, Germany), stereology software (C.A.S.T.-Grid, Olympus; Stereolnvestigator, MicroBrightField, Inc., Colchester, VT) and a 17-inch television screen monitor.

All ChAT-immunopositive cell bodies were counted in the following ROls according to Mesulam [26]: medial septum (Ch1), horizontal and vertical limbs of the diagonal band of Broca (Ch2 and Ch3), and basal nucleus and substantia innominata (Ch4) [see Figure 1]. These ROI were delineated in both cerebral hemispheres at $\times 10$ magnification on whole-brain sections using The Atlas of the Rat Nervous system [19]. For reliable measurements and adequate number of ChAT-positive cells per $\mathrm{ROI}$, we delineated areas $\mathrm{Ch} 1$ and $\mathrm{Ch} 2 / 3$ between $1.20 \mathrm{~mm}$ (anterior boundary) and $0.24 \mathrm{~mm}$ (posterior boundary) from bregma. When the distinction between these two areas could not be made reliably, we used an artificial division by drawing a straight line connecting the upper parts of anterior commissure. Area Ch4 was delineated between -1.56 $\mathrm{mm}$ (anterior boundary) and $-2.64 \mathrm{~mm}$ (posterior boundary) from bregma, as the alignment of cholinergic cells of the basal nucleus between the exter- 
nal globus pallidus, internal capsule and optic tract. The remainder of cholinergic cells within the horizontal limb of the diagonal band of Broca, the substantia innominata and the basal nucleus were excluded because of either difficulties in reliable delineation or insufficient number of ChAT-positive cell bodies per tissue section. To correct for possible differences in ROI size between hemispheres, the total area of each ROI was calculated for every animal.

Total numbers of ChAT-positive neurons were evaluated with the fractionator at 100x magnification. All neurons which nucleus or soma with axon hillock came into focus within the unbiased virtual counting frames distributed systematically over the entire delineated ROI were counted. Eight serial sections of each rat brain were used to calculate the total number of cholinergic cells within each ROI. Countings were performed in duplicate by two persons blinded for the experimental group. In addition, the optical fractionator was used in a subset of animals to estimate cell body volume ( $n=2$ per group).

Analyses were performed using SPSS (v.12). For each experimental group, mean and standard errors of the mean were calculated for the total number of ChATpositive neurons and their estimated volume. Comparisons between groups were performed with a Student's two-tailed t-test. Statistical significance was set at $p<0.05$.

\section{Statistical analysis}

Analyses were performed using SPSS (version 12). For each experimental group, mean and standard errors of the mean were calculated for the total number of ChATpositive neurons and their estimated volume. Comparisons between groups were performed with a Student's two-tailed t-test. Statistical significance was set at $p<0.05$.

\section{Results}

\section{Experiment I-phMRI of muscarinic receptor activation}

Figure 2 shows T2-weighted MR images of rat brain at 1 week and at 3 weeks after photothrombotic infarction in the frontal cortex. The lesion is characterized as a hyperintense region. One of the 25 animals died during the induction of photothrombotic infarction. Final group number for statistical analysis was: sham-operated animals $(n=5)$, left frontal cortex infarction at 1 week $(n=4)$, left frontal cortex infarction at 3 weeks $(n=5)$, and right frontal cortex infarction at 1 and 3 weeks $(n=5$ in each group). All of the statistical analyses described compare ROI data of the ipsilesional hemisphere after photothrombotic infarction versus after sham operation. 


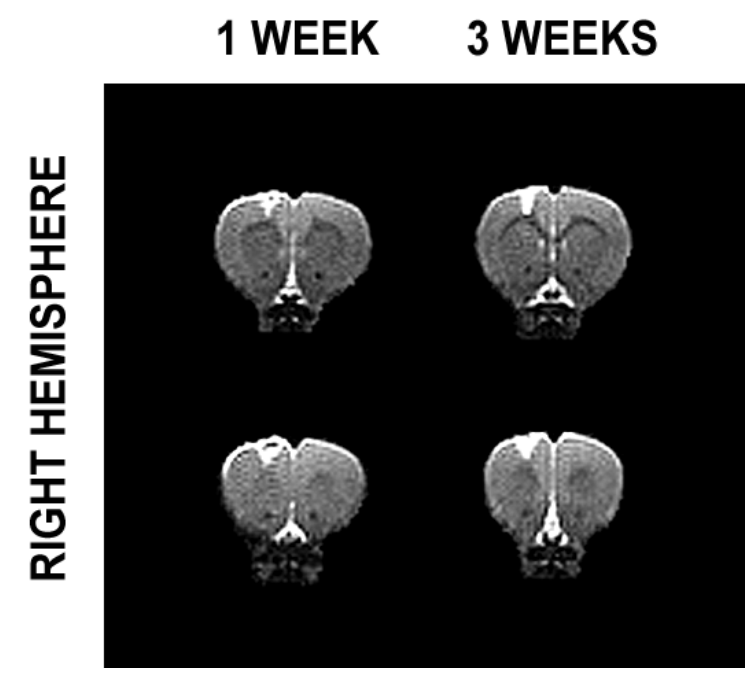

Figure 2. T2-weighted MR images showing right-sided infarction of the cortex in rat brain after 1 week (left) and 3 weeks (right) of photothrombotic infarction.

Figure 3 depicts relative CBV time-courses in different groups and ROls in response to muscarinic receptor stimulation with pilocarpine. Figure 3, first row, shows a reduction in CBV response inside the infarcted area of the motor cortex after photothrombotic stroke to the frontal cortex (Time: $F(79,1501)=1.55, p<0.01$; Time*Group: $F(316,1501)=1.85, p<0.001)$. Post-hoc analysis did not show this to be significant for right-sided lesioned animals at 1 week and 3 weeks $(p=0.065$ and $p=0.055$, respectively) as well as for left-sided lesioned animals ( $p=0.269$ and $p=0.169$, respectively).

Figure 3, second row, shows the time-courses of pilocarpine-induced CBV changes in the sensory cortex distant from photothrombotic infarction to either the left or right frontal cortex (Time: $F(79,1501)=17.26, p<0.001$; Time*Group: $F(316,1501)=3.0, p<0.001)$. CBV changes were not significantly reduced at 1 week post-infarction in the left-hemisphere lesion group $(p=0.130)$ whereas in the righthemisphere lesion group CBV changes were notably reduced at 3 weeks $(p<0.05)$.

Figure 3, third row, shows the time-courses of pilocarpine-induced CBV changes in the ipsilesional striatum after photothrombotic infarction to either the left or right frontal cortex (Time: $F(79,1501)=6.71, p<0.001$; Time*Group: $F(316,1501)=3.01$, $p<0.001)$. Compared to sham-operated animals, a significantly stronger negative CBV response was found in the right-hemisphere lesion group at 3 weeks postinfarction $(p<0.01)$. 

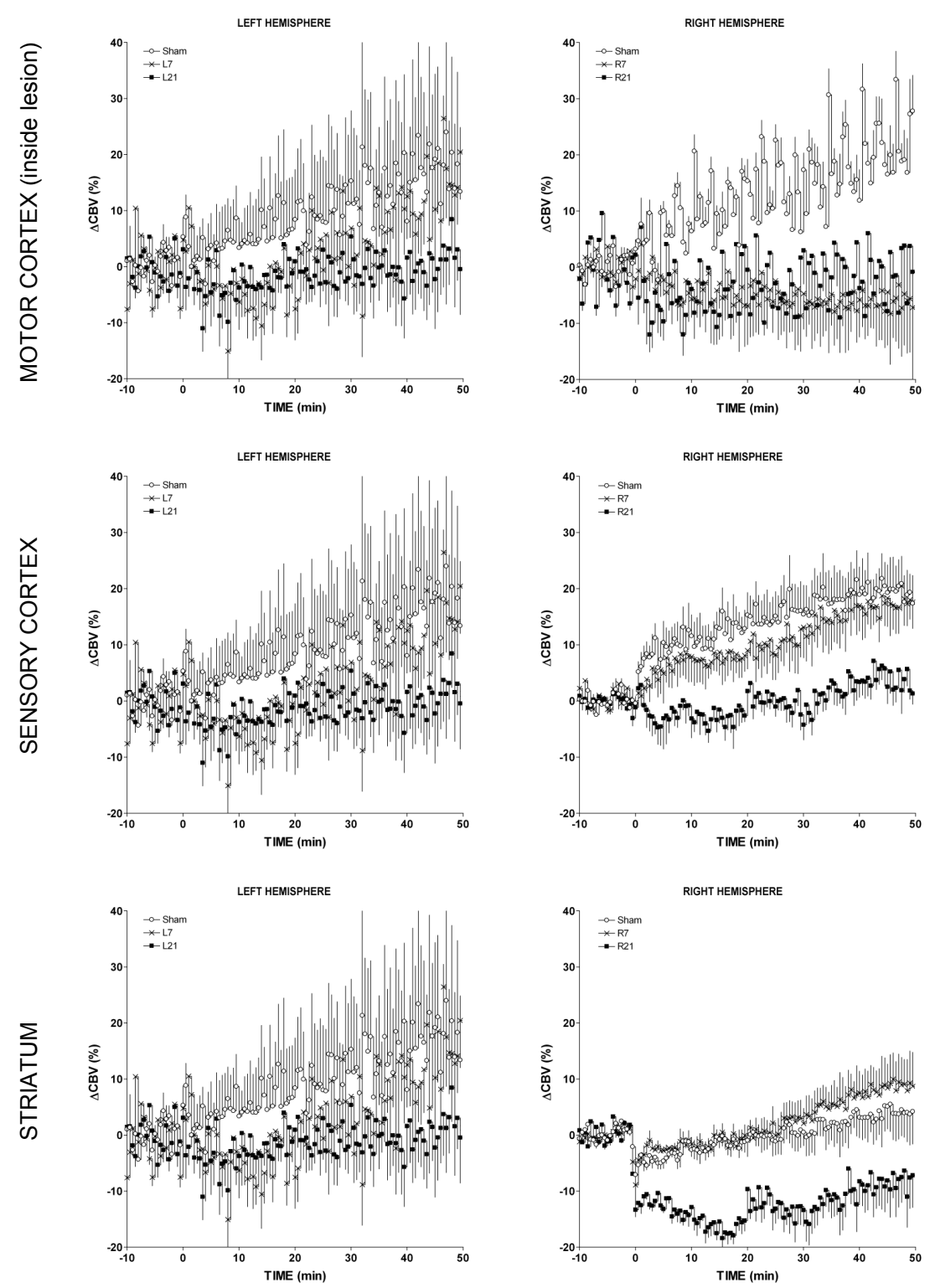

Figure 3. Time-courses of muscarinic receptor stimulation induced CBV changes using pilocarpine. Graphs show CBV responses in various ROls after sham operation (sham), and at 1 week and 3 weeks after left-sided cortical infarction (left column: L7 and L21, respectively) and right-sided cortical infarction (right column: R7 and R21, respectively). ROI data were extracted from the ipsilesional hemisphere and compared to the ipsilateral side in sham-operated animals. Error bars represent standard error of the mean. 


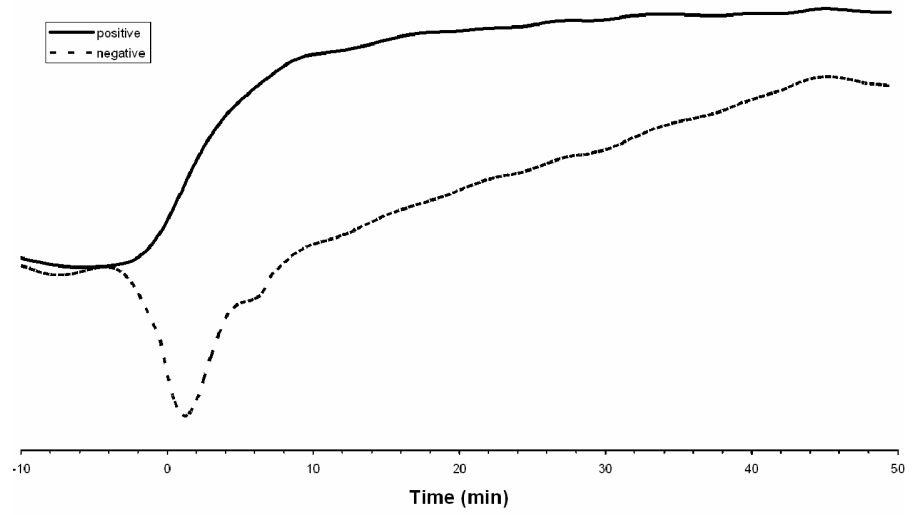

Figure 4. Regressors derived from FSL analyses: positive and negative signal components

Figure 4 depicts the regressors as derived from pharmacological MRI data analysis in FSL. Figure 5 shows the activation maps that were calculated using these regressors in the control group of sham-operated animals. Positive CBV responses were noted in the posterolateral cerebral cortex whereas negative activation responses were found mainly in the striatum.
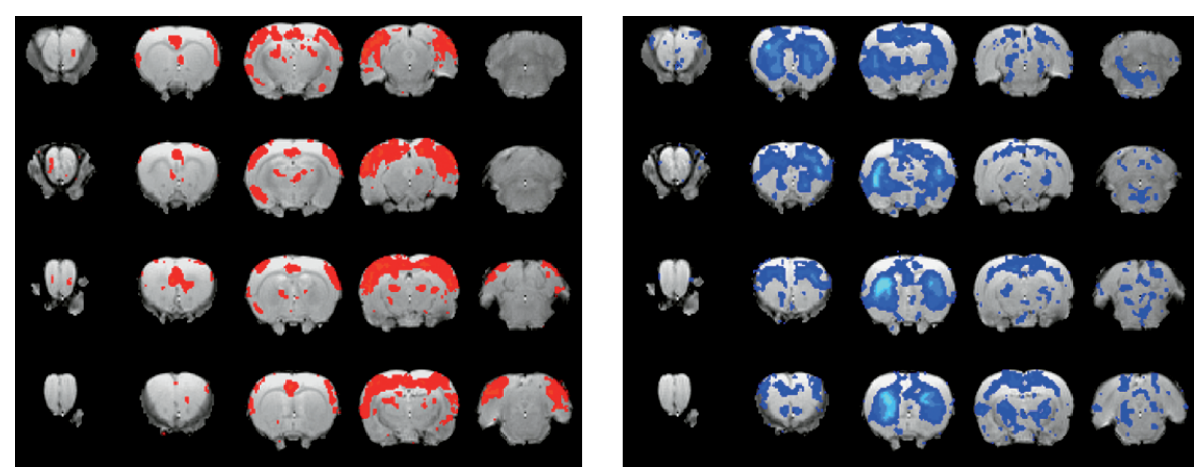

Figure 5. Statistical activation maps calculated using the regressors derived from FSL analyses. In shamoperated animals, positive pilocarpine-induced CBV changes were found in the cortex (on left) whereas negative pilocarpine-induced CBV changes were found in the striatum (on right). 
Figures $6 \mathrm{a}$ and $6 \mathrm{~b}$ show the calculated activation maps based on applying the FSL regressors for the various experimental groups. Compared to sham operation, positive activation responses were more pronounced after left-hemisphere infarction and at 1 week after right-hemisphere infarction, particularly in the lateral cortex, rostromedial hippocampus and thalamus (Figure 6a). At 3 weeks after right-hemipshere infarction, positive activation responses were markedly reduced in the cortex. A similar picture was observed for the negative activation in the striatum (Figure 6b), where negative activation responses were found to be more pronounced after righthemisphere infarction and at 1 week after left-hemisphere infarction, particularly in the rostromedial cortex, hippocampus and thalamus. Negative activation responses in the striatum were diminished as compared to sham-operation, most notably at 3 weeks after left-hemisphere infarction.

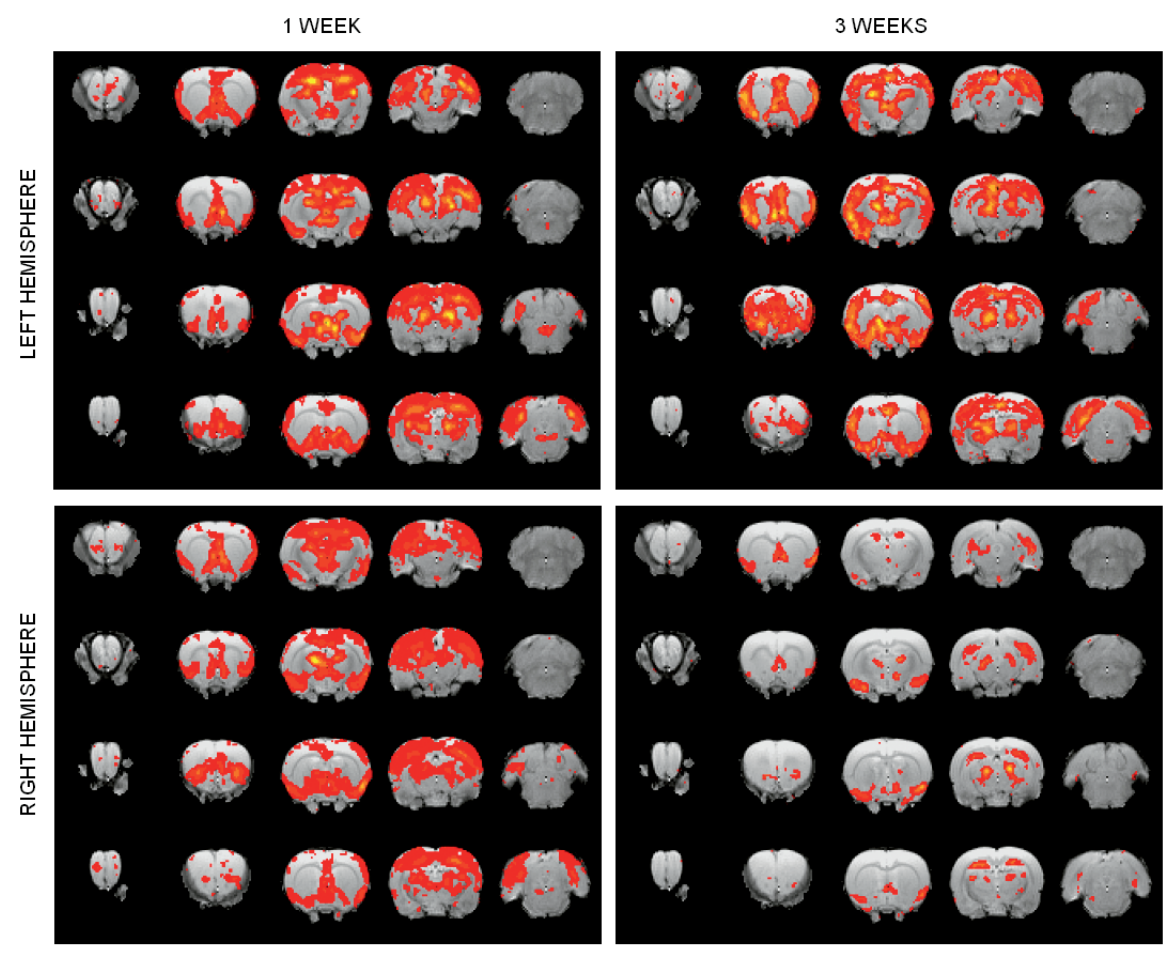

Figure 6a. Statistical activation maps using the regressor for positive pilocarpine-induced CBV response at 1 week and 3 weeks after left hemisphere infarction (top row) and after right hemisphere infarction (bottom row). 

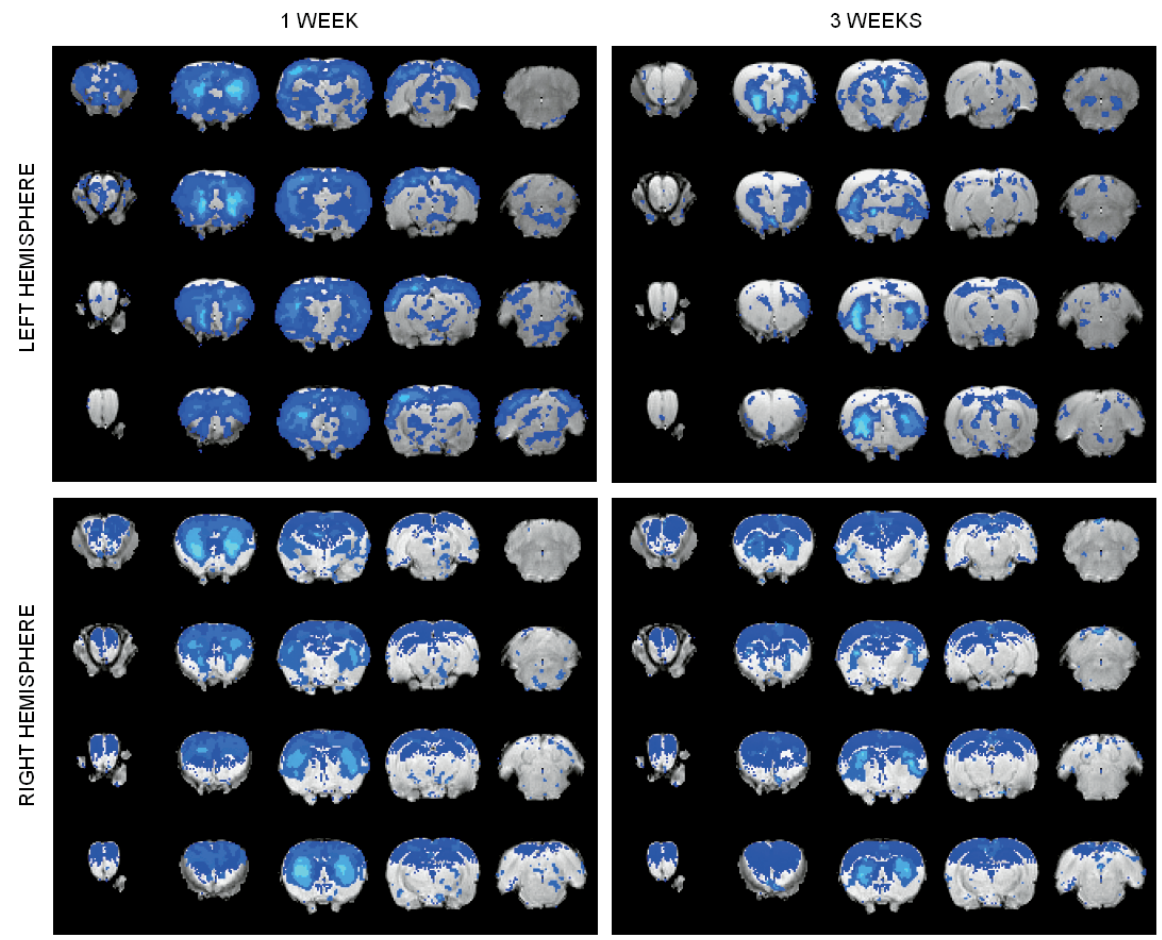

Figure 6b. Statistical activation maps using the regressor for negative pilocarpine-induced CBV response at 1 week and 3 weeks after left hemisphere infarction (topr row) and after right hemisphere infarction (bottom row). 


\section{Experiment II - Immunohistochemistry of cholinergic cells}

We refer to our previous publication for histological features of unilateral frontal cortex infarction [7]. Lesion volume did not differ between left-hemisphere and righthemisphere lesion groups (respectively, $1.27 \pm 0.18 \mathrm{~mm} 3$ vs. $1.45 \pm 0.45 \mathrm{~mm} 3$; $\mathrm{p}=0.72$, ns); none of the sham-operated animals had a lesion.

Table 1, left, shows the total number of cholinergic cells in the various ROls. No significant differences were found between the lesion groups versus sham-operation for cholinergic cell number in the medial septum (Ch1), diagonal band (Ch2/3) or basal nucleus (Ch4), neither in the ipsilesional nor the contralesional hemispheres. The volume of the ROls did not differ between hemispheres or between experimental groups.

Table 1, right, shows the estimated volume of cholinergic cell bodies in the various ROls. No significant differences were found between the lesion groups versus sham-operation for cell body volume in areas Ch1-4, neither in the ipsilesional nor the contralesional hemispheres.

Table 1. Total cell number and estimated cell volume of cholinergic cells in the various regions-of-interest: medial septum (Ch1), diagonal band (Ch2/3) and basal nucleus (Ch4).

\begin{tabular}{|c|c|c|c|c|}
\hline & & sham & right frontal & left frontal \\
\hline \multicolumn{5}{|c|}{ Total cell number } \\
\hline \multirow[t]{2}{*}{ Ch1 } & right & $420 \pm 33$ & $439 \pm 22$ & $464 \pm 19$ \\
\hline & left & $401 \pm 48$ & $444 \pm 22$ & $463 \pm 13$ \\
\hline \multirow[t]{2}{*}{$\mathrm{Ch} 2 / 3$} & right & $649 \pm 85$ & $587 \pm 40$ & $742 \pm 30$ \\
\hline & left & $681 \pm 95$ & $605 \pm 59$ & $842 \pm 68$ \\
\hline \multirow[t]{2}{*}{ Ch4 } & right & $612 \pm 29$ & $614 \pm 25$ & $645 \pm 21$ \\
\hline & left & $582 \pm 55$ & $659 \pm 69$ & $599 \pm 30$ \\
\hline \multicolumn{5}{|c|}{ Estimated cell volume } \\
\hline \multirow[t]{2}{*}{ Ch1 } & right & $1037 \pm 55$ & $1102 \pm 129$ & $889 \pm 58$ \\
\hline & left & $989 \pm 81$ & $1113 \pm 123$ & $893 \pm 76$ \\
\hline \multirow[t]{2}{*}{$\mathrm{Ch} 2 / 3$} & right & $1255 \pm 89$ & $1496 \pm 192$ & $1420 \pm 71$ \\
\hline & left & $1359 \pm 46$ & $1277 \pm 99$ & $1455 \pm 31$ \\
\hline \multirow[t]{2}{*}{ Ch4 } & right & $1750 \pm 60$ & $1708 \pm 157$ & $1868 \pm 61$ \\
\hline & left & $1703 \pm 59$ & $1691 \pm 97$ & $1829 \pm 55$ \\
\hline
\end{tabular}




\section{DISCUSSION}

Human vascular cognitive impairment has been related to dysfunction of the central cholinergic system. The present experiments investigated responses of the cholinergic system to focal cerebral infarction in rat brain by examining muscarinic receptor activation and cholinergic cell number after photothrombotic infarction to the frontal cortex.

First, we performed single-event phMRI to assess cerebral muscarinic receptor activation at 1 and 3 weeks after photothrombotic infarction of either the left or right frontal cortex. These time-points were chosen on the basis of previously conducted behavioural studies that produced differential effects in cognitive performance [7, 8]. $\mathrm{ROI}$ analysis of the relative CBV changes in the ipsilesional hemisphere showed reductions in muscarinic receptor activation inside the ischemic area at 1 and 3 weeks after right-sided cortical infarction, although these reductions were not statistically significant. Significant activation changes were detected at 3 weeks after righthemisphere infarction in the sensory cortex as well as striatum distant from the ischemic lesion. In contrast, statistical activation maps calculated in FSL showed more pronounced cortical activation in all lesion groups except for right-hemisphere infarction at 3 weeks. Furthermore, the statistical maps showed a negative activation response in the cortex at 1 week in both lesion groups and at 3 weeks after rightsided infarction.

The apparent mismatch between the results of ROI analysis versus FSL analysis can be explained as follows: 1) ROI analysis compares mean MR signal changes between experimental groups whereas FSL analysis is based on a statistical fitting of two probabilistic signal components deduced from sham-operated animals to the various lesion groups; 2) the cortical areas with reduced $\mathrm{mAChR}$ activation in the ROI analysis are different from the areas showing increased cortical activation in the FSL analysis. We lack a sound explanation for the observation that the two probabilistic components chosen from FSL analysis (cortex and striatum) showed several other positive and negative activation responses as compared to sham operated animals in areas such as hippocampus and thalamus.

Our phMRI findings corroborate previous ex vivo observations using immunohistrochemistry and autoradiography that have shown alterations in muscarinic acetylcholine receptor ( $\mathrm{mAChR}$ ) number and $\mathrm{mAChR}$ binding after brain ischemia in rats. Using different models of global and focal subcortical ischemia, Mizobuchi et al. [15] found decreased numbers of $m A C h R$ and increased $m A C h R$ binding affinity inside ischemic areas, whilst subsequent reperfusion caused the number of $m A C h R$ to increase. These mAChR changes occurred within hours after induction of ischemia, 
whereas significant decreases in cholinergic cell number in the hippocampus were only found after a few days. Barbelivien et al. observed reduced muscarinic subtype M1 receptor density in the ipsilateral cortex and basal nucleus following an ischemic insult to the frontoparietal cortex by permanent distal middle cerebral artery occlusion (MCAO) [11]. Alterations in mAChR binding have also been shown after transient forebrain ischemia [12]. Nagasawa et al. [14] have shown decreased $m A C h R$ binding in the ipsilateral cerebral cortex and lateral segment of the caudate putamen after transient MCAO followed by 3 days of recirculation. Moreover, several days after ischemia significant decreases were observed in areas that had not been directly affected by the original ischemic insult. Interestingly, an asymmetry in mAChR binding sites and distribution of $\mathrm{mAChR}$ subtypes has been described, in which the right cerebral cortex shows higher receptor densities compared to the left [27]. In addition, it was shown that the down-regulation process of muscarinic receptors is more active in the right than in the left cerebral cortex of the rat. This may explain why in our study reduced muscarinic receptor activation was found particularly after righthemisphere infarction.

Second, we determined the number and volume of ChAT-immunopositive cells in several basal forebrain nuclei. We did not find a significant alteration in cholinergic cell number or cell body volume following focal infarction to the right or left frontal cortex. Concerning putative retrograde changes in basal forebrain neurons of the cerebral cholinergic system, our study corroborates previous findings in rats, in which secondary cell death was not observed in the basal nucleus after lesioning its target area, the cerebral cortex $[9,28]$. Unilateral cortical damage has instead been shown to cause cellular swelling followed by permanent shrinkage of basal nucleus cholinergic neurons ipsilateral to the lesion [9, 29-31]. Subcortical ischemia induced by transient MCAO, however, has been reported to cause delayed neuronal degeneration in ischemic foci as well as remote areas [14]. Since large portions of the cholinergic system were lesioned in these studies, our localized photothrombotic cortical lesion may have been too small to induce significant alterations in basal forebrain cell structure.

In contrast to the absence of structural degeneration of cholinergic cells in the basal forebrain, our phMRI results demonstrate considerable functional changes within the cholinergic system. Several lines of evidence have indicated that the central cholinergic system is sensitive to ischemia, and even mild hypoxia may impair the synthesis of acetylcholine (for review see: [32]). Cholinergic receptors are particularly involved in the control of cerebral blood flow, by mediating vasodilatation of cortical microvessels upon stimulation of cholinergic cells in the basal forebrain. The importance of these receptors is underscored by our observations showing widespread 
changes in cortical activation, both inside and distant from an ischemic territory. Vascular cognitive impairment may therefore be partly related to an ischemia-induced breakdown in cholinergic neurogenic control of cerebral blood flow [33]. In humans, functional neuroimaging has recently been applied to measure changes in regional brain activation during cognitive task performance after pharmacologic manipulation. In patients with Alzheimer's disease and mild cognitive impairment, recent reports have demonstrated alterations in brain activation after acute and prolonged administration of acetylcholinesterase inhibitors [34-39]. Comparable studies with respect to human vascular cognitive impairment, however, are lacking.

In conclusion, we have shown changes in muscarinic receptor activation without degeneration of cholinergic cells after a relatively small infarction of the cerebral cortex in rat brain. Functional imaging of muscarinic receptor activation may therefore be an interesting tool for future studies on the diagnosis and therapeutic evaluation of vascular cognitive impairment. 


\section{REFERENCES}

1. Roman GC. Facts, myths, and controversies in vascular dementia. J Neurol Sci. 2004 Nov 15;226(12):49-52.

2. Erkinjuntti T. Cognitive decline and treatment options for patients with vascular dementia. Acta Neurol Scand Suppl. 2002;178:15-8.

3. Kalaria RN, Ballard C. Stroke and cognition. Curr Atheroscler Rep. 2001 Jul;3(4):334-9.

4. Erkinjuntti T. Diagnosis and management of vascular cognitive impairment and dementia. J Neural Transm Suppl. 2002(63):91-109.

5. Baxter MG, Chiba AA. Cognitive functions of the basal forebrain. Curr Opin Neurobiol. 1999 Apr;9(2):178-83.

6. Everitt BJ, Robbins TW. Central cholinergic systems and cognition. Annu Rev Psychol. 1997;48:649-84.

7. Hoff El, Blokland A, Rutten K, Steinbusch HW, van Oostenbrugge RJ. Dissociable effects in reaction time performance after unilateral cerebral infarction: a comparison between the left and right frontal cortices in rats. Brain Res. 2006 Jan 19;1069(1):182-9.

8. Hoff El, van Oostenbrugge RJ, Liedenbaum M, Steinbusch HW, Blokland A. Effects of right-hemisphere cortical infarction and muscarinic acetylcholine receptor blockade on spatial visual attention performance in rats. Behav Brain Res. 2007 Mar 12;178(1):62-9.

9. Sofroniew MV, Pearson RC, Powell TP. The cholinergic nuclei of the basal forebrain of the rat: normal structure, development and experimentally induced degeneration. Brain Res. 1987 May 19;411(2):31031.

10. Salmeron BJ, Stein EA. Pharmacological applications of magnetic resonance imaging. Psychopharmacol Bull. 2002 Winter;36(1):102-29.

11. Barbelivien A, Vaussy C, Marchalant $\mathrm{Y}$, Maubert E, Bertrand N, Beley A, et al. Degeneration of the basalocortical pathway from the cortex induces a functional increase in galaninergic markers in the nucleus basalis magnocellularis of the rat. J Cereb Blood Flow Metab. 2004 Nov;24(11):1255-66.

12. Waller SB, Nyberg $P$, Dietz NJ. Temporal profile of neurochemical recovery following injury by transient cerebral ischemia. J Gerontol A Biol Sci Med Sci. 1995 Sep;50(5):B307-14.

13. Dawson VL, Hsu CY, Liu TH, Dawson TM, Wamsley JK. Receptor alterations in subcortical structures after bilateral middle cerebral artery infarction of the cerebral cortex. Exp Neurol. 1994 Jul;128(1):88-96.

14. Nagasawa H, Araki T, Kogure K. Alteration of muscarinic acetylcholine binding sites in the postischemic brain areas of the rat using in vitro autoradiography. J Neurol Sci. 1994 Jan;121(1):27-31.

15. Mizobuchi $\mathrm{H}$. [Changes in muscarinic cholinergic receptor and choline acetyltransferase in experimental ischemic brain]. Nippon Geka Hokan. 1989 Jan 1;58(1):93-106.

16. Hoff El, Oude Egbrink MG, Heijnen VV, Steinbusch HW, van Oostenbrugge RJ. In vivo visualization of vascular leakage in photochemically induced cortical infarction. J Neurosci Methods. 2005 Jan 30;141(1):135-41.

17. Dousset V, Ballarino L, Delalande C, Coussemacq M, Canioni P, Petry KG, et al. Comparison of ultrasmall particles of iron oxide (USPIO)-enhanced T2-weighted, conventional T2-weighted, and gadoliniumenhanced T1-weighted MR images in rats with experimental autoimmune encephalomyelitis. AJNR Am J Neuroradiol. 1999 Feb;20(2):223-7.

18. Mandeville JB, Marota JJ, Kosofsky BE, Keltner JR, Weissleder R, Rosen BR, et al. Dynamic functional imaging of relative cerebral blood volume during rat forepaw stimulation. Magn Reson Med. 1998 Apr;39(4):615-24.

19. Paxinos G, Watson C. The Rat Brain in Stereotaxic Coordinates. 4th edition ed. New York: Academic Press; 1998.

20. Jenkinson $\mathrm{M}$, Smith $\mathrm{S}$. A global optimisation method for robust affine registration of brain images. Med Image Anal. 2001 Jun;5(2):143-56. 
21. Jenkinson M, Bannister $\mathrm{P}$, Brady M, Smith $\mathrm{S}$. Improved optimization for the robust and accurate linear registration and motion correction of brain images. Neuroimage. 2002 Oct;17(2):825-41.

22. Beckmann CF, Smith SM. Probabilistic independent component analysis for functional magnetic resonance imaging. IEEE Trans Med Imaging. 2004 Feb;23(2):137-52.

23. Hyvarinen A. Fast and robust fixed-point algorithms for independent component analysis. IEEE Trans Neural Netw. 1999;10(3):626-34.

24. Woolrich MW, Ripley BD, Brady M, Smith SM. Temporal autocorrelation in univariate linear modeling of FMRI data. Neuroimage. 2001 Dec;14(6):1370-86.

25. Vacca LL. Laboratory manual of histochemistry. New York: Raven Press; 1985.

26. Mesulam MM, Mufson EJ, Wainer BH, Levey Al. Central cholinergic pathways in the rat: an overview based on an alternative nomenclature (Ch1-Ch6). Neuroscience. 1983 Dec;10(4):1185-201.

27. Pediconi MF, Roccamo de Fernandez AM, Barrantes FJ. Asymmetric distribution and down-regulation of the muscarinic acetylcholine receptor in rat cerebral cortex. Neurochem Res. 1993 May;18(5):56572.

28. Sophou S, Dori I, Antonopoulos J, Parnavelas JG, Dinopoulos A. Apoptosis in the rat basal forebrain during development and following lesions of connections. Eur J Neurosci. 2006 Jul;24(2):573-85.

29. Liberini P, Pioro EP, Maysinger D, Cuello AC. Neocortical infarction in subhuman primates leads to restricted morphological damage of the cholinergic neurons in the nucleus basalis of Meynert. Brain Res. 1994 Jun 13;648(1):1-8.

30. Sofroniew MV, Pearson RC, Eckenstein F, Cuello AC, Powell TP. Retrograde changes in cholinergic neurons in the basal forebrain of the rat following cortical damage. Brain Res. 1983 Dec 19;289(12):370-4.

31. Sofroniew MV, Pearson RC. Degeneration of cholinergic neurons in the basal nucleus following kainic or $\mathrm{N}$-methyl-D-aspartic acid application to the cerebral cortex in the rat. Brain Res. 1985 Jul 22;339(1):186-90.

32. Hartig W, Bauer A, Brauer K, Grosche J, Hortobagyi T, Penke B, et al. Functional recovery of cholinergic basal forebrain neurons under disease conditions: old problems, new solutions? Rev Neurosci. 2002;13(2):95-165.

33. Scremin OU, Jenden DJ. Cholinergic control of cerebral blood flow in stroke, trauma and aging. Life Sci. 1996;58(22):2011-8.

34. Venneri A. Imaging treatment effects in Alzheimer's disease. Magn Reson Imaging. 2007 Jul;25(6):95368.

35. Dickerson BC. Functional magnetic resonance imaging of cholinergic modulation in mild cognitive impairment. Curr Opin Psychiatry. 2006 May;19(3):299-306.

36. Gron G, Brandenburg I, Wunderlich AP, Riepe MW. Inhibition of hippocampal function in mild cognitive impairment: targeting the cholinergic hypothesis. Neurobiol Aging. 2006 Jan;27(1):78-87.

37. Goekoop R, Scheltens P, Barkhof F, Rombouts SA. Cholinergic challenge in Alzheimer patients and mild cognitive impairment differentially affects hippocampal activation--a pharmacological fMRI study. Brain. 2006 Jan;129(Pt 1):141-57.

38. Goekoop R, Rombouts SA, Jonker C, Hibbel A, Knol DL, Truyen L, et al. Challenging the cholinergic system in mild cognitive impairment: a pharmacological fMRI study. Neuroimage. 2004 Dec;23(4):14509.

39. Rombouts SA, Barkhof F, Van Meel CS, Scheltens P. Alterations in brain activation during cholinergic enhancement with rivastigmine in Alzheimer's disease. J Neurol Neurosurg Psychiatry. 2002 Dec;73(6):665-71 

Chapter

General discussion 
Chapter 7 
Vascular pathology is increasingly recognized as a common cause of cognitive impairment in the elderly. Vascular cognitive impairment ( $\mathrm{VCl})$ is a heterogeneous group of cognitive disorders that share a presumed vascular cause. It is associated with large vessel disease, with small vessel disease including subcortical ischemic vascular disease, and with non-infarct ischemic changes [1]. $\mathrm{VCl}$ can potentially be prevented or the course of cognitive decline ameliorated. One of the suggested treatment options has been modulation of the central cholinergic system [2], which is known to play a major role in cognitive performance. The present thesis explored the putative relationship between the development of $\mathrm{VCl}$ and damage to the central cholinergic system in an animal model of cerebral infarction. To this end, cortical infarction was produced in rat brain by application of the photothrombotic stroke model that allowed for the investigation of: (1) cognitive effects without confounding by gross sensorimotor deficits and (2) local as well as remote changes to the cholinergic system.

\section{Vascular cognitive impairment: behavioural analysis in rat brain}

In the first part of this thesis, the objective was to establish an experimental equivalent of human $\mathrm{VCl}$. Rodents are the most commonly employed animals to model human cognitive function. Much of the scepticism towards rat models arises from the expectation that neurological symptoms should have the same manifestation across species [3]. The modelling of human-like symptoms in animals should however be made primarily on the basis of an expectation of functional similarity, rather than on one of physical identity. The use of such a comparative approach allows the assessment of cognitive processes in rats which appear to be activated in humans when performing cognitive tasks. Advances have been made in identifying rat equivalents of neurological deficits seen in human diseases such as Parkinson's disease, traumatic brain injury and ischemic stroke. Both stroke and traumatic brain injury can produce focal and widespread secondary damage in the brain, which can yield acute and chronic impairments of sensory, motor and cognitive functions. The focus on neuroprotection and recovery of motor function in clinical stroke research has been replaced over the last decade by a view incorporating $\mathrm{VCI}$. In parallel, the importance of behavioural outcome measures in addition to histological assessment has become increasingly recognized in experimental stroke research [4]. Commonly used histological techniques, such as counts of residual neurons or determinations of infarct lesion volume, cannot detect subtle cellular alterations that might cause neuronal dysfunction. Accordingly, there is ample evidence of dissociation between behavioural and histological endpoints in animal models of stroke [5]. 
The interpretation of complex cognitive data in experimental stroke research can be problematic because stroke is often associated with sensorimotor deficits that may become confounding factors. In our studies such a confounder is not likely. The photothrombotic model produces relatively small cortical lesions. In the reaction time experiments these lesions were located outside of the sensorimotor cortex, and normal behaviour including gross motor function was already observed within hours of photothrombotic infarction. Moreover, motor response latency was measured in the order of milliseconds and did not increase upon infarction suggesting that the execution of movement was unaffected.

The pattern of cognitive deficits in patients with $\mathrm{VCl}$ varies considerably. Patients who have had a stroke, however, are most frequently affected by global impairments in attention and cognition. These general impairments are more closely associated with worse functional outcomes than executive dysfunction and isolated memory deficits. Single strategic infarcts can lead to specific cognitive profiles, whereas subcortical lesions are often associated with abnormalities of information processing speed, executive function, and emotional liability. This cluster of features, termed the subcortical syndrome, can also present with deficits that result from cortical lesions. In accordance with these considerations, the present thesis shows that cortical infarction in rat brain produces two common types of post-stroke cognitive deficits (slowing of information processing speed, impairment of attention performance) observed in the subcortical neuropsychological profile of human VCl. Rather than undermine the extrapolation of experimental studies to human diseases, this shows the phylogenetic comparability of basic cognitive functions across species.

By chance a neglect phenomenon was observed in right hemisphere lesioned rats, both in the reaction time and the attention performance experiments. The syndrome of neglect is defined as an inability to report, respond or orient to stimuli contralateral to a cerebral lesion despite intact elementary sensory or motor function. Such an inability to respond to the contralesional hemispace occurs frequently in stroke patients, particularly in right hemisphere strokes. In rats, right hemisphere infarction can also produce neglect phenomena. There are numerous reports showing that rats subjected to stroke fail to orient toward contralateral stimuli [for review, see [6]]. This failure to respond following stroke can result from sensory inattention, inability to initiate motor responses or a combination of these two components (sensorimotor desintegration). Most experimental stroke studies report hemi-inattention or sensorimotor integration deficits without attempting to dissociate the sensory, motor and motivational components. The present thesis substantiates that frontal cortex infarction in rat brain causes two types of neglect phenomena: (1) a sensorimotor disintegration of the motor preparatory process, and (2) a transient impairment in 
spatial attention of the contralateral visual field. By contrast, parietal infarction causes a non-lateralized impairment of attention. Lateralization or hemispheric dominance of cognitive functions is therefore not exclusively a human trait.

\section{Vascular cognitive impairment: cholinergic analysis in rat brain}

In the second part of this thesis, the objective was to characterize alterations to the central cholinergic system after photothrombotic infarction in rat brain. The central cholinergic system is known to modulate cognitive performance. Most of the cholinergic nuclei are located in the basal forebrain and their axonal projections innervate all layers of the cerebral cortex. These extensive projections mediate fundamental cognitive processes such as the speed of information processing in the cerebral cortex and attention performance by modulating stimulus selection [7]. Although minor differences exist in the neuroanatomy of different species of animals, the main cholinergic pathways that have been found in rat brain are similar to those found in other mammals including humans.

The application of the photothrombotic model described in this thesis resulted in transcortical infarction of all layers of the cortex, whereas additional damage to the corpus callosum was minimal or non-existent. Axonal damage and subsequent anterograde and retrograde degeneration of basal forebrain cholinergic projections are therefore likely to have occurred. However, infarction to the frontal cortex did not result in a diminished cholinergic cell number in the basal forebrain. This suggests that cell bodies are intact and should be capable of regenerative responses through secondary plastic changes. Elaborate reviews by Henderson and Hartig et al. [8,9] state that there is little evidence for regeneration or collateral sprouting of cholinergic axons in the basalo-cortical pathway. The only apparent compensatory response is thought to be upregulation of neurotransmitter function, as the strong muscarinic receptor activation after 1 week of frontal cortex infarction suggests.

To assess possible alterations within the central cholinergic system in vivo, neuroimaging of cholinergic muscarinic receptor function was performed. This showed widespread changes in receptor activation inside the ischemic lesion area as well as in areas distant from infarction, which was found particularly after right frontal cortex infarction. Apparently, cerebral lateralization in the rat involves functional alterations on neurotransmitter receptor level. 


\section{Vascular cognitive impairment: integrative analysis in rat brain}

The present paragraph tries to relate the observed experimental equivalents of human $\mathrm{VCl}$ with the ischemia-induced alterations of the cholinergic system (see Table).

Table. Summary of results: Comparison of behavioural versus neuroimaging studies

\begin{tabular}{llll}
\hline & RTR & SSVA & mAChR \\
\hline Frontal (right) & $\begin{array}{l}<4 \text { weeks: transient deficit in } \\
\text { contralateral motor initiation } \\
\text { (neglect phenomenon) }\end{array}$ & $\begin{array}{l}<1 \text { week: transient deficit contra- } \\
\text { lateral visual attention (neglect } \\
\text { phenomenon) }\end{array}$ & $\begin{array}{l}\text { week 1: high } \\
\text { week 3: very low }\end{array}$ \\
& $>3$ weeks: gradual reaction time & & \\
& increase (mental slowing) & & week 1: very high \\
Frontal (left) & $>3$ weeks: gradual reaction time & N/A & week 3: low \\
& increase (mental slowing) & & N/A \\
Parietal (right) & N/A & $<1$ week: non-lateralized visual & \\
& & attention deficit & \\
\hline
\end{tabular}

* Induced by scopolamine. Abbreviations: RTR = reaction time responding, and SSVA = sustained spatial visual attention (behavioural tasks), $\mathrm{mAChR}=$ muscarinic acetylcholine receptor (pharmacological MRI)

First, a slowing of information processing speed developed gradually over the course of 4 weeks after infarction to the frontal cortex, which was deemed similar to the poststroke mental slowing observed in humans. If related to changes in the cholinergic system, such a gradual slowing of information processing speed may be caused by secondary plastic changes to cholinergic neurons or a down regulation of cholinergic receptors. Since the number of cell bodies was found to be intact, the cholinergic system should be capable of regenerative responses. Indeed, down regulation of muscarinic receptors was shown in this thesis by a diminished cortical activation of muscarinic receptors at 3 weeks after infarction to either the left or right frontal cortex. There is no certain explanation why reduced muscarinic receptor activation was found particularly after right hemisphere infarction, while the general increase in reaction time did not differ between right versus left hemisphere lesioned animals. But as mentioned in Chapter 6, it has been reported that the right cerebral cortex of rat brain contains higher $\mathrm{mAChR}$ densities compared to the left, and that the downregulation process of these receptors is more active in the right cerebral cortex. 
Second, transient deficits in attention performance lasting no more than 1 week after right hemisphere cortical infarction were observed. After parietal infarction, this attention deficit may be related to cholinergic function since a similar non-lateralized attention deficit was found with muscarinic receptor blockade. By contrast, frontal infarction resulted predominantly in a lateralized, contralesional attention deficit that was explained as an attentional impairment for the left visual field, which is in accordance with the most prevalent form of neglect following right hemisphere damage. It remains speculatory whether this neglect phenomenon involves cholinergic function.

Third, the testing of reaction time performance and visual attention performance after right frontal cortex infarction revealed two types of neglect. Phenomena associated with neglect can be interpreted along the spectrum of sensorimotor integration as either reflecting deficits in attentional performance ('sensory neglect') versus intentional performance ('motor neglect'). In accordance with the latter, this thesis reports on a form of motor neglect that lasted 3 to 4 weeks. It was attributed to an impairment of movement initiation that relates well with the functional role of the frontal cortex. Reduced cholinergic function may be involved, since muscarinic receptor activation was reduced at 3 weeks in right hemisphere infarction. However, at 1 week after infarction the neglect phenomenon coincided with an apparent increase in muscarinic activation. The present thesis indicates that neglect after right frontal infarction harbours a short-lived attentional component that may relate to an early reduction in muscarinic receptor function. However, a direct relation between left-sided neglect and cholinergic dysfunction was not proven. Proof would require additional experimentation, for instance with direct cortical injection of muscarinic acetylcholine receptor antagonists. Nonetheless, it is clearly shown that spatial visual attention is impaired after cortical infarction as well as after cholinergic blockade.

And last, it is likely that the observed behavioural deficits may not be related solely to alterations of the cholinergic system. Apart from acetylcholine, several other neurotransmitter systems are known to play a critical role in performance of cognitive tasks following brain damage [10]. Among these neurotransmitters are dopamine, noradrenaline, and glutamate. It is evident that each of these neurotransmitter systems contributes to a range of different cognitive functions, and that many of these functions are dependent on the action of multiple neuronal networks. Additionally, it is possible that some of the putative cognitive effects of these neurotransmitters are secondary to their effects on other cognitive systems. For example, an effect on memory tasks may arise from modulation of arousal or attention. The large range of functions in which these transmitters are implicated is reflected anatomically in their wide distribution across many different brain regions. 


\section{Future perspectives}

The photothrombotic stroke model itself has relatively limited significance in unravelling the pathophysiology of cerebral ischemia or infarction, because the development of tissue damage is very rapid and without reperfusion or collateral blood flow. As discussed above, however, photothrombotic infarction of the cerebral cortex in rat brain does offer a means to induce certain types of behavioural effects that are observed in human VCl. Additionally, it might be helpful in the development of new therapeutic regimens targeting $\mathrm{VCl}$. In this thesis it is demonstrated that pharmacological MRI with pilocarpine offers the possibility to study central cholinergic function through the activation of muscarinic receptors. Such a neuroimaging method could be used to detect possible cholinergic changes in cognitively impaired stroke patients qualitatively and quantitatively, and be used in the follow-up of patients with VCl. Furthermore, intervention studies with cholinergic medication in stroke patients could be monitored. The next two paragraphs discuss the potential for functional neuroimaging and neuropharmacological modulation in $\mathrm{VCl}$.

\section{Functional neuroimaging in vascular cognitive impairment}

Magnetic resonance imaging (MRI) provides a non-invasive and multimodal tool to study neurological disorders in experimental models [11]. Routine anatomical neuroimaging detects cortical and subcortical infarcts, haemorrhage, white matter changes and gliosis. No current brain imaging techniques are capable of predicting the clinical presentation of $\mathrm{VCl}$ in stroke patients. The use of novel functional neuroimaging studies might help to assess pathophysiological processes, estimate the prognosis of functional outcome and evaluate the efficiency of treatment strategies after stroke $[12,13]$. A number of novel ways of using MRI to visualize the action of drugs on animal and human brain i.e. phMRI are becoming established tools in translational psychopharmacology. Using drugs with known pharmacology it is possible to investigate how neurotransmitter systems are involved in neural systems engaged by other processes, such as cognitive challenge (modulation phMRI) or to examine the acute effects of the drug itself in the brain (challenge phMRI) [14].

Pharmacological functional MRI of cholinergic receptor activation may be developed into a method whereby stroke patients can be selected to benefit from psychological and medical intervention. Our results demonstrated that ischemic brain damage produces widespread functional changes in the cholinergic system. We have shown that cholinergic receptor function can be measured in vivo, and therefore, phMRI can be used in a disease model to assess the integrity of neurotransmitter 
receptor function. Thus far, phMRI studies in rats have mainly involved the characterization of drug effects on regional cerebral activation in normal rats [15]. By contrast, human phMRI studies investigating the cholinergic system have primarily combined drug administration with a cognitive task in order to investigate drug modulation of the BOLD response to a cognitive challenge [16-21]. Pharmacological imaging of the cholinergic system could be developed for use in the diagnostic workup or evaluation of cognitive disorders such as VCl. Stroke patients with and without cognitive impairment could then be tested for reduced cholinergic function. Ultimately, the effect of treatment in $\mathrm{VCl}$ could be monitored.

\section{Pharmacological modulation of the cholinergic system after brain ischemia}

Several studies have investigated the use of cholinesterase inhibitors on functional outcome after experimental stroke in rats. Zhao et al [22] did not find a beneficial effect of galantamine on motor recovery or spatial learning, administered prior to and following photothrombotic infarction of the motor cortex. By contrast, lliev et al [23] showed a beneficial effect of galantamine on the recovery of learning ability when administered once only after transient forebrain ischaemia, suggesting a direct effect on the early pathologic mechanisms of central nervous system damage. Moreover, Borlongan et al. [24] showed that methanesulfonyl-fluoride attenuated deficits in learning and memory following focal cerebral ischemia, possibly by preserving a functional cholinergic system.

It may be possible to target cognitive protection in the acute human stroke setting. A review by Parton et al. [10] discusses several studies that describe modulation of the cholinergic system in humans. Partial protection of the cholinergic system after ischemic injury has been reported by the use of cholinesterase inhibitors. Such treatment could help to preserve the cholinergic system after human brain injury, with the window for the initiation of treatment potentially being much larger than for current therapies. It may also be possible to pharmacologically treat established and chronic cognitive deficits. As with acute intervention, there has been considerable interest in the use of cholinesterase inhibitors due to the significance of the cholinergic system in learning, memory and arousal. Controlled clinical trials with donepezil, galantamine and rivastigmine in $\mathrm{VCl}$ have demonstrated improvements in cognition, behaviour and activities of daily living [25]. Limited data are available when considering the impact of galantamine on $\mathrm{VCl}$, and there is some evidence of benefit of rivastigmine in $\mathrm{VCl}$. Available evidence supports the benefit of donepezil in improving cognition, clinical global impression and activities of daily living in patients with probable or possible mild to moderate $\mathrm{VCl}$ after 6 months treatment. 
As a result, there is generally limited enthusiasm for pharmacological intervention with cholinomimetic drugs in stroke patients with VCl. Other suggested therapies such as cognitive rehabilitation for memory deficits or spatial neglect following stroke have also failed to show clear evidence of effectiveness [26,27]. But a treatment strategy combining neuropharmacological intervention with cognitive rehabilitation may be of interest. In addition, pharmacological MRI of cholinergic function may be helpful in selecting patients that may benefit from such a combined treatment approach.

\section{Concluding remarks}

In the present thesis, the putative relationship between the development of $\mathrm{VCl}$ and damage to the central cholinergic system was explored in an animal model of cerebral cortical infarction. First, an experimental equivalent of human $\mathrm{VCl}$ was established in the rat. Second, functional instead of structural changes in the central cholinergic system were shown to occur in rat brain by using phMRI. This neuroimaging method may be developed into a useful tool to detect central cholinergic impairment and evaluate cholinomimetic treatment in $\mathrm{VCl}$. 


\section{REFERENCES}

1. Moorhouse, P. and Rockwood, K. (2008) Vascular cognitive impairment: current concepts and clinical developments. Lancet Neurol, 7, 246-55.

2. Erkinjuntti, T., Roman, G. and Gauthier, S. (2004) Treatment of vascular dementia--evidence from clinical trials with cholinesterase inhibitors. J Neurol Sci, 226, 63-6.

3. Cenci, M.A., Whishaw, I.Q. and Schallert, T. (2002) Animal models of neurological deficits: how relevant is the rat? Nat Rev Neurosci, 3, 574-9.

4. DeVries, A.C., Nelson, R.J., Traystman, R.J. and Hurn, P.D. (2001) Cognitive and behavioral assessment in experimental stroke research: will it prove useful? Neurosci Biobehav Rev, 25, 325-42.

5. Corbett, D. and Nurse, S. (1998) The problem of assessing effective neuroprotection in experimental cerebral ischemia. Prog Neurobiol, 54, 531-48.

6. Reep, R.L., Corwin, J.V., Cheatwood, J.L., Van Vleet, T.M., Heilman, K.M. and Watson, R.T. (2004) A rodent model for investigating the neurobiology of contralateral neglect. Cogn Behav Neurol, 17, 191-4.

7. Sarter, M. and Parikh, V. (2005) Choline transporters, cholinergic transmission and cognition. Nat Rev Neurosci, 6, 48-56.

8. Henderson, Z. (1996) Responses of basal forebrain cholinergic neurons to damage in the adult brain. Prog Neurobiol, 48, 219-54.

9. Hartig, W., Bauer, A., Brauer, K., Grosche, J., Hortobagyi, T., Penke, B., Schliebs, R. and Harkany, T. (2002) Functional recovery of cholinergic basal forebrain neurons under disease conditions: old problems, new solutions? Rev Neurosci, 13, 95-165.

10. Parton, A., Coulthard, E. and Husain, M. (2005) Neuropharmacological modulation of cognitive deficits after brain damage. Curr Opin Neurol, 18, 675-80.

11. Dijkhuizen, R.M. (2006) Application of magnetic resonance imaging to study pathophysiology in brain disease models. Methods Mol Med, 124, 251-78.

12. Dijkhuizen, R.M. and Nicolay, K. (2003) Magnetic resonance imaging in experimental models of brain disorders. J Cereb Blood Flow Metab, 23, 1383-402.

13. Heiss, W.D., Thiel, A., Winhuisen, L., Muhlberger, B., Kessler, J. and Herholz, K. (2003) Functional imaging in the assessment of capability for recovery after stroke. J Rehabil Med, 27-33.

14. Anderson, I.M., McKie, S., Elliott, R., Williams, S.R. and Deakin, J.F. (2008) Assessing human 5-HT function in vivo with pharmacoMRI. Neuropharmacology, 55, 1029-37.

15. Steward, C.A., Marsden, C.A., Prior, M.J., Morris, P.G. and Shah, Y.B. (2005) Methodological considerations in rat brain BOLD contrast pharmacological MRI. Psychopharmacology (Berl).

16. Dickerson, B.C. (2006) Functional magnetic resonance imaging of cholinergic modulation in mild cognitive impairment. Curr Opin Psychiatry, 19, 299-306.

17. Goekoop, R., Scheltens, P., Barkhof, F. and Rombouts, S.A. (2006) Cholinergic challenge in Alzheimer patients and mild cognitive impairment differentially affects hippocampal activation--a pharmacological fMRI study. Brain, 129, 141-57.

18. Saykin, A.J., Wishart, H.A., Rabin, L.A., Flashman, L.A., McHugh, T.L., Mamourian, A.C. and Santulli, R.B. (2004) Cholinergic enhancement of frontal lobe activity in mild cognitive impairment. Brain, 127, 1574-83.

19. Honey, G. and Bullmore, E. (2004) Human pharmacological MRI. Trends Pharmacol Sci, 25, 366-74.

20. Thiel, C.M. (2003) Cholinergic modulation of learning and memory in the human brain as detected with functional neuroimaging. Neurobiol Learn Mem, 80, 234-44.

21. Rombouts, S.A., Barkhof, F., Van Meel, C.S. and Scheltens, P. (2002) Alterations in brain activation during cholinergic enhancement with rivastigmine in Alzheimer's disease. J Neurol Neurosurg Psychiatry, $73,665-71$. 
22. Zhao, C.S., Puurunen, K., Schallert, T., Sivenius, J. and Jolkkonen, J. (2005) Effect of cholinergic medication, before and after focal photothrombotic ischemic cortical injury, on histological and functional outcome in aged and young adult rats. Behav Brain Res, 156, 85-94.

23. Iliev, A.I., Traykov, V.B., Mantchev, G.T., Stoykov, I., Prodanov, D., Yakimova, K.S. and Krushkov, I.M. (2000) A post-ischaemic single administration of galanthamine, a cholinesterase inhibitor, improves learning ability in rats. J Pharm Pharmacol, 52, 1151-6.

24. Borlongan, C.V., Sumaya, I.C. and Moss, D.E. (2005) Methanesulfonyl fluoride, an acetylcholinesterase inhibitor, attenuates simple learning and memory deficits in ischemic rats. Brain Res, 1038, 50-8.

25. Kavirajan, H. and Schneider, L.S. (2007) Efficacy and adverse effects of cholinesterase inhibitors and memantine in vascular dementia: a meta-analysis of randomised controlled trials. Lancet Neurol, 6, 782-92.

26. Bowen, A. and Lincoln, N.B. (2007) Cognitive rehabilitation for spatial neglect following stroke. Cochrane Database Syst Rev, CD003586.

27. Nair, R.D. and Lincoln, N.B. (2007) Cognitive rehabilitation for memory deficits following stroke. Cochrane Database Syst Rev, CD002293. 


\section{Pharmacological magnetic} resonance imaging of muscarinic acetylcholine receptor activation after subcortical ischemia in rat brain

Erik I. Hoff

Robert J. van Oostenbrugge

Wim M. Otte

Jet $P$. van der Zijden

Harry W.M. Steinbusch

Rick M. Dijkhuizen 


\section{ABSTRACT}

Vascular cognitive impairment ( $\mathrm{VCl})$ is defined as cognitive impairment of any severity associated with cerebrovascular disease. Cholinergic deficits are thought to contribute to vascular cognitive impairment. After stroke, these cholinergic deficits may result from altered cholinergic receptor function due to ischemic damage of basal forebrain cholinergic nuclei or their axonal projections. The present proof-of-principle study aimed to detect changes in muscarinic receptor activation following subcortical stroke by use of pharmacological magnetic resonance imaging (phMRI) in rat brain. To this end, phMRI was performed 14 days after transient unilateral middle cerebral artery occlusion (MCAO) in rat brain. Our data demonstrate the feasibility to detect reduced muscarinic receptor activation inside ischemic territory as well as outside the lesion area, suggestive of remote functional impairment. The latter might be due to anterograde damage to cortically projecting cholinergic neurons resulting in alterations in muscarinic receptor function. 


\section{INTRODUCTION}

Vascular cognitive impairment ( $\mathrm{VCl}$ ) is defined as cognitive impairment of any severity associated with cerebrovascular disease [1]. Cholinergic deficits are thought to contribute to $\mathrm{VCl}$ [2]. The putative cholinergic deficits of $\mathrm{VCl}$ may result from altered cholinergic receptor function due to ischemic damage of either basal forebrain cholinergic nuclei or their axonal projections. Studies using autoradiographic analysis or immunohistochemistry have shown decreased muscarinic receptor binding activity and cholinergic fiber density after global and focal cerebral ischemia in rats [3-6].

The aim of the present study was to investigate the feasibility of pharmacological magnetic resonance imaging (phMRI) to detect local and remote changes in muscarinic receptor activation following subcortical ischemic damage in rats. We hypothesized that subcortical ischemia would result in damage to central cholinergic nuclei and/or its projections, and thereby, reduced muscarinic receptor activation in subcortical areas as well as the cerebral cortex.

\section{MATERIALS AND METHODS}

Transient ischemia was performed by 60 -minute right-sided middle cerebral artery occlusion (MCAO) with an intraluminal filament in adult male Wistar rats $[7,8]$. The extent of infarction after 60-min MCAO is variable and may include both subcortical and cortical areas or only subcortical areas. In this present study, four animals with subcortical infarction only were included. We performed contrast-enhanced CBVweighted phMRI in combination with injection of pilocarpine, a non-selective cholinergic muscarinic receptor agonist, to measure the effects on muscarinic receptor activation at 14 days after MCAO.

The MRI experiments were conducted as described elsewhere in this thesis (Chapter 5). In brief, rats were anesthetized with isoflurane and intubated endotracheally. Approximately 60 minutes prior to MRI, the animals were given a subcutaneous injection of $1 \mathrm{mg} / \mathrm{kg}$ scopolamine methyl-bromide (Sigma-Aldrich, Zwijndrecht, The Netherlands) to block peripheral muscarinic effects of pilocarpine injection. T2weighted MRI was performed at 4.7 T (SISCO/Varian systems, Palo Alto, CA, USA) using a spin-echo multi-slice sequence: repetition time $(T R)=3000 \mathrm{~ms}$; echo time $(\mathrm{TE})=20 \mathrm{~ms}$; data matrix $=128 \times 128$ points; field-of-view $(\mathrm{FOV})=35 \times 35 \mathrm{~mm} 2 ; 16$ $x$ 1.2-mm slices. Contrast-enhanced CBV-weighted MRI was performed using a gradient-echo multi-slice sequence: $T R=520 \mathrm{~ms}$; $T E=23 \mathrm{~ms}$; pulse angle $=41^{\circ}$; data matrix $=64 \times 64$ points; FOV $=35 \times 35 \mathrm{~mm} 2 ; 20 \times 1.0-\mathrm{mm}$ slices; temporal resolution $=33 \mathrm{~s}$. Following 10 pre-contrast scans, we intravenously injected ultra- 
small superparamagnetic iron-oxide particles (Sinerem ${ }^{\circledR}$, Guerbet, Aulnay-sous-Bois, France; $10 \mathrm{mg} / \mathrm{kg}$ ). Contrast-enhanced CBV-weighted phMRI was started 15 minutes after injection of the contrast agent. After 10 minutes of baseline measurements (20 scans), pilocarpine was administered and data acquisition continued for 30 minutes thereafter (60 scans).

Data analysis was conducted in three different ways. First, contrast-enhanced CBV-weighted phMRI data were processed and analyzed within the framework of a general linear model (GLM) using SPM2 software package (http://www.fil.ion.ucl.ac.$\mathrm{uk} / \mathrm{spm}$ ). Using the regressors derived previously from CBV-weighted phMRI in methyl-scopolamine pre-treated non-lesioned Lewis rats, we performed a first level or model fitting analysis in SPM2 (see also Chapter 5). Second, we used the Group ICA of fMRI (GIFT) software package (http://icatb.sourceforge. net) to determine up to 20 independent components. Group-level Independent Components Analysis (ICA) has been shown to be a useful method in extracting components of interest from fMRI data [9, 10]. We used the FastICA algorithm [11] using the following algorithm parameters (default): independent components $=20$; autofill data reduction $=$ yes; epsiIon $=0.0001$; iteration $=1000$; approach $=$ defl; $g=$ pow3. Selection of components of interest was based on the following criteria: regional brain activation (sensitivity), no activation of extracranial areas (specificity), and a smooth signal curve shape. Third, relative changes in $\mathrm{CBV}$ were calculated from the contrast-enhanced $\mathrm{CBV}$-weighted MR data as described previously [12]. The CBV response to pilocarpine challenge was analyzed in two regions-of-interest, namely the cerebral cortex and striatum, both ipsilateral and contralateral to the lesioned hemisphere. 


\section{RESULTS}

Positive cortical signal responses in the lesioned hemisphere were largely present in three out of four animals. In one animal, a distinct portion of the ipsilesional parietal cortex did not show an activation response (Figure 1). None of the lesioned animals showed a significant negative response in the ipsilesional striatum. These results were confirmed by independent component analysis, which in none of the animals yielded a negative subcortical component. Moreover, one positive component with a similar partial lack of cortical activation was found in the same one animal [data not shown].

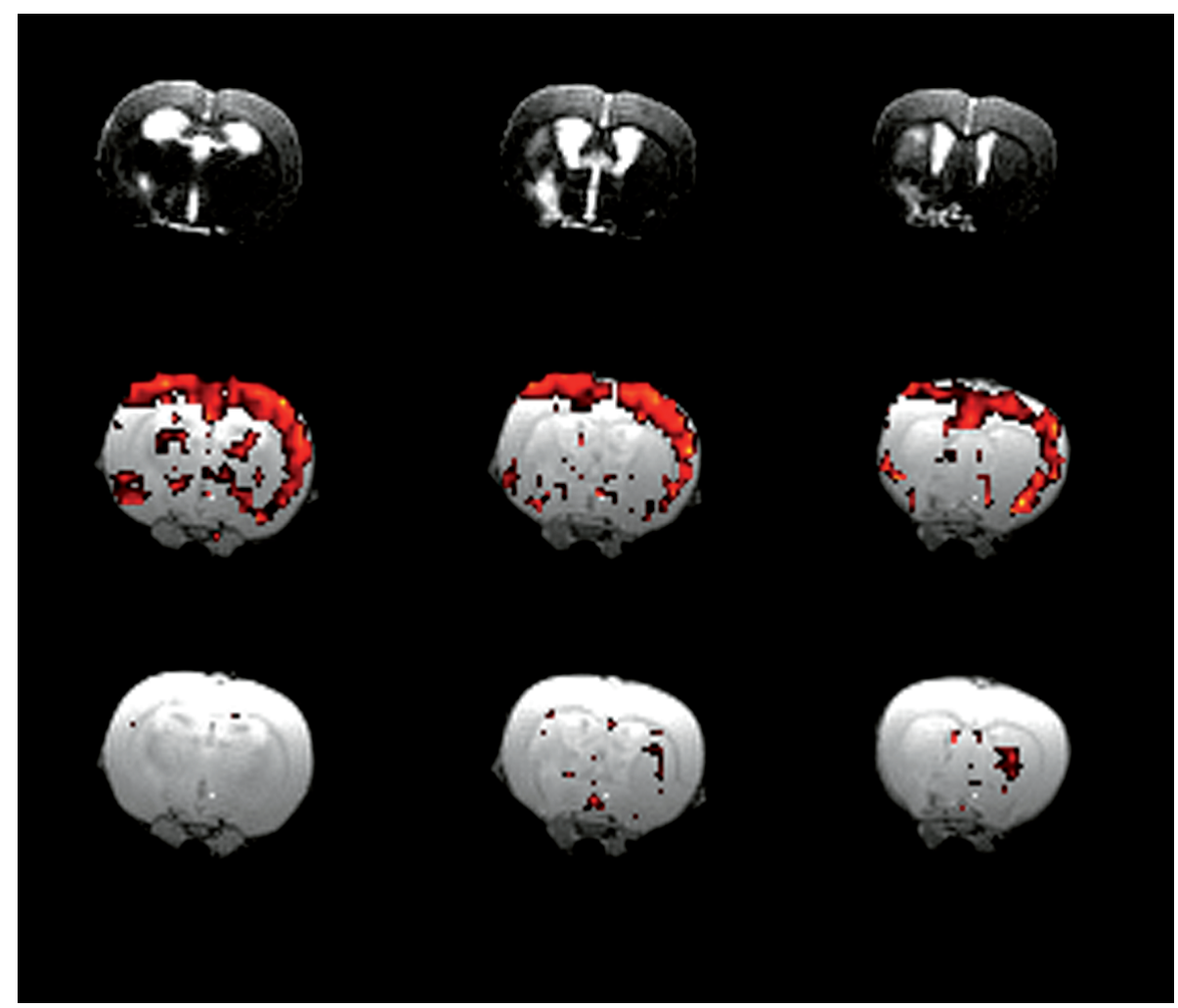

Figure 1. Example of muscarinic activation pattern at two weeks after right-sided MCAO in a rat: Delineation of subcortical ischemic area on $\mathrm{T}_{2}$-weighted MRI (upper images); Statistical maps of significant pilocarpineinduced positive CBV responses overlaid on anatomical MRI images (middle images). Note the loss of activation in large part of the right parietal cortex. 
Figure 2 shows the time-course of pilocarpine-induced CBV changes in the cortex and striatum. In the striatum, the negative CBV response was lower in the lesioned hemisphere as compared to the contralateral hemisphere. In the cortex, the positive CBV response did not differ between hemispheres.

Cortex

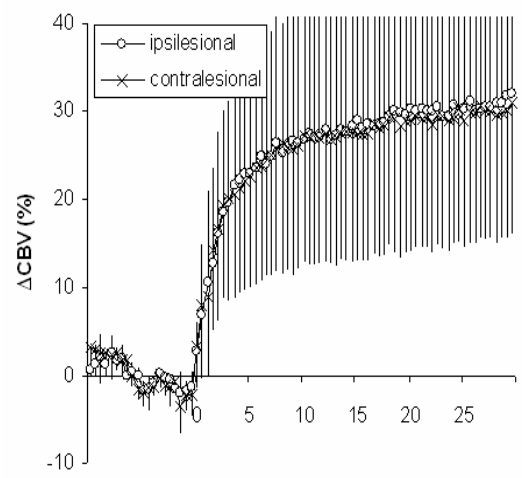

Time after pilocarpine injection ( $\mathrm{min}$ )
Striatum

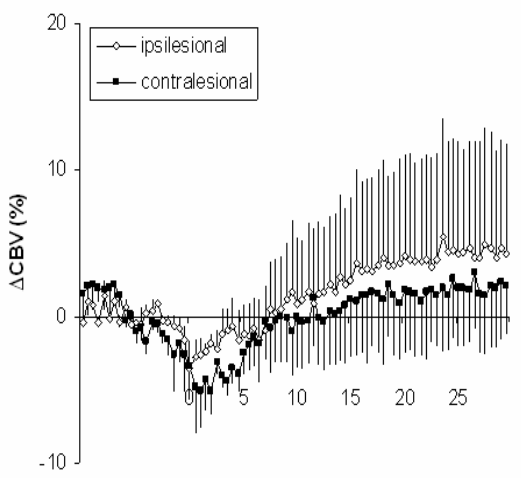

Time after pilocarpine injection ( $\mathrm{min}$ )

Figure 2. Mean CBV change (\%) to pilocarpine challenge in ipsilesional and contralesional striatum and cortex two weeks after unilateral MCAO in rats. Error bars represent standard deviation.

\section{Discussion}

The results of this proof-of-principle study demonstrate the feasibility of phMRI to detect alterations in muscarinic receptor activation inside ischemic territory as well as in the parietal cortex remote from subcortical ischemia in rat brain. Ischemia-induced changes in brain receptor activation by means of phMRI have not been reported previously. Our study corroborates previous autoradiographic findings [13] that imaging of muscarinic receptor function may be of value for the assessment of cholinergic neuron viability following brain ischemia. However, these preliminary findings need to be substantiated in larger series and subsequently developed into a tool suitable for human imaging. Ultimately, functional imaging of muscarinic receptor activation may be used in the diagnosis and therapeutic evaluation of human vascular cognitive impairment. 


\section{REFERENCES}

1. Moorhouse P, Rockwood K. Vascular cognitive impairment: current concepts and clinical developments. Lancet Neurol. 2008 Mar;7(3):246-55.

2. Dichgans M, Markus HS, Salloway S, Verkkoniemi A, Moline M, Wang Q, et al. Donepezil in patients with subcortical vascular cognitive impairment: a randomised double-blind trial in CADASIL. Lancet Neurol. 2008 Apr;7(4):310-8.

3. Mizobuchi $\mathrm{H}$. [Changes in muscarinic cholinergic receptor and choline acetyltransferase in experimental ischemic brain]. Nippon Geka Hokan. 1989 Jan 1;58(1):93-106.

4. Kataoka K, Hayakawa T, Kuroda R, Yuguchi T, Yamada K. Cholinergic deafferentation after focal cerebral infarct in rats. Stroke. 1991 Oct;22(10):1291-6.

5. Nagasawa H, Araki T, Kogure K. Alteration of muscarinic acetylcholine binding sites in the postischemic brain areas of the rat using in vitro autoradiography. J Neurol Sci. 1994 Jan;121(1):27-31.

6. Waller SB, Nyberg P, Dietz NJ. Temporal profile of neurochemical recovery following injury by transient cerebral ischemia. J Gerontol A Biol Sci Med Sci. 1995 Sep;50(5):B307-14.

7. van der Zijden JP, Wu O, van der Toorn A, Roeling TP, Bleys RL, Dijkhuizen RM. Changes in neuronal connectivity after stroke in rats as studied by serial manganese-enhanced MRI. Neuroimage. 2007 Feb 15;34(4):1650-7.

8. Longa EZ, Weinstein PR, Carlson S, Cummins R. Reversible middle cerebral artery occlusion without craniectomy in rats. Stroke. 1989 Jan;20(1):84-91.

9. Calhoun VD, Adali T, Pearlson GD, Pekar JJ. A method for making group inferences from functional MRI data using independent component analysis. Hum Brain Mapp. 2001 Nov;14(3):140-51.

10. Calhoun VD, Adali T, Pekar JJ, Pearlson GD. Latency (in)sensitive ICA. Group independent component analysis of fMRI data in the temporal frequency domain. Neuroimage. 2003 Nov;20(3):1661-9.

11. Hyvarinen A, Oja E. Independent component analysis: algorithms and applications. Neural Netw. 2000 May-Jun;13(4-5):411-30.

12. Mandeville JB, Marota JJ, Kosofsky BE, Keltner JR, Weissleder R, Rosen BR, et al. Dynamic functional imaging of relative cerebral blood volume during rat forepaw stimulation. Magn Reson Med. 1998 Apr;39(4):615-24.

13. Kuji I, Matsuda H, Sumiya H, Taki J, Tsuji S, Kinuya K, et al. Discrepancy between blood flow and muscarinic receptor distribution in rat brain after middle cerebral artery occlusion. Eur J Nucl Med. 1997 Jun;24(6):665-9. 

Summary

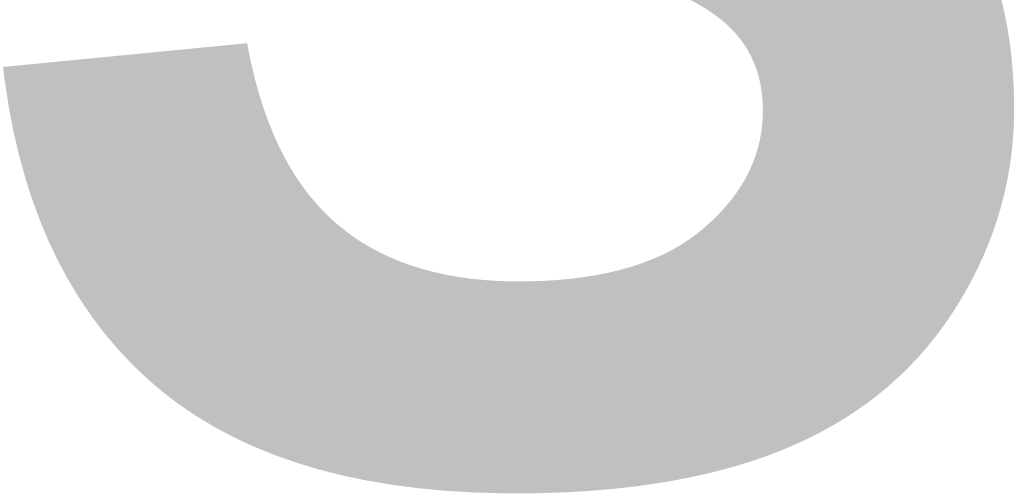


Cognitive impairment in stroke patients is thought to result from structural brain damage to both grey and white matter, leading to functional disconnection of neuronal networks. This so-called 'vascular cognitive impairment' has been associated with dysfunction of the central cholinergic system. As described in chapter 1, the present thesis explored the putative relationship between the development of cognitive impairment and damage to the central cholinergic system in an animal model of cortical cerebral infarction.

Of the numerous experimental models available to study the effects of brain ischemia, the photothrombotic stroke model is able to produce highly reproducible localized infarction of the cerebral cortex in the rat. It involves intravenous administration of a photosensitive dye followed by irradiation of a particular brain area with a focused light beam of a specific wavelength. The resulting lipid peroxidation of vascular endothelial membranes and platelets causes the formation of intravascular aggregates. Chapter 2 describes an in vivo study extending the view of the pathophysiological processes involved in the photothrombotic stroke model in the rat. It was found that vascular leakage plays a major role in the process of brain ischemia that ultimately results in focal infarction of the cerebral cortex. And probably more so than the direct occlusive thrombosis of the cortical microcirculation, as was described originally.

One of the major objectives of this thesis was to establish an experimental equivalent of human vascular cognitive impairment using the photothrombotic stroke model in rats. The neuropsychological profile of human vascular cognitive impairment is typically characterized by mental slowing: a general disturbance in the speed of information processing is observed in up to $70 \%$ of stroke patients. Both in humans and lower vertebrates like the rat, the speed of information processing can be assessed by testing reaction time performance. Behavioural tasks measuring reaction time performance are widely employed in experimental psychology, and reaction time is considered a basic element of information processing that is highly comparable between rats and humans. In chapter 3 , rats were trained in a Skinner box to master a reaction time paradigm. The type of reaction time testing we employed is similar to what is done in humans, and the nature of responding of rats in this task shows many similarities with that of humans. Following a random time interval, an auditory stimulus was presented. A high tone required pressing the left lever to obtain a food reward, whereas a low tone required pressing the right lever. The main behavioural outcomes in this behavioural task are: (1) reaction time i.e. retraction of the head from a hinged panel, and (2) motor time, being the latency between panel release and lever press. After unilateral frontal cortex infarction, reaction time performance was shown to be differentially affected between left versus right-hemisphere lesioned 
animals. Apart from a general reaction time increase of about $10 \%$ in both lesion groups, a lateralized deficit was present only after right frontal infarction as a reaction time increase of $10-15 \%$ in trials directed towards the contralesional side. These two types of reaction time deficits can be explained differently. The former resembles a gradual and general decrease in the speed of information processing that matches well with the mental slowing observed in stroke patients. The latter shows a specific impairment to initiate a contralateral motor response. Such an inability to respond to the contralesional hemispace occurs frequently in stroke patients, specifically in right hemisphere strokes, and is known as a neglect phenomenon. Therefore, reaction time performance after frontal cortical infarction in rats may be a useful tool to study particular human cognitive impairments observed in stroke patients.

Apart from deficits in information processing speed, the neuropsychological profile of vascular cognitive impairment also includes impairments in attention performance that is typically seen in patients with right hemisphere strokes. To this end, the effects of right-hemisphere photothrombotic infarction to either the frontal cortex or the parietal cortex in a behavioural paradigm measuring spatial visual attention were studied in chapter 4 . In this behavioural task, a light stimulus was presented either on the left side or on the right side of a food reward tray. One second after the light stimulus was extinguished, two levers were inserted simultaneously into the Skinner box. When the rat hit the lever on the side of the prior light stimulus (correct response), the rat was rewarded with a food pellet. When the rat hit the lever on the opposite side of the previous light stimulus (incorrect response), the rat was not rewarded. When the rat did not hit either lever within 3 seconds (omission), the rat was not rewarded. Our results showed a transient response bias immediately after frontal infarction, with a decrease in the percentage of correct responses contralateral to the lesion and an increase in contralesional omissions. Parietal infarction also resulted in a decrease in correct responses and an increase in omissions, albeit without a difference in side responding. Right frontal infarction thus induces a transient impairment in contralesional spatial visual attention that we explain as left-sided neglect, whereas right parietal infarction shows non-lateralized deficits in spatial visual attention, suggestive of global attentional impairment. This confirms the role of the right hemisphere's frontal and parietal cortices in attention performance.

Another major objective of this thesis was to characterize structural and functional changes to the central cholinergic system after photothrombotic cortical infarction in rat brain. Among other modulatory functions, the central cholinergic system is known to modulate attention performance. Therefore, the effect of cholinergic receptor blockade with scopolamine was studied in our behavioural paradigm measuring spatial visual attention. Systemic administration of scopolamine resulted in a de- 
crease in correct responses and an increase in omissions, without a difference in side responding. Cholinergic blockade thus showed non-lateralized deficits in spatial visual attention, suggestive of global attentional impairment. As such, the results described in chapter 4 underscore the well-known involvement of the cholinergic system in cognitive performance.

Next, a method was sought that would enable whole-brain assessment of the cholinergic system in a living animal. Functional imaging of this neurotransmitter system may provide novel opportunities in the diagnosis and evaluation of cognitive disorders. A number of novel ways of using functional magnetic resonance imaging (fMRI) to visualise the action of drugs on animal and human brain are becoming established tools in translational psychopharmacology. Chapter 5 describes the feasibility of pharmacological magnetic resonance imaging (phMRI) as a means to detect cholinergic muscarinic receptor activation in rat brain. Blood oxygenation leveldependent (BOLD) MRI and contrast-enhanced cerebral blood volume (CBV)weighted MRI were conducted during pharmacological stimulation with pilocarpine, a non-selective muscarinic acetylcholine receptor agonist. BOLD and CBV responses were assessed in drug-naïve rats, and in rats pretreated with methyl-scopolamine in order to block peripheral muscarinic effects. Both in drug-naïve and methylscopolamine pretreated animals, pilocarpine induced significant BOLD signal increases primarily in the cerebral cortex. With contrast-enhanced CBV-weighted MRI, positive CBV responses were detected in the cerebral cortex, thalamus, and hippocampus whereas a negative CBV response was observed in the corpus striatum. It was concluded that pilocarpine induced significant activation responses in brain regions that are known to have a high density of muscarinic receptors. These experiments demonstrate that phMRI of muscarinic receptor stimulation in rats allows functional assessment of the cholinergic system in vivo. Subsequently, this neuroimaging method was applied to measure cholinergic muscarinic receptor activation after brain infarction, as is described in the appendix. It was hypothesized that subcortical ischemia would result in the degeneration of cholinergic projections toward the cerebral cortex, and thereby reduced cortical muscarinic receptor activation. Transient middle cerebral artery occlusion was performed to induce subcortical ischemia in a small number of rats, followed after two weeks by phMRI of muscarinic receptor activation. The results of this pilot study suggest that direct subcortical ischemic damage leads to diminished striatal muscarinic receptor activation as well as diminished muscarinic receptor stimulation in the cerebral cortex. However, further experiments are required to establish these preliminary findings.

Chapter 6 reports on remote effects of photothrombotic infarction to the frontal cortex on muscarinic receptor activation as well as cholinergic cell number and vol- 
ume in several basal forebrain nuclei. First, phMRI assessing cerebral muscarinic receptor activation was performed at 1 and 3 weeks after left or right frontal cortex infarction. We found near-significant reductions in muscarinic receptor activation inside the ischemic area after right-sided cortical infarction. Moreover, significant activation changes at 3 weeks after right-hemisphere infarction were found in the motor cortex adjacent to the lesion, and in the sensory cortex as well as striatum distant from the lesion. Second, stereological assessment of choline acetyltransferase (ChAT)-immunopositive cells was conducted in several basal forebrain nuclei. No significant alterations were found in cholinergic cell number and volume following focal infarction to the right of left frontal cortex. Since cholinergic projections do not seem to degenerate after focal cortical infarction, functional imaging of muscarinic receptor activation may provide a sensitive tool for the evaluation of therapy in vascular cognitive impairment.

Finally, in chapter 7 the results of the conducted studies are discussed and recommendations for future research are made. 


\section{Samenvatting}

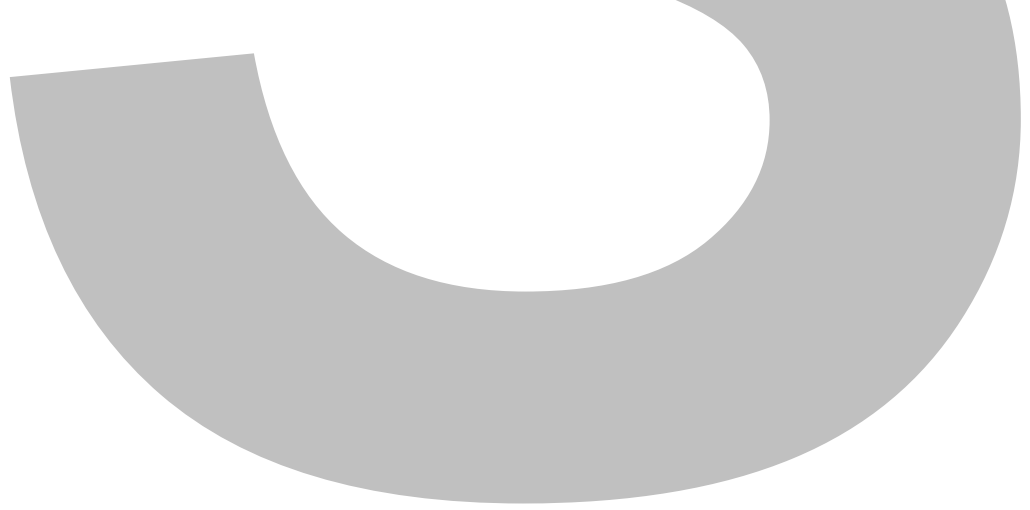


Cognitieve stoornissen bij patiënten met een beroerte worden verondersteld het resultaat te zijn van structurele hersenschade van zowel grijze als witte stof, leidend tot functionele disconnectie van neuronale netwerken. Deze zogenaamde 'vasculaire cognitieve beperking' is geassocieerd met disfunctie van het centrale cholinerge systeem. Zoals beschreven in hoofdstuk 1, richt dit proefschrift zich op de veronderstelde relatie tussen het ontstaan van cognitieve stoornissen en beschadiging van het centrale cholinerge systeem in een diermodel voor het herseninfarct.

Van de vele diermodellen die beschikbaar zijn voor het bestuderen van de effecten van hersenischemie, is het met behulp van het fototrombotisch model mogelijk zeer reproduceerbare en gelokaliseerde infarcten in de hersenschors bij de rat te verkrijgen. Hierbij wordt een lichtgevoelige kleurstof intraveneus ingespoten waarna door de schedel heen een hersengebied wordt beschenen met een lichtstraal van specifieke golflengte (laser). Dit leidt via lipidperoxidatie van vaatendotheel en bloedplaatjes tot vorming van aggregaten en dientengevolge microvasculaire trombose. Hoofdstuk 2 beschrijft een studie die de kijk op de pathofysiologische processen betrokken bij fototrombotisch model voor herseninfarcering verbreedt. Vaatlekkage blijkt een belangrijke rol te spelen in het ontstaan van een herseninfarct. En waarschijnlijk zelfs meer dan de microvasculaire trombose zoals die oorspronkelijk werd beschreven.

Een van de voornaamste doelen van dit proefschrift was het ontwikkelen van een experimenteel equivalent van vasculaire cognitieve stoornissen in mensen, gebruikmakend van het fototrombotisch beroertemodel in ratten. Het neuropsychologische profiel van vasculaire cognitieve beperking wordt vooral gekenmerkt door mentale traagheid: verstoring van de snelheid van informatieverwerking wordt gezien bij ongeveer $70 \%$ van de patiënten met een beroerte. Zowel bij mensen als lagere gewervelde dieren zoals de rat, kan de snelheid van informatieverwerking worden bepaald door testen van het reactievermogen. Gedragstaken die de reactietijd meten, worden breed toegepast in de experimentele psychologie. Reactiesnelheid wordt gezien als de basis van informatieverwerking, en is gebleken vergelijkbaar te zijn tussen ratten en mensen.

In hoofdstuk 3 werden ratten in een Skinner box getraind om een reactietijdtaak te beheersen. Het type reactietest dat werd gebruikt is gelijk aan dat bij mensen, en de aard van de respons bij ratten vertoont in deze taak veel gelijkenissen met die bij mensen. De taak begint met een geluidsstimulus die wordt gepresenteerd terwijl een rat met zijn hoofd tegen een paneel rust. Bij een hoge toon levert het indrukken van het pedaal links met het hoofd een voedselbeloning op, terwijl bij een lage toon het indrukken van het pedaal rechts van het hoofd nodig is voor het krijgen van een voedselbeloning. De belangrijkste uitkomstmaten in deze gedragstaak zijn: (1) reac- 
tietijd, te weten de tijd benodigd voor terugtrekken van het hoofd, en (2) motortijd, zijnde de latentie tussen terugtrekken van het hoofd en indrukken van het pedaal. Het reactievermogen bleek op verschillende manieren te zijn aangedaan bij ratten met een herseninfarct. Enerzijds was er een algemene toename in reactietijd bij ratten met een herseninfarct in de frontale hersenschors van de linker of rechter hersenhelft. Anderzijds was er een gelateraliseerd defect specifiek voor het reactievermogen naar links bij ratten met een herseninfarct in de rechter hemisfeer. Deze twee soorten stoornissen in reactiesnelheid kunnen verschillend verklaard worden. De graduele en algemene afname in de snelheid van informatieverwerking past bij de mentale vertraging zoals die gezien wordt bij beroertepatiënten. De specifieke beperking tot het initiëren van een contralaterale respons komt ook bij patiënten met een beroerte in de rechter hersenhelft vaak voor, en staat bekend als een neglect fenomeen. Hierbij zijn de zintuiglijke functies intact, maar is er desondanks een onvermogen tot het reageren op stimuli aan de zijde contralateraal van het letsel in de hersenen. Testen van het reactievermogen bij ratten met een herseninfarct van de frontale hersenschors kan hierom een bruikbaar instrument zijn voor het bestuderen van bepaalde cognitieve stoornissen zoals die voorkomen bij beroertepatiënten.

Naast tekorten in de snelheid van informatieverwerking, wordt het neuropsychologische profiel van vasculaire cognitieve beperking ook gekenmerkt door stoornissen in aandachtsfuncties, vooral bij patiënten met een beroerte in de rechter hersenhelft. Hierom werden in hoofdstuk 4 gedragseffecten gemeten na fototrombotische herseninfarcering in ofwel de frontale ofwel de parietale schors van de rechter hersenhelft. In de gebruikte aandachtstaak werd een lichtstimulus gepresenteerd aan ofwel de linkerkant ofwel de rechterkant van de rat. Vervolgens werden aan weerszijden van de rat simultaan twee pedalen in de Skinner box gebracht. Wanneer de rat het pedaal aan de kant van de voorafgaande lichtstimulus indrukte (correcte respons), werd deze beloond met voedsel. Wanneer de rat het pedaal indrukte aan de tegenovergestelde kant van de voorafgaande lichtstimulus (incorrecte respons), werd deze niet beloond. Wanneer de rat binnen 3 seconden geen van beide pedalen indrukte (omissie), werd deze ook niet beloond. In de eerste dagen na een frontaal hersenschorsinfarct werd een transiënte responsfout gezien, met een afname in het percentage van correcte responsen en een toename in het aantal omissies contralateraal van het letsel. Ook infarcering van de parietale hersenschors resulteerde in een afname van het percentage correcte responsen en een toename in het aantal omissies, doch zonder verschil tussen contralaterale en ipsilaterale responsen. Bij ratten induceert een infarct van de rechter frontale hersenschors dus een tijdelijke stoornis in ruimtelijke visuele aandacht contralateraal aan het letsel, hetgeen te verklaren is als een linkszijdig neglect fenomeen. Een infarct van de rechter parietale 
hersenschors leidt echter tot een globaal tekort in ruimtelijke visuele aandacht. Deze studie bevestigt de rol van de frontale en parietale schors van rechter hersenhelft in aandachtsfuncties.

Een ander belangrijk doel van dit proefschrift was het karakteriseren van structurele en functionele veranderingen in het centrale cholinerge systeem na fototrombotische infarcering in rattenhersenen. Het is bekend dat het centrale cholinerge systeem onder andere een modulerende rol speelt bij aandachtsfuncties. Daarom werd het effect bestudeerd van blokkade van cholinerge receptoren door scopolamine in de bovenbeschreven gedragstaak voor ruimtelijke visuele aandacht. Systemische toediening van scopolamine resulteerde in een afname van correcte responsen en een toename van omissies, zonder verschil tussen contralaterale en ipsilaterale responsen. Net als na infarcering van de rechter parietale hersenschors, leidt blokade van cholinerge receptoren dus tot een globaal tekort in ruimtelijke visuele aandacht. Zodoende onderstrepen de resultaten beschreven in hoofdstuk $\mathbf{4}$ de betrokkenheid van het cholinerge systeem bij cognitief functioneren.

Vervolgens is een methode gezocht waarmee het cholinerge systeem van de hersenen in zijn geheel zichtbaar te maken zou kunnen zijn bij een levend wezen. Functionele beeldvorming van dit neurotransmittersysteem biedt wellicht nieuwe mogelijkheden voor de diagnose en evaluatie van cognitieve stoornissen bij mensen. Hoofdstuk 5 beschrijft de haalbaarheid van farmacologische magnetische resonantie beeldvorming (phMRI) als een instrument voor het detecteren van cholinerge muscarine receptoractivatie in rattenhersenen. Blood oxygenation level-dependent (BOLD-MRI) en contrastversterkte cerebral blood volume (CBV)-gewogen MRI werden uitgevoerd gedurende toediening van pilocarpine, een niet-selectieve muscarine acetylcholine receptor agonist. BOLD- en CBV-responsen werden bepaald in ratten waarbij perifeer muscarinerge effecten geblokkeerd waren met methylscopolamine, dat de bloed-hersenbarriere niet passeert. Pilocarpine veroorzaakte een significante toename in BOLD signaal, voornamelijk in de cerebrale cortex. Met contrastversterkte CBV-gewogen MRI werden positieve CBV responsen gevonden in de cerebrale cortex, thalamus en hippocampus; een negatieve CBV-respons werd geobserveerd in het striatum. Toediening van pilocarpine leidt derhalve tot veranderingen in hersengebieden die een hoge dichtheid van muscarine receptoren kennen. Deze experimenten tonen aan dat phMRI met muscarine receptor stimulatie in ratten een functionele beoordeling van het cholinerge systeem in vivo mogelijk maakt. Vervolgens werd deze beeldvormende techniek gebruikt voor het detecteren van cholinerge muscarine receptor activatie na herseninfarcering (appendix). De hypothese was dat subcorticale ischemie zou resulteren in degeneratie van cholinerge projecties naar de cerebrale cortex, en zodoende zou leiden tot een afname in corticale muscarine 
receptor activatie. Subcorticale ischemie werd geïnduceerd door transiënte occlusie van de arteria cerebri media, en na twee weken gevolgd door phMRI van muscarine receptor activatie. De resultaten van deze pilot studie suggereren dat subcorticale ischemische schade leidt tot verlaagde muscarine receptor activatie in zowel het striatum als de cerebrale cortex. Aanvullende experimenten zijn echter nodig voor het bevestigen van deze voorlopige resultaten.

Hoofdstuk 6 rapporteert de effecten van fototrombotische infarcering van de frontale hersenschors op zowel muscarine receptor activatie als het aantal en volume van cholinerge cellen in verscheidene kernen in de basale voorhersenen. Ten eerste werd phMRI toegepast voor het beoordelen van cerebrale muscarine receptor activatie 1 en 3 weken na infarcering van de linker danwel rechter frontale hersenschors. Er werd een afname van muscarine receptor activatie gevonden binnen het ischemisch gebied na rechtszijdige corticale infarcering. Daarenboven werden 3 weken na infarcering van de rechter frontale hersenschors significante activatieveranderingen gevonden op afstand van het ischemisch gebied, te weten in de motore schors grenzend aan het ischemisch letsel en in de sensore cortex op grotere afstand. Ten tweede werd miscroscopische beoordeling van cholinerge acetyltransferase (ChAT)immunopositieve cellen uitgevoerd in verscheidene kernen in de basale voorhersenen. Er werden geen significante veranderingen gevonden in het aantal en volume cholinerge cellen na infarcering van de rechter danwel linker frontale hersenschors. Omdat cholinerge projecties niet structureel lijken te degenereren na infarcering van de hersenschors, kan functionele beeldvorming van muscarine receptor activatie een gevoelig instrument zijn voor de evaluatie van therapie bij vasculaire cognitieve beperking.

Ten slotte worden in hoofdstuk 7 de resultaten van de uitgevoerde studies bediscussieerd en aanbevelingen voor toekomstig onderzoek gedaan. 



\section{Dankwoord}

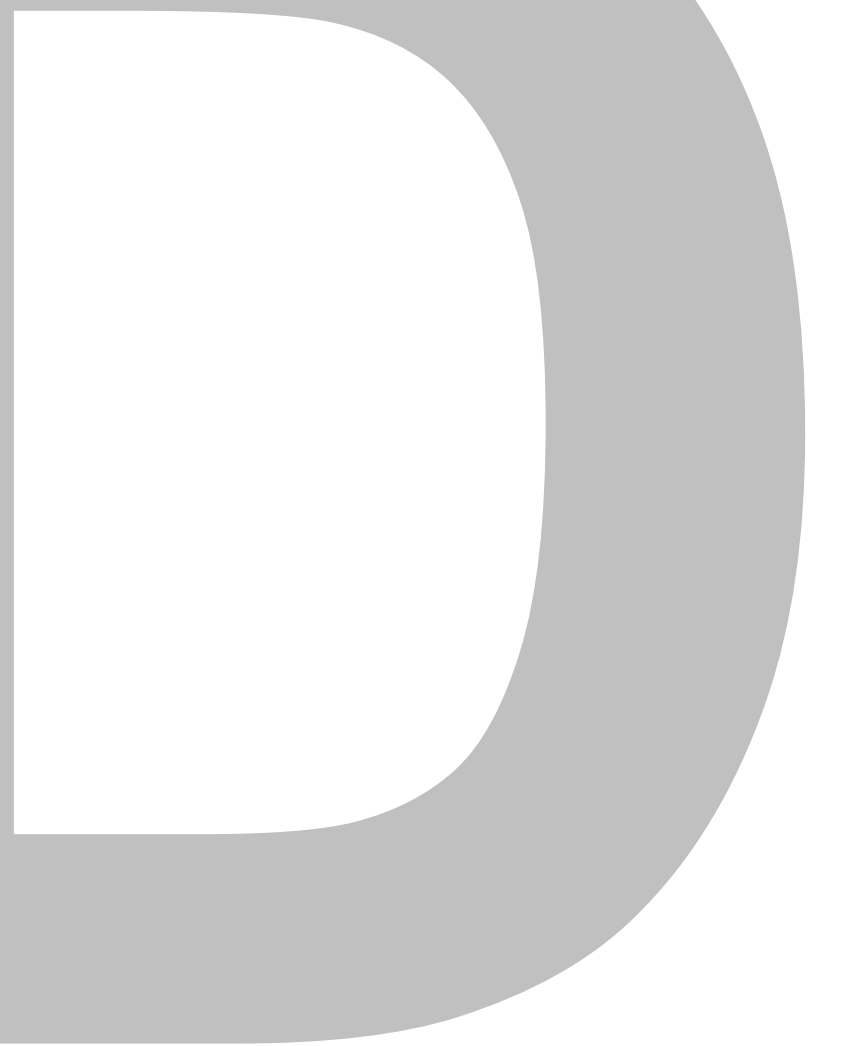


Dit is wat ik altijd al heb willen doen: eigen onderzoek. Ooit kwam de promovendus na jarenlang vorsen met een "Eureka!" van zolder om zijn ontdekkingen aan de wereld te presenteren. Tegenwoordig dienen de resultaten van veelal vooropgezet onderzoek binnen een viertal jaren op de plank te liggen. Ik ben blij dat in de huidige tijd, het in dit proefschrift beschreven onderzoek zijn eigen koers heeft kunnen varen. Graag zou ik langs deze weg de mensen die hieraan hebben bijgedragen willen bedanken voor het geduld, vertrouwen en het wijzen van de weg.

Op weg. Harry Steinbusch, bedankt voor alles wat je voor mij en mijn onderzoek hebt gedaan. Onder jouw leiding biedt het laboratorium Basale Neurowetenschappen een stimulerende en internationale smeltkroes waar ideeën vrijelijk kunnen worden ontwikkeld. Jaap Troost, jij schiep de mogelijkheid om vanuit ziekenhuis naar laboratorium en weer terug te komen. Door de loop der dingen was promotorschap niet meer mogelijk, maar ik ben blij dat je tot het eind toe een rol bent blijven spelen. Martien Limburg, jij faciliteerde wanneer nodig het afronden van dit onderzoek tijdens mijn opleiding tot neuroloog. Robert van Oostenbrugge, we gingen in het begin letterlijk de boot in en figuurlijk bijna. Mede door jouw manier van begeleiden is dit onderzoek komen bovendrijven. Die manier waardeer ik enorm en ligt mij erg goed: open, kritisch en geduldig. Rick Dijkhuizen, wat als spin-off begon is uitgegroeid tot de ongeveer de helft van dit proefschrift. Haast belangeloos stond jij altijd met raad en daad klaar. Je oprechtheid, enthousiasme en oprecht enthousiaste reactie toen ik je vroeg of je compromotor wilde zijn, sieren je.

Het ruime sop. Het laboratorium Basale Neurowetenschappen is waar de trossen werden los gegooid. Ik dank Wiel Honig en Richard Frijnts voor het leren omgaan met proefdieren en de nodige assistentie bij opzet en uitvoer van de fototrombose. Er volgde een kort intermezzo op het microcirculatie laboratorium van de afdeling Fysiologie, waarbij de begeleiding van Mirjam oude Egbrink en Viviane Heijnen onmisbaar waren. Arjan Blokland, jij hebt mijn aanvankelijke scepsis met betrekking tot gedragsonderzoek bij ratten gelogenstraft. Dank voor de prettige samenwerking. Kris Rutten en Marjolein Liedenbaum, jullie geweldige inzet en resultaten vormden de basis voor wat uiteindelijk dit proefschrift is geworden. Voor het bijbrengen van de mores van het lab, leren werken met microscoop en supervisie bij kleuringen is de twee-eenheid Hellen Steinbusch en Marjan Markerink van onnoembaar belang geweest. Anouk Roemen, jij optimaliseerde het kleuringsprotocol voor de volumemetingen van het infarct. Lillian Garrett, thanks so much in joining me for countless hours of stoic cell counting. Er is nu echt een eind gekomen aan een periode van mannelijke AiO's, onderzoeksmannen van het eerste uur: Ronald Deumens, Guido Koop- 
mans, Daniel van den Hove, Bart Rutten en Yasin Temel. Gezelligheid kent geen tijd, wel mooie herinneringen. Sitting on a sack of beans, sitting down in New Orleans, you wouldn't believe what I've seen (Apache Rose Peacock, Red Hot Chili Peppers).

De Cycloop. Halverwege werd aangemeerd bij het laboratorium In Vivo NMR te Utrecht. Gerard van Vliet en Annette van der Toorn dank ik voor hun stille kracht. Zonder jullie kan er geen scan worden gemaakt. Ona Wu, you introduced me into the world of MR data processing and analysis, which regularly flabbergasted me. Jet van der Zijden, sommige van jouw ogenschijnlijk mislukte experimenten kwamen mij zeer goed van pas. Raoul Oude Engberink, jij bood me geregeld port en een slaapplaats en ik bewaar zeer goede herinneringen aan onze samenwerking. Wim Otte en Kajo van der Marel, jullie bereidwilligheid om op de meest onverwachte momenten de data aan weer een hernieuwde analyse te onderwerpen is indrukwekkend. Nog een laatste list en dan kan komen we wel weg. Er is licht aan het eind van het boorgat.

Een aparte vermelding verdient studievriend Remco Knol. Ik kan je altijd bellen voor een illustratie of figuur. Er zullen ongetwijfeld nog verzoeken volgen. Dank in ieder geval voor het dorsaal aanzicht van de rattenhersenen (cover, hoofdstukken 3 en 4). Ook dank ik Stefan Dieleman voor hulp bij de offline bloeddrukmetingen (hoofdstuk 5). And Guerbet Group for kindly providing Sinerem ${ }^{\circledR}$ (hoofdstukken 5, 6, Appendix).

Mijn paranimfen. Govert Hoogland, wij deelden jarenlang een kamer en sindsdien steeds meer van het leven. Vaderschap, sport, theater. Daarnaast is er veel wat een dokter van een doctor kan leren. Jeroen Trip, ook onze levens kruisen elkaar de laatste jaren steeds meer, zowel in het werk als daarbuiten. Er valt nog genoeg uit te kristalliseren. Ik ben trots dat jullie naast mij willen staan tijdens de verdediging van dit proefschrift.

Mijn ouders. Renne van Ruth, Henk Hoff en Aaltje de Vries. Jullie hebben me ongedwongen gevormd en altijd gesteund. Dankzij jullie voel ik me overal thuis.

Mijn Sirenen. Danitsja, lief, wat moet ik zonder jou beginnen? Jij hebt niet alleen dit proefschrift vorm gegeven, maar mijn hele leven. Samen met Lenore, onze lieve kleine meid. Ik luister zo graag naar jullie lied. Nu zal daar meer tijd voor zijn. Haal de was uit mijn oren en snij me los. 


\section{Curriculum vitae}


Erik Inne Hoff was born in Amsterdam on January 17th 1975. He grew up in the villages of Santpoort and Hoofddorp. In 1993, he graduated from pre-university eduction at Hageveld College in Heemstede and started studying medicine at the University of Amsterdam (UVA). He received his Masters degree in 1997 and took Hippocrates' oath in 2000. Erik left Amsterdam the same year, to start as a non-resident in Neurology at the university hospital Maastricht. The research presented in this thesis was conducted at the School for Mental Health and Neuroscience Maastricht and the Image Sciences Institute Utrecht. Since 2004, Erik has resumed his clinical occupation as a resident in Neurology at the university hospital Maastricht.

Erik Inne Hoff werd geboren in Amsterdam op 17 januari 1975. Hij groeide op in de dorpen Santpoort en Hoofddorp. In 1993 ontving hij zijn VWO-diploma aan het College Hageveld te Heemstede en begon de studie Geneeskunde aan de Universiteit van Amsterdam (UvA). Hij ontving zijn doctoraaldiploma in 1997 en legde de eed van Hippocrates af in 2000. In datzelfde jaar verliet Erik Amsterdam, om te beginnen als AGNIO Neurologie in het academisch ziekenhuis Maastricht. Het onderzoek beschreven in dit proefschrift werd uitgevoerd aan de School for Mental Health and Neuroscience te Maastricht en het Image Sciences Institute te Utrecht. In 2004 hervatte Erik zijn klinische werkzaamheden als AIOS Neurologie in het academisch ziekenhuis Maastricht. 

Publications

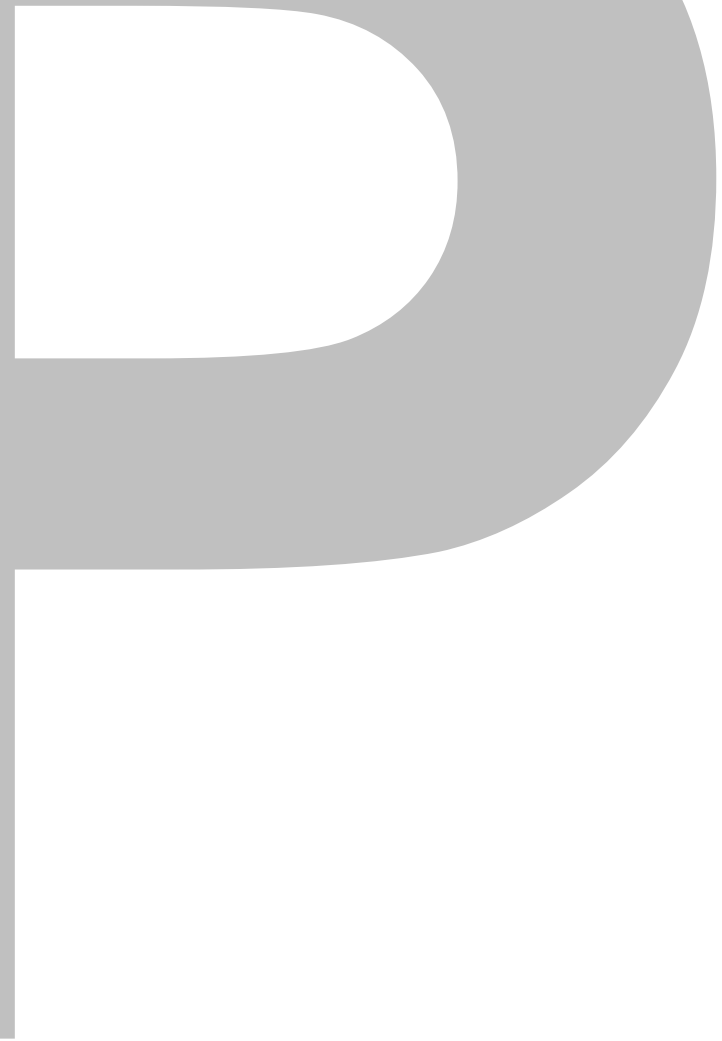




\section{Publications}

Hoff El, Steinbusch HW, van Oostenbrugge RJ, Garrett L, Otte W, Van der Marel K, Dijkhuizen RM. Alterations in the cholinergic system after frontal cortex infarction in rat brain: pharmacological magnetic resonance imaging of muscarinic receptor activation and stereological analysis of cholinergic basal forebrain neurons (submitted)

Hoff El, van Oostenbrugge RJ, Otte W, Wu O, Steinbusch HW, Dijkhuizen RM. Pharmacological magnetic resonance imaging of muscarinic acetylcholine receptor activation in rat brain. (submitted)

Oude Engberink RD, Blezer EL, Hoff El, van der Pol SM, van der Toorn A, Dijkhuizen $\mathrm{RM}$, de Vries HE. MRI of monocyte infiltration in an animal model of neuroinflammation using SPIO-labeled monocytes or free USPIO. J Cereb Blood Flow Metab. 2008;28:841-51.

Frederix K, Chauhan AK, Kisucka J, Zhao BQ, Hoff El, Spronk HM, Ten Cate H, Wagner DD. Platelet adhesion receptors do not modulate infarct volume after a photochemically induced stroke in mice. Brain Res. 2007;1185:239-45.

Hoff El, van Oostenbrugge RJ, Liedenbaum M, Steinbusch HW, Blokland A. Effects of right-hemisphere cortical infarction and muscarinic acetylcholine receptor blockade on spatial visual attention performance in rats. Behav Brain Res. 2007;178:62-9.

Hoff El, Blokland A, Rutten K, Steinbusch HW, van Oostenbrugge RJ. Dissociable effects in reaction time performance after unilateral cerebral infarction: a comparison between the left and right frontal cortices in rats. Brain Res. 2006;1069:182-9.

Hoff El, oude Egbrink MG, Heijnen VV, Steinbusch HW, van Oostenbrugge RJ. In vivo visualization of vascular leakage in photochemically induced cortical infarction. J Neurosci Methods. 2005;141:135-41

Hoff El, Kruys EM, Voogd E. Pijn in de nek op nieuwjaarsdag. Ned Tijdschr Geneeskd Stud ed 2000;1:14-7 